\title{
A Valuation Framework for Informing Grid Modernization Decisions: Guidelines
}

\section{Guidelines on the principles and process of valuing grid services and technologies}

\section{March 2019}

Grid Modernization Laboratory Consortium

National Association of Regulatory Utility Commissioners 


\section{DOCUMENT AVAILABILITY}

Reports produced after January 1, 1996, are generally available free via US Department of Energy (DOE) SciTech Connect.

Website www.osti.gov

Reports produced before January 1, 1996, may be purchased by members of the public from the following source:

National Technical Information Service

5285 Port Royal Road

Springfield, VA 22161

Telephone 703-605-6000 (1-800-553-6847)

TDD 703-487-4639

Fax 703-605-6900

E-mail info@ntis.gov

Website http://classic.ntis.gov/

Reports are available to DOE employees, DOE contractors, Energy Technology Data Exchange representatives, and International Nuclear Information System representatives from the following source:

Office of Scientific and Technical Information

PO Box 62

Oak Ridge, TN 37831

Telephone 865-576-8401

Fax 865-576-5728

E-mail reports@osti.gov

Website http://www.osti.gov/contact.html

This report was prepared as an account of work sponsored by an agency of the United States Government. Neither the United States Government nor any agency thereof, nor any of their employees, makes any warranty, express or implied, or assumes any legal liability or responsibility for the accuracy, completeness, or usefulness of any information, apparatus, product, or process disclosed, or represents that its use would not infringe privately owned rights. Reference herein to any specific commercial product, process, or service by trade name, trademark, manufacturer, or otherwise, does not necessarily constitute or imply its endorsement, recommendation, or favoring by the United States Government or any agency thereof. The views and opinions of authors expressed herein do not necessarily state or reflect those of the United States Government or any agency thereof. 


\section{A Valuation Framework for Informing Grid Modernization Decisions: Guidelines}

A summary of the principles and process for valuing grid services and technologies

Grid Modernization Laboratory Consortium Members:

L. C. Markel ${ }^{8}$

A. Cooke ${ }^{2}$

S. W. Hadley ${ }^{1,8}$

A. D. Mills ${ }^{5}$

P. W. O'Connor ${ }^{1}$

V. N. Vargas ${ }^{6}$

A. K. Wolfe ${ }^{1}$

V. Koritarov ${ }^{3}$

C. A. Goldman ${ }^{5}$

M. Kintner-Meyer ${ }^{2}$

P. Gagnon ${ }^{4}$

M. Ruth ${ }^{4}$

A. Somani ${ }^{2}$

G. Porro ${ }^{4}$

R. F. Jeffers ${ }^{6}$

In partnership with the National Association of Regulatory Utility Commissioners
J. Brinch ${ }^{7}$
K. Worthington ${ }^{7-}$
D. Byrnett ${ }^{7}$

${ }^{1}$ Oak Ridge National Laboratory

${ }^{2}$ Pacific Northwest National Laboratory

${ }^{3}$ Argonne National Laboratory

${ }^{4}$ National Renewable Energy Laboratory

${ }^{5}$ Lawrence Berkeley National Laboratory

${ }^{6}$ Sandia National Laboratories

${ }^{7}$ National Association of Regulatory Utility Commissioners

${ }^{8}$ SRA International Inc., a General Dynamics Information Technology company

March 2019 



\section{Executive Summary A Valuation Framework for Informing Grid Modernization Decisions}

Advances in technologies to provide electric power (e.g., renewable energy and battery storage), measurements and monitoring (e.g., synchrophasors, smart meters), communication (e.g., cellular service, Internet utilities), computing (powerful distributed microprocessors), and innovations in grid topologies and configurations (e.g., microgrids) have markedly changed the options available to provide electricity to end-users. The value propositions for electrical service have similarly become much more complex and diverse. The values of a grid design option can no longer be expressed by just using historical costemissions-reliability metrics. Nor can major decisions be made through closed analysis without consideration of the different stakeholders affected by a decision. This valuation framework provides a structure for valuation that is deliberate, inclusive, and transparent, documenting the intermediate and final results to ensure clarity, accountability and repeatability.

The expanded nature of the available grid options, the multiple criteria for making a decision, and the diversity of stakeholders affected by the decision require a more robust, transparent and inclusive valuation process for choosing among alternatives. Unfortunately, across the electric power sector, there are few "best practices" for designing methodologies to assess and compare the value of diverse courses of action. Alternatives to be considered range from investing in generation, transmission, or distribution assets, to compensation mechanisms for distributed technologies, to options for energy consumers to actively vary their energy purchases from the grid in response to grid status and pricing.

Investment decisions and policy options are reviewed and interpreted by multiple stakeholders with different motivations and interests. The resulting value assessments often vary widely for the same or similar types of projects. ${ }^{1}$ These differences can be attributed to different assumptions with respect to economic and engineering inputs; time, geographic, and power system scales; the closed-source inner workings of modeling tools and analysis methodologies employed for the valuation; stakeholder-specific choices of the key metrics or grid attributes that inform the valuation; and/or the choice of which grid investment, design, or operation alternatives to compare. The objective of this project is to develop a framework to provide guidance for designing clear, transparent, and repeatable valuation methodologies, and to develop practices to generate results that are easier to interpret and easier to compare.

The concept of "value" in this context goes far beyond monetary savings and costs. Value encompasses many elements, including reliability, resiliency, sustainability, security, environmental quality, public health and safety, and economic impacts. Metrics to characterize value may be quantitative or qualitative; often, defining those metrics is not straightforward and may in fact be controversial. It is important to be explicit about how all metrics are defined, are gauged, and contribute to the valuation. The proposed valuation framework described in this Guidelines Document delineates a process for doing so.
The valuation framework provides a deliberate methodology to identify the criteria for deciding among alternative grid strategies or configurations; determining what information is required to inform that decision; selecting the methods and tools that can best provide that information within time and budget constraints; and interpreting the results to compare multi-criteria valuation assessments.

The framework defines 11 activities within 4 phases, as shown in Figure ES-1:

\footnotetext{
${ }^{1}$ E.g., Trabish, Herman K., "How two value-of-solar studies add up to no clear value of solar: Is rooftop solar really worth 90\% less in Montana than in Maryland?" Utilitydive.com, May 10, 2018, https://www.utilitydive.com/news/how-two-value-of-solar-studies-add-up-to-no-clear-value-of-solar/522892/
} 
A. Specify the purpose, scope and goal of the study and identify stakeholders;

B. Define decision criteria and information needs;

C. Design the analysis procedures, tools and models to provide necessary information;

D. Perform analyses and interpret results.

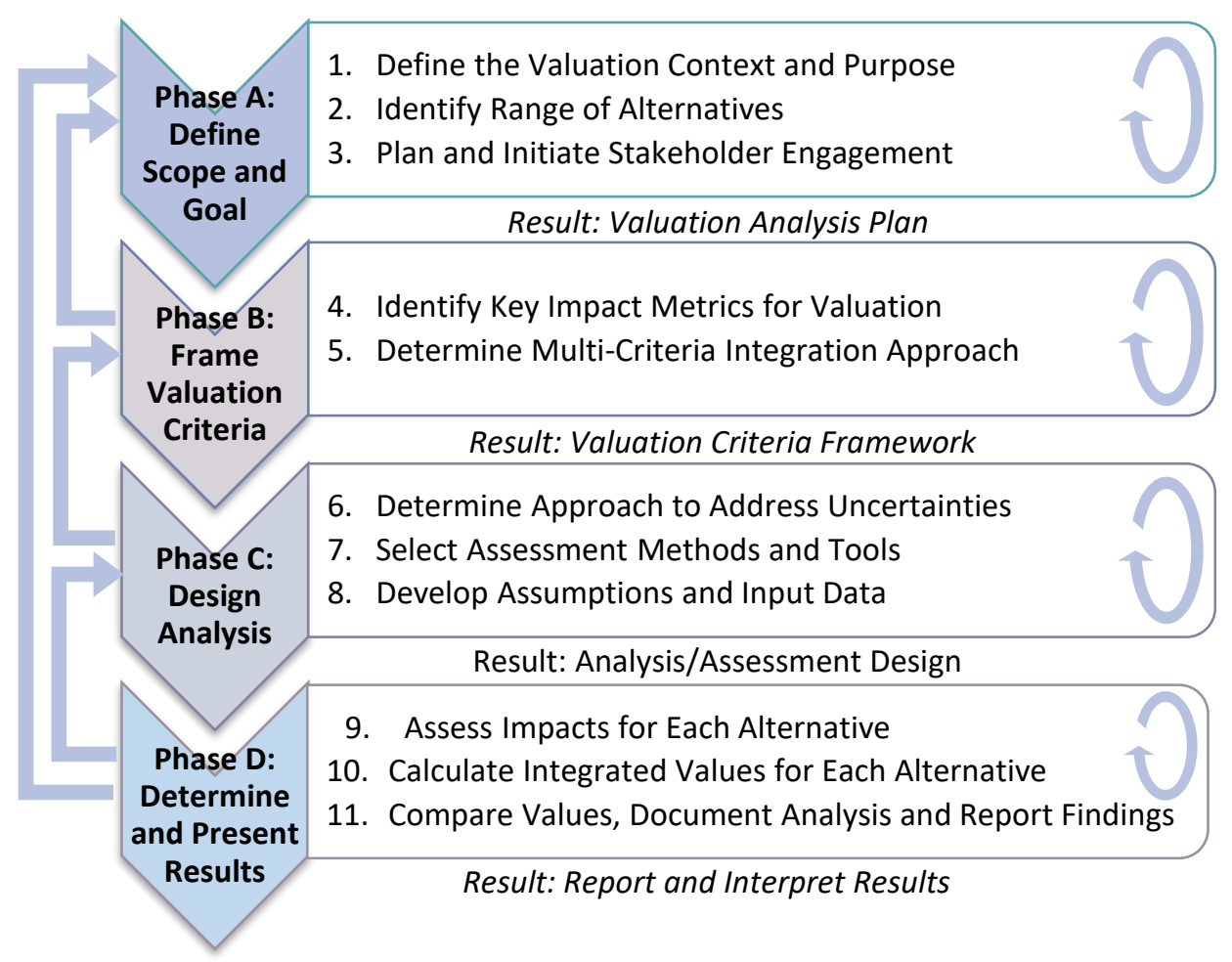

Figure ES-1. Valuation framework process.

This Guidelines Document describes the valuation framework and each of its activities. It also provides some reference materials and suggested practices to assist the user in designing a methodology that addresses a specific question or choice.

As there are many valid ways to perform a valuation study, the framework is not meant to be a prescriptive "how to" manual or an official rulebook for valuation. Rather, it describes a process consisting of activities that should be part of a valuation that is comprehensive, transparent, and soundly based on technical and economic principles.

It starts with a desire for information. In most circumstances this information is needed for a decision: choosing among alternatives for grid modernization, expansion, design, operating procedures, and so on. To make such a choice, the decision makers need to compare the values of each alternative, and value is a multifaceted and often subjective attribute. The valuation framework provides a structure to identify what information decision makers need to make a good decision; how to obtain this information; and how to evaluate it. By being systematic in determining the information needs and the impact analyses required to make a sound decision, and being mindful of the choices made in designing the analysis methodsincluding assumptions, approximations, and trade-offs - the valuation framework presents a process that increases the likelihood that the ultimate decision (i.e., choice of alternative) is sound and is acceptable to affected stakeholders. This document offers a valuation framework to decision makers and analysts, with guidance for applying and adapting it to address their specific questions. 


\section{Acknowledgments}

The Lab Team and Stakeholder Advisory Group would like to express a deep appreciation to the late Jeannette "Jan" Brinch of the National Association of Regulatory Utility Commissioners (NARUC) for her experience, tenacity, and collaborative spirit throughout the 3 years of this project. Jan's contributions were essential to the project's accomplishments.

The Lab Team wants to thank our Stakeholder Advisory Group members who provided guidance and support through attendance at webinars and multi-day seminars; helpful comments on draft papers; encouragement on the framework; and insights into what those involved in the development, operation, and regulation of the grid system outside of the national laboratory system know about the issues and concerns regarding grid modernization valuation. Their expertise and wealth of knowledge were instrumental in shaping and directing the project. Kerry Worthington, Danielle Sass Byrnett and Dominic Liberatore of the National Association of Regulatory Utility Commissioners provided coordination between the Stakeholder Advisory Group and the Lab Team.

\section{Grid Valuation Framework Stakeholder Advisory Group (past and present)}

Michael Bailey, Senior Engineer on System Adequacy, Western Electricity Coordinating Council Denis Bergeron, Director of Energy Program, Maine Public Utilities Commission Betsy Beck, Director, Transmission Policy, American Wind Energy Association Gary Brinkworth, Director of Technology Innovation, Tennessee Valley Authority (TVA)

Lilian Bruce, Strategic Research, Electric Power Board, Chattanooga

John Buechler, Director, Eastern Interconnection Planning Council

Erin Erben, Rates and Financial Planning Manager, Tacoma Power, and EPRI (former)

Ed Finley, Chairman - North Carolina Utilities Commission; Alternate: Kim Jones

Ben Hobbs, Director, Environment, Energy, Sustainability \& Health Institute, Johns Hopkins University

David Kolata, Executive Director, Citizens Utility Board

Ron Lehr, Consultant - Western Clean Energy Advocates, Western Grid Group

Jonathan Lesser, Continental Economics Inc.

Enrique Mejorada, Director of Energy Policy Modeling and Analysis, Pacific Gas \& Electric

Rohan Ma, Solar City Corporation (former); Alternate: Ryan Hanley (former)

Eliah Gilfenbaum, Tesla

Michal Moore, Earth-Energy Systems Integrated Graduate Education and Research Training (IGERT), Cornell University

Jeff Morris, Representative, Washington State Legislature

Bernard Neenan, EPRI (retired), Energy Resource Economics (current)

Ray Palmer, Federal Energy Regulatory Commission (FERC) (former)

Jeffery Roark, Power Delivery \& Utilization, Electric Power Research Institute (EPRI)

Matthew Shuerger, Commissioner, Minnesota PUC

Sekou Sidime, Commonwealth Edison

Tom Sloan, Kansas State House of Representatives (former)

J. T. Smith, Director, Policy Studies, Midcontinent ISO

Nick Wagner, Commissioner, Iowa PUC

David Whiteley, Director, Eastern Interconnection Planning Council (former)

This research was supported by the Grid Modernization Initiative of the US Department of Energy (DOE) as part of its Grid Modernization Laboratory Consortium, a strategic partnership between DOE and the national laboratories to bring together leading experts, technologies, and resources to collaborate on the goal of modernizing the nation's grid. We would like to thank all of our DOE sponsors, and especially Christopher Irwin, who has been our champion and guiding light on what a grid valuation framework can be and can accomplish. 


\section{Acronyms and Abbreviations}

AEO

ALR

ASHRAE

BAU

CAISO

CBA

CEII

DoD

DER

DG-IV

DOE

EIA

EIPC

FERC

GAAP

GMLC

IRP

ISO

LOLP

LSE

MCT

MISO

MWh

NEMS

NERC

PUC

PV

QCT

RI

RPS

RTO

SAG

SAIDI

TPL

TVA
Annual Energy Outlook

adequate level of reliability

American Society of Heating, Refrigerating and Air-Conditioning Engineers

business as usual

California Independent System Operator

cost-benefit analysis

Critical Energy Infrastructure Information

Department of Defense

distributed energy resources

distributed generation-integrated value

US Department of Energy

Energy Information Administration

Eastern Interconnection Planning Collaborative

Federal Energy Regulatory Commission

generally accepted accounting principles

Grid Modernization Laboratory Consortium

Integrated Resource Plan or integrated resource planning

International Organization for Standardization

loss of load probability

load serving entity

model characterization table

Midcontinent Independent System Operator

Megawatt-hour

National Energy Modeling System

North American Electric Reliability Corporation

public utility commission

photovoltaic

question characterization table

Rhode Island

renewable portfolio standards

regional transmission organization

Stakeholder Advisory Group

System Average Interruption Duration Index

transmission planning

Tennessee Valley Authority 


\section{Contents}

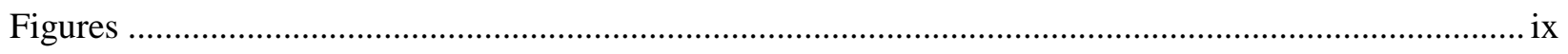

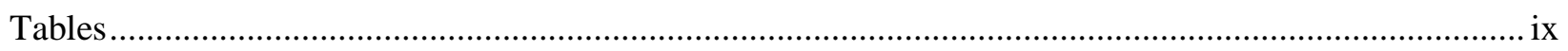

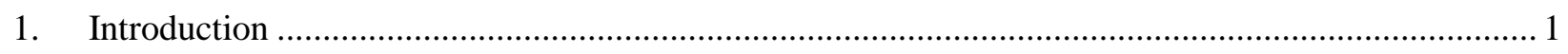

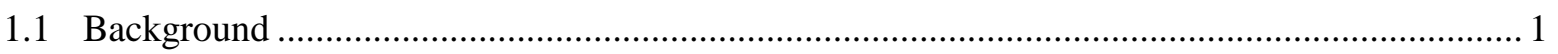

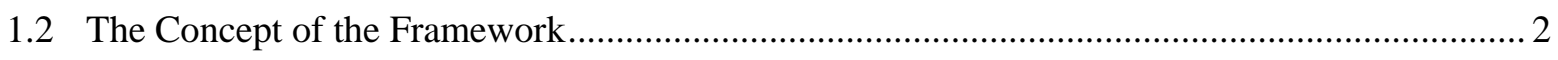

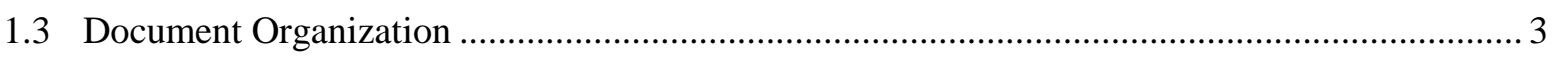

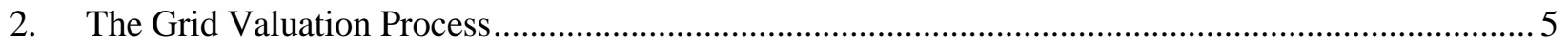

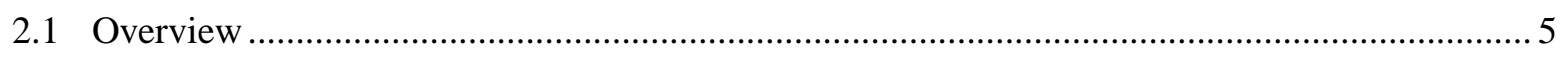

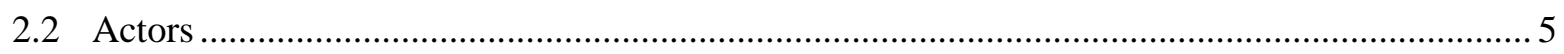

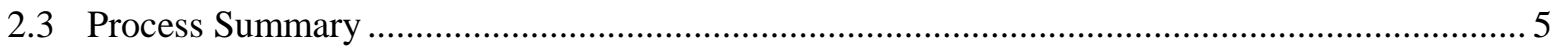

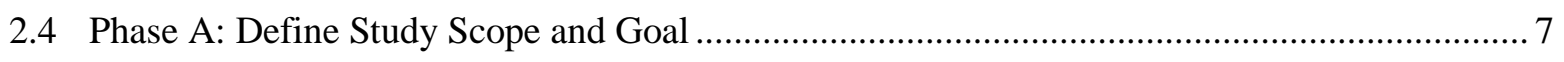

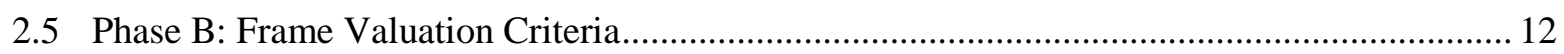

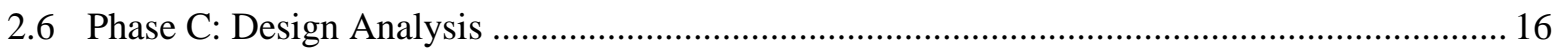

2.7 Phase D: Determine and Present Results ............................................................................... 19

3. Framework for Valuation of Grid Modernization Alternatives ................................................... 22

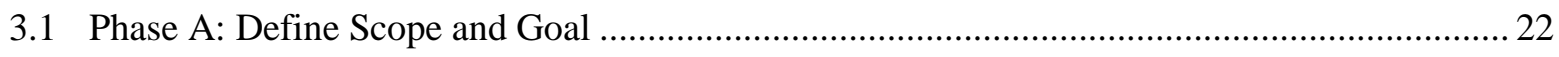

Step 1: Define Valuation Context and Purpose ................................................................... 22

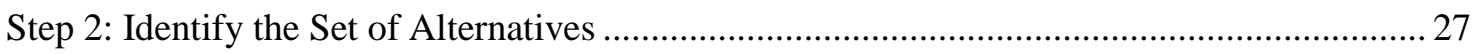

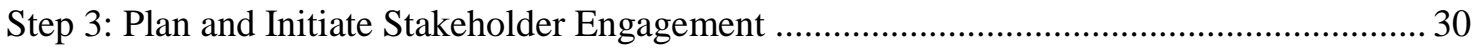

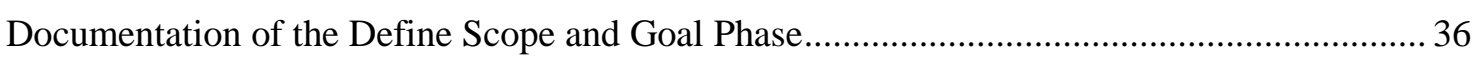

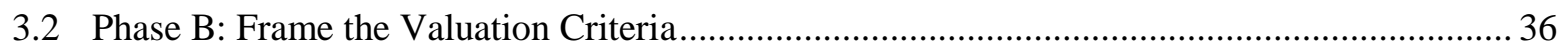

Step 4: Identify Relevant Impacts and Metrics .................................................................. 36

Step 5: Determine Multi-Criteria Integration Approach ....................................................... 40

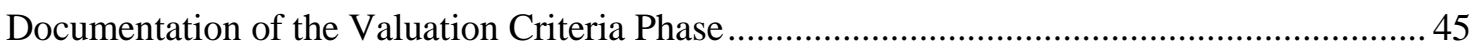

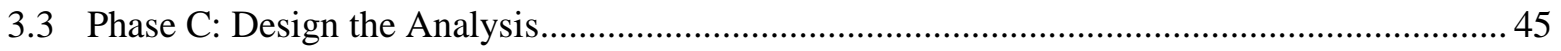

Step 6: Determine Approach to Address Uncertainty ......................................................... 45

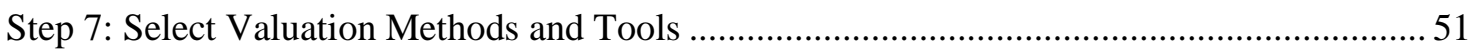

Step 8: Develop Assumptions and Input Data...................................................................... 56

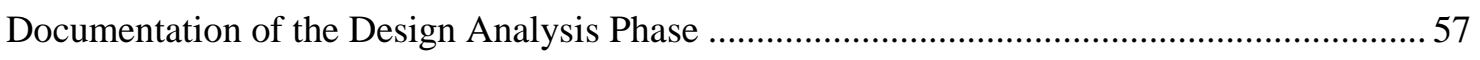

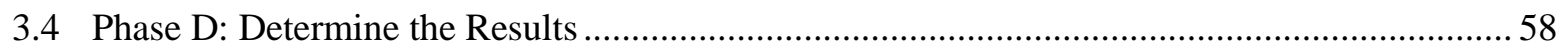

Step 9: Assess Impacts for Each Alternative ........................................................................ 58

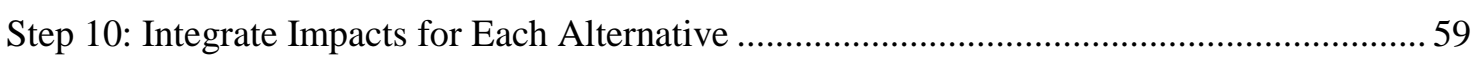

Step 11: Compare Values, Document Analysis, and Report Findings .....................................59

Documentation of the Determine and Present Results Phase.................................................. 61

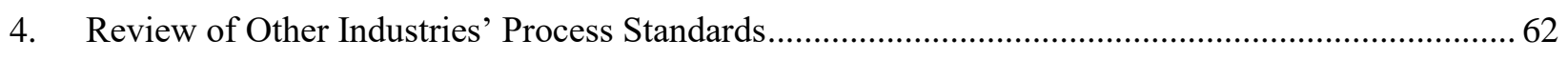

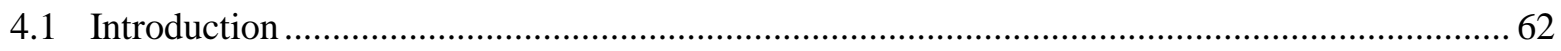




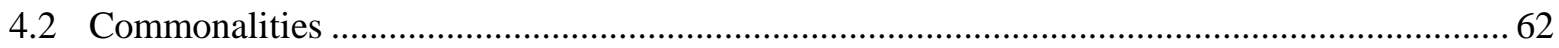

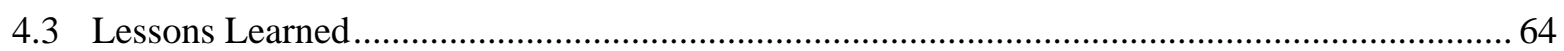

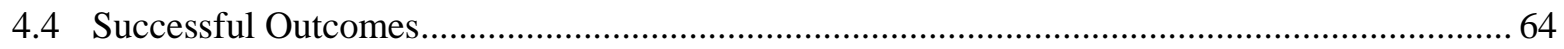

4.5 Considerations for Increasing Consistency of Grid Valuation Processes ............................... 65

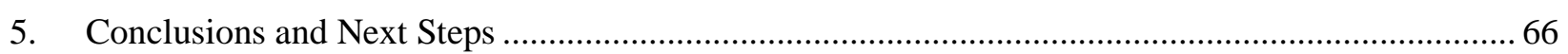

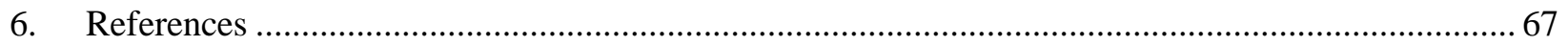

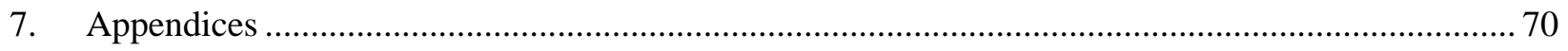




\section{Figures}

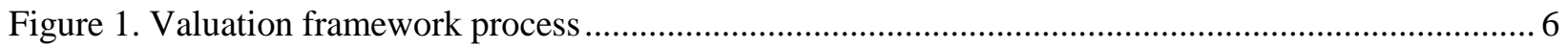

Figure 2. Example of stakeholder perspectives on components of TVA's DG-IV calculation methodology

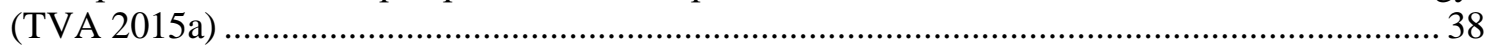

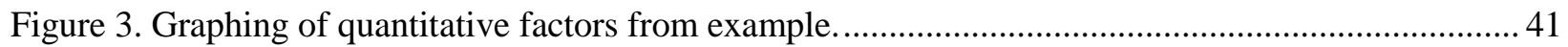

Figure 4. Cross comparison of two impact metrics from the EIPC transmission study. (EIPC 2011) ....... 42

Figure 5. Illustration showing case where using expected value of inputs does not lead to true expected value of outputs for nonlinear systems..................................................................................... 49

\section{Tables}

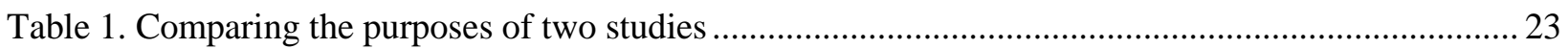

Table 2. Example schedule of milestones and deliverables for a recent valuation study......................... 24

Table 3. Example of impacts from different alternatives...................................................................... 40

Table 4. Heat map table of some of the metrics from the EIPC transmission study. (EIPC 2011) ............ 43

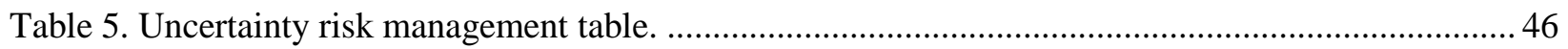

Table 6. Techniques to manage uncertainty in valuation (adapted from Wilkerson et al. 2014)............... 47

Table 7. Questions to be answered in final documentation ...................................................................... 60 



\section{Introduction}

\subsection{Background}

Across the electric power industry, a diverse stakeholder community applies a variety of methodologies to assess the value of courses of actions, ranging from investments in generation, transmission or distribution assets; to compensation mechanisms for distributed technologies; to a multitude of other policies related to projects in the electricity sector. The resulting value assessments often vary widely for the same or similar types of projects. As an example, two studies addressing the value of rooftop solar photovoltaic (PV) installations came to significantly different conclusions ${ }^{1}$; the studies chose different valuation metrics and analysis methods. This example is not unusual: often valuation studies are not comparable or repeatable because of differences in assumptions with respect to economic and engineering inputs; time, geographic, and power system scales; the closed-source inner workings of modeling tools and analysis methodologies employed for the valuation; stakeholder-specific choices of the key metrics or grid attributes that are the focus of the valuation; and/or choices of which grid investment, design or operation alternatives to compare. Investment decisions and policy options are reviewed and interpreted by multiple stakeholders, who have different motivations, interests, and obligations.

The values of a grid design option can no longer be expressed by just using historical cost-emissionsreliability metrics. Nor can major decisions be made through closed analysis without consideration of the different stakeholders affected by a decision. This valuation framework provides a structure for valuation that is deliberate, inclusive, and transparent, documenting the intermediate and final results to ensure clarity, accountability and repeatability.

This document, herein called Valuation Framework Guidelines, describes the guidelines for the framework for valuing grid generation, transmission, and distribution assets - including distributed generation and storage - and the services they provide, as well as policy options commonly evaluated in the context of the electric power grid. The framework is the primary product of the US Department of Energy's (DOE) Grid Modernization project titled "Grid Services and Technologies Valuation Framework Development," also known by its project number, GMLC 1.2.4. A team of national laboratory personnel have organized its development through a review of existing valuations and standards, experience with valuation exercises, and discussions with a Stakeholder Advisory Group (SAG) comprised of industry, regulators, and other organizations.

The field of accounting has a systematic set of generally accepted accounting principles (GAAP). Broad adoption of GAAP by professionals promotes consistency among analysts that facilitates apples-to-apples comparisons and ensures accurate, objective, verifiable and repeatable reporting of financial status. Similarly, the GMLC 1.2.4 project team envisions a long-term aspirational goal of establishing a set of generally accepted valuation principles for application to grid decisions that may in the future be adopted by stakeholders in the electricity sector.

The Valuation Framework Guidelines represents a first step toward that aspirational goal. It seeks to address inconsistencies, lack of transparency, and intrinsic biases often inherent in grid-related valuation studies. There are a great diversity of technologies, options, decision makers and other stakeholders involved in valuation studies; it is not realistic to expect that one standard method could be developed to evaluate grid options applicable to such a wide range of issues and technologies. Instead of a prescriptive

\footnotetext{
${ }^{1}$ E.g., Trabish, Herman K., "How two value-of-solar studies add up to no clear value of solar: Is rooftop solar really worth 90\% less in Montana than in Maryland?" Utilitydive.com, May 10, 2018, https://www.utilitydive.com/news/how-two-value-of-solar-studies-add-up-to-no-clear-value-of-solar/522892/
} 
methodology, the Valuation Framework Guidelines proposes a set of elements, processes, and internalconsistency checks that could improve the ability to value grid services, technologies, and policies comprehensively, objectively and transparently.

The framework embodies a systematic approach to the definition and documentation of the scale, scope, and assumptions that influence any grid-related valuation or modeling activity. It is intended to improve the ability of electricity-sector decision makers, analysts, and stakeholders to conduct, understand the basis of, and interpret the results of valuation studies, with high levels of transparency, repeatability, and extensibility. Such outcomes should also enable more effective comparisons of results among similarly constructed studies in a way that enables improved leveraging of prior work.

\subsection{The Concept of the Framework}

"Valuation" in this context is the process of determining the relative worth, utility, or importance (i.e., value) of options or alternatives to allow their comparison in ways that are clear, transparent, and repeatable. "Value" does not refer only to monetary savings and costs. Value may encompass multiple elements and associated physical measures such as reliability, resiliency, flexibility, sustainability, security, environmental quality, public health and safety, and economic impact. To support this document, Appendix A lays out an abstracted definition of valuation, describing key concepts and defining key terms that are used throughout this document.

Metrics are a means to express attributes and performance measures that constitute this broad definition of value. Metrics may be quantitative or qualitative. Identifying quantitative metrics for some of the elements is straightforward. However, in many cases, defining metrics (especially qualitative metrics) may be far less clear and may be controversial. Externalities (effects or consequences that are not explicitly included in the costs attributed to a grid option under evaluation) can also be very difficult to define and quantify but may be extremely important factors in grid-related decision making. Because it can be difficult and controversial to define many quantitative or qualitative metrics, it is important to be explicit about how all metrics are defined, are gauged, and contribute to the valuation.

The GMLC team has drawn on the use of software development frameworks to apply the concept of a "framework" for valuation in the electricity sector. Software development frameworks identify what formal components are necessary in developing software to ensure an efficient process so that the resulting software meets the functional requirements. Similarly, the Valuation Framework Guidelines defines essential elements or activities for valuing grid alternatives to support decision making so that the valuation results support the information upon which the decision makers actually will base their choice. The long-term vision for this project is to construct a unified framework that accomplishes the following three interrelated goals:

- Goal 1: Develop a Grid Services and Technology Taxonomy and an associated glossary that documents and classifies services and technologies, the different types of products that these services and technologies bring to the power system, and metrics relevant and applicable to each.

- Goal 2: Describe Valuation formally, as an explicit Process, documenting the linkages among the methods, metrics, and perspectives outlined in the taxonomy.

- Goal 3: Develop Broadly Applicable, Stakeholder-Vetted Guidelines for the design, implementation, and documentation of a formalized valuation process.

The Valuation Framework Guidelines begins to address these three goals, with a focus on Goal 2, to assemble a framework for valuation of electricity sector alternatives. The development of a taxonomy is addressed by a glossary of terms used when characterizing and quantifying grid services and technologies. This glossary of terms can be found in Appendix B. 


\section{Attributes of the Framework}

The framework presented herein is intended to promote an informed and transparent analysis for gridrelated decision making. Just as important, the valuation process aims to assist when there is a rethinking or revisiting of previous decisions, perhaps in the face of practical resource constraints or iterative feedback from stakeholders. The framework is meant to be flexible and adaptable to focus on the problem at hand without being burdensome.

There will be considerable variation in how the valuation process is implemented. Analysts may use any of a number of modeling approaches or analytical tools. Similarly, factors such as uncertainty, data availability, and data quality will influence the accuracy of valuation analyses. These same factors could impose restrictions on the level of modeling detail or analysis that analysts or the organizations sponsoring the valuation analyses deem it worthwhile to conduct.

\section{Who is the Audience?}

The primary audience for this framework is those who specify, oversee, and use valuation studies (i.e., decision makers), helping them to make sure those who perform/implement the analyses are aligned with the decision makers' needs. As a consequence, the analysis community will find it useful and important to understand the framework process. Section 3 of this guidance provides greater detail on the process steps and would be useful for this audience. The broader audience includes a varied community of stakeholders: utilities, power generators and developers, wholesale market operators, regulators, local governments, and investors. Additional stakeholders have an interest in and will be affected by valuation studies conducted in accordance with the framework: electricity end-users and facility owners/operators, public interest groups, chambers of commerce and business associations, organizations concerned with public health and safety, etc.

The concerned stakeholders include groups that reflect varied roles, responsibilities, constraints, and interests. For example, a utility or generation company considering an investment ultimately must decide for itself to commit to an expenditure. Likewise, a public utility commission (PUC) may in fact be a decision maker that is constrained in the type, nature, and timing of its engagement in a utility-directed valuation study because of regulatory procedural requirements. Other organizations may use the framework to better understand the value of grid-related technologies or services and inform the public or decision makers.

It is important to acknowledge that institutional dynamics and vested interests influence the entire process, especially approaches to stakeholder engagement. The form of stakeholder engagement depends upon multiple factors, including the type of valuation and who is conducting the valuation (e.g., a commission-convened valuation, a valuation that a utility is doing on its own behalf to support a decision or proposal, or other entities interested in understanding and informing others on grid-related issues). Some stakeholders may have an overriding issue or objective and may not be swayed by any analysis or valuation comparison. While such stakeholder priorities should be considered to some extent during the valuation process, doing so in a constructive manner can be challenging.

\subsection{Document Organization}

The organization of the remainder of this document is as follows:

- Section 2 provides an overview of the 4 phases and 11 steps of the framework.

- Section 3 describes in more detail each phase/step and the activities to be performed, with the decisions or analyses to be made, suggested solicitation of stakeholder inputs, information to be gathered, self-consistency checks to be performed, and documentation needed during the course 
of the valuation process. Appendices to Section 3 include step-specific outlines, checklists, detailed guidance, and so forth that may be useful for particular valuation studies. This Valuation Framework Guidelines references some external resources, such as a catalog of electric system metrics (see Anderson et al. 2017), intended to provide practical assistance to applying this valuation framework and choosing from among possible approaches for conducting a valuation study.

- Section 4 draws upon other disciplines' development of process-specific standards and guidelines to discuss the lessons learned to transition the framework guidelines toward a possible industry standard.

- Section 5 discusses conclusions and possible next steps for the GLMC 1.2.4 effort.

- Section 6 presents the references used in the paper.

- A series of appendices are attached that provide additional background on valuation theory, a glossary of terms, a number of tables and checklists that will be helpful for various steps in the process, and the workbook developed to assist users during a valuation process. 


\section{The Grid Valuation Process}

\subsection{Overview}

Our goal of formally delineating valuation as a process is particularly challenging for three reasons. First, the process needs to be applicable to an enormous array of potential electrical grid-related decisions. Second, the process needs to be amenable to a wide assortment of users and implementers - the organizations that initiate a valuation, their stakeholders, and analysts, all with divergent resources, knowledge, experience, and so on - in situations where resources available for the valuation study may range from highly constrained to seemingly unlimited. Third, the process itself is complex, with many elements that unfold and interact over time in a manner that is only loosely sequential, with activities that are often simultaneous, interactive, and/or iterative.

In a valuation assessment, outcomes of two or more alternatives are compared on the basis of differences in their characteristics or metrics. The difference between the metrics of two or more alternatives is considered to be an impact, and impacts are then transformed into values based on the perspectives of decision makers. These perspectives have two primary components: (1) whether a given impact is a positive or a negative relative change, or neither, and (2) what decision-making criteria guide the ultimate way a value is reported and interpreted. The impacts of each alternative are presented in a way that informs the decision for which the valuation effort was conducted. Appendix B provides a glossary of the terms used to describe the valuation process in these guidelines.

When evaluating two or more alternatives, decision makers are often presented with arguments and information about alternatives that were derived from disparate methods and, as a result, may defy comparison. Decision makers also often need to address conflicting priorities among multiple stakeholders. The framework provides a guide for marshaling stakeholders, metrics, and evidence through the decision-making process in a systematic and transparent manner. Walking through a systematic process of scoping and conducting a valuation will create a consistent vision of how a valuation study should be executed. This same valuation process can also be used to interpret and assess valuation studies over which a reviewer has no direct control. By working through the same steps and applying the same systematic perspective and standards, users will add to the credibility and comparability of any study.

\subsection{Actors}

The framework process outlined in this section involves three sets of actors. The decision maker is the instigator of the study and recipient of the results or recommendations from the study, who will make the ultimate decision. The decision maker may be a single person (e.g., senior executive at a utility, energy services company, or public interest group) or a body of people (e.g., board of directors, regulatory board, legislative body). The second set of actors is the analysis team (analysts, modelers, consultants) that will conduct most of the detailed analysis. The team may be comprised of internal staff from the decisionmakers' organization, hired consultants or subcontractors, representatives from other interested organizations, or combinations of these. Third, stakeholders are those who are affected by the ultimate decision and want to have input in the valuation study. Each actor will have different roles and levels of involvement for the various steps in the framework process.

\subsection{Process Summary}

The valuation process starts when a decision maker decides to do a valuation study using the framework and ends with results and recommendations based on the information developed. In this section, we 
outline the 4 phases with 11 steps in this framework, we provide more detail on each step in the following sections. ${ }^{2}$

To present this complex valuation process in a tractable way, we organize it into two broad tiers: phases and steps. Phases are roughly sequential stages of the valuation process that depict major aspects of the valuation process. Steps outline the activities that are necessary to accomplish that phase of the study in practice. We suggest four main phases, once a decision has been made to conduct a valuation study:

A. Define the scope and goal-including articulation of purpose, alternatives, and description of stakeholder engagement;

B. Frame the valuation criteria - through identification of key metrics and means to address valuation based upon multiple criteria;

C. Design the analysis_-including methodology selection, input data, and treatment of uncertainty; and

D. Determine and document results_-including assessment, integration, and presentation in understandable form.

The 11 steps that span the 4 phases of the valuation framework process are identified in Figure 1.

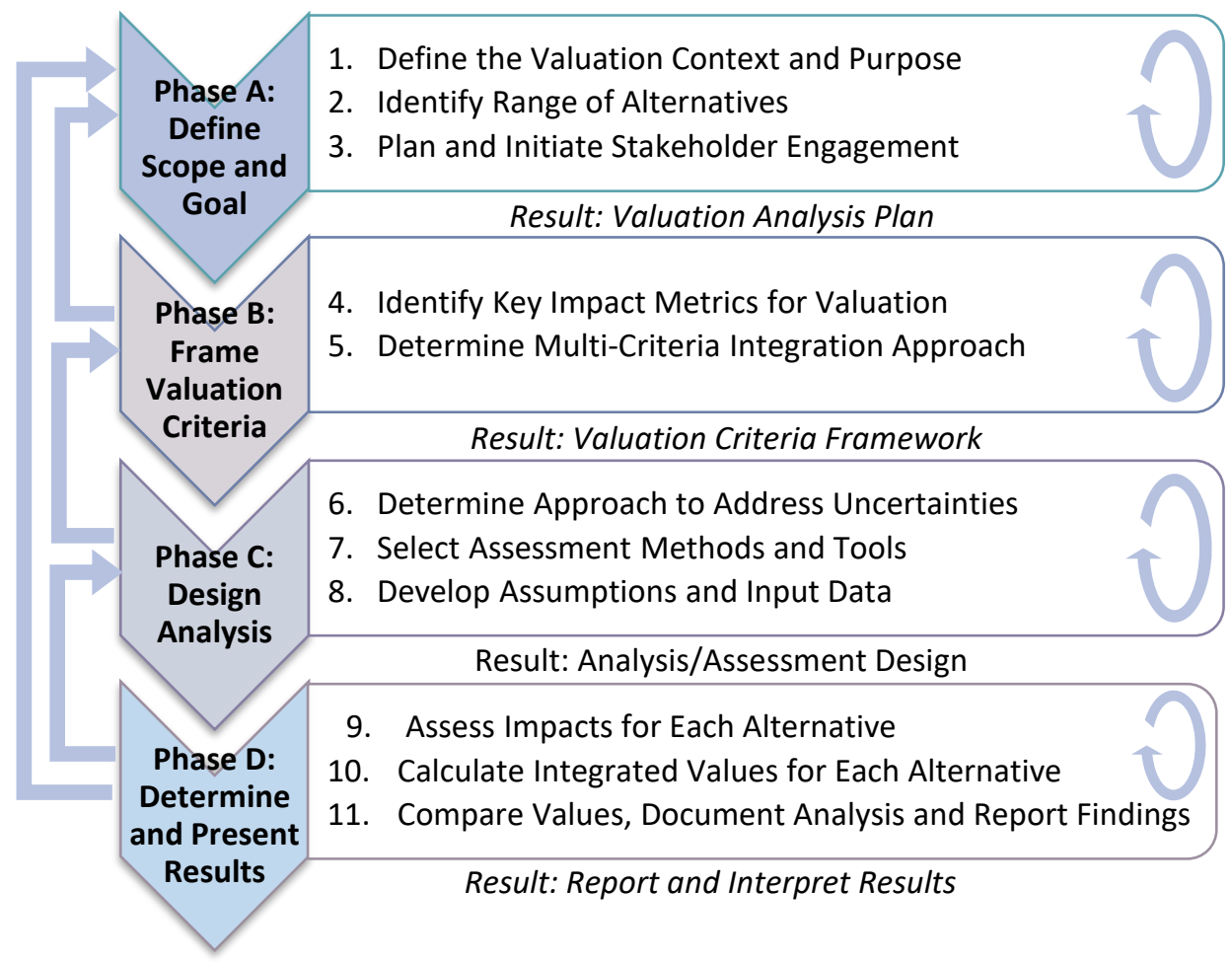

Figure 1. Valuation framework process

Conceptually, in phase A, the first three steps of the valuation framework can be considered the scoping phase of the study - carefully defining the purpose, context, relevant stakeholders, and alternatives to be considered.

\footnotetext{
${ }^{2}$ These steps and the following discussion are based loosely on Boardman et. al. (2006) and then refined and adopted for the purpose of the valuation framework by the GMLC team.
} 
In the next phase (B), defining the basis for the valuation, there are two steps to frame the information required to characterize alternatives (i.e., the impacts and metrics). In step 4, the users define the decision criteria for the study and then, in step 5, determine an approach to integrate or consolidate the metrics to portray the value of each alternative. (Note that "value" is often a complex concept and may not be reducible to a single number or a quantitative trade-off relationship among metrics.)

The third phase (C), designing the analysis, consists of three steps for constructing the study, including the tools, assumptions and input data (steps 7 and 8) to be used. This phase includes deliberately deciding how to consider uncertainty in the analysis (step 6) and consolidate predicted impacts from the different alternatives.

The final phase (D) is to calculate, analyze, interpret and present the results. The three steps in this phase are the calculation of key metrics; interpretation and presentation of the resulting values (steps 10 and 11); and reporting of the comparison among alternatives to guide decision making (step 9).

Many of the steps — such as characterizing stakeholder engagement - have multiple aspects, and their output will inform numerous other steps and several, possibly even all, of the four major phases. While the phases are generally sequential, the steps may not be. The analysis team may focus on activities in multiple steps simultaneously (or sub-groups of the analysis team may focus on different steps), and then the results or conclusions of some steps will further define the inputs of others. The results of one step may change the inputs or assumptions of a step listed previously (i.e., an iterative process). This iteration is shown by the arrows on the left and the circular arrows within the descriptors in Figure 1. The iteration is especially true for the first phase, where the study's objectives, scope, decision criteria, and rules of engagement are specified. To drive the valuation methodology process to a conclusion, metrics are a common currency for matching a question (Step 1) with appropriate tools and methods (Step 7) and providing the results presented (Step 11).

Assessments are complex and multi-variate. Determining the proper evaluation methodology (including the basis for evaluation as well as the selection of types of models) is as much an art as a science. The valuation process is far from being automated or even deterministic. The valuation framework can best be used by knowledgeable users. Thus, it is not designed to select models (or lists of eligible models) but rather to suggest the tools (including models) and analysis procedures to be employed.

The framework assumes that analysts are already knowledgeable regarding the grid system under study and details of the methods, tools, and models available. The user may well apply the valuation framework iteratively, choosing not only among models and tools but also among methodologies to calculate metrics and impacts, each of which may employ different sets of tools.

Documenting the decision process used to define the evaluation methodology and the reasons for choices made is key to transparency and promotes an alignment of objectives and methods that ensures valid and unbiased technical analyses. Appendix $\mathrm{J}$ provides a workbook for help in guiding the process through each of the eleven steps.

\subsection{Phase A: Define Study Scope and Goal}

The first phase involves defining the overall scope of a study and developing a plan for accomplishing the study (project work statement, resources needed, schedule, key milestones, and so forth.) After the initial decision that a study should be conducted, the framework begins with activities that include defining the study purpose and the external factors that are driving or influencing the rationale for the study; broadly defining the different options or alternatives to be studied; and identifying stakeholders, contacting them for the initial engagement, and planning for continued engagement. These three steps are tightly 
interrelated. Thus, in defining the scope, one also begins to identify the key stakeholders and possible alternatives to be considered.

Thus, the activities operate iteratively and somewhat in parallel, rather than sequentially. For example, an initial desire by a decision maker or decision-making body to conduct a study may kick off the entire process (Step 1), but relevant stakeholders may need to be consulted (Step 3) before the alternatives to be compared can be finalized (Step 2). ${ }^{3}$ In two illustrative studies cited below, decision makers consulted stakeholders at the beginning of the process to help define the scope and select alternatives:

- The Tennessee Valley Authority (TVA) first assembled a diverse stakeholder group (Step 3) to help scope the bounds of TVA's valuation and marginal rate-setting for distributed technologies (Step 1) (TVA 2015a). This set of stakeholders included representatives from rural and urban load-serving entities across the region, consumer groups, environmental groups, solar installers, and technical experts.

- In 2009, DOE sponsored in-depth transmission planning efforts for the three US electrical interconnections. The Eastern Interconnection Planning Collaborative (EIPC), a group of the eastern region's main transmission planning authorities, first created a Stakeholder Steering Committee [Step 3] to propose the alternatives to be considered [Step 2] (EIPC 2011).

\section{Step 1: Define the Valuation Context and Purpose}

\section{Objectives}

To define the purpose and scope of the valuation study and to develop an initial study plan.

\section{Approach}

Conceptually, the valuation process is initiated in response to a problem, condition, or issue that presents a choice regarding how best to proceed. Step 1 includes specifying the scope by defining the context and objective of the study in terms of the analysis question(s) to be answered. This step defines the purpose of the valuation; identifies the aspects of the power system to be examined; poses, in general terms, the issues that the valuation seeks to address; and sets the physical boundaries and the temporal range for the analysis. Documenting the issue or question and what one hopes the discussion/choice of alternative will accomplish - i.e., what constitutes a good outcome - helps ensure that stakeholders, decision makers, and analysts have consistent viewpoints about issues and alternatives.

While it may seem obvious, it is essential to document the circumstances, issues, and objectives that motivate initiating a valuation study. The discipline of succinctly setting down the issue to be addressed and what the decision maker hopes to accomplish (e.g., serve a growing demand for electricity at minimal cost while meeting reliability criteria) provides the basis for choosing which parts of the power system to examine, alternatives to be considered, stakeholders to contact, and performance metrics on which to base a decision. These choices will in turn inform the choice of methods and tools for dealing with uncertainty/unknowns, performing the analysis, and characterizing the results of the analysis.

Valuation addresses situations where there are multiple performance metrics (e.g., electricity price, land use, job creation, resilience) and the trade-offs among those metrics are not straightforward. Being explicit in what one views as a good outcome differentiates a valuation study (i.e., what is the value of choosing this alternative?) from a least-cost planning study (i.e., what is the lowest lifecycle cost?), although low cost will likely be one of the metrics used. The many alternatives available for expanding or

\footnotetext{
${ }^{3}$ An analogy is that when putting on a spare tire, you don't tighten one lug nut fully, then do the next, then the next, until all five are done. Rather, you tighten one nut lightly, then follow with light tightening of nuts 2, 3, 4 and 5 . Only after all five are in place and lightly tightened do you fully tighten each nut in turn. Similarly, exercising the steps in the valuation methodology is a round-robin process as much as a sequential one.
} 
upgrading the modern grid make it difficult to clearly and quantitatively define an objective that is likely to easily achieve consensus acceptance among stakeholders and decision makers.

One way to approach this step is to begin to document the scope of the study by formulating the problem and the system elements that it affects. This specification of study requirements (which will evolve and become more specific or detailed in subsequent steps) may include the following types of information:

- Primary purpose: i.e., aspect(s) of the power system one is trying to improve or optimize

- Area of interest (geographic).

- System(s), sectors (e.g., generation, transmission, distribution, end use), equipment or procedure(s) of interest (e.g., tariffs, generators: what is being modeled or evaluated — e.g., a feeder, a bulk power system, generators and loads).

- Time frame (start and end of time period of interest for implementing the alternative and evaluating its expected performance).

- Impacts: A preliminary list of relevant impacts and their metrics can be identified. However, key stakeholders (stakeholder engagement, Step 3) will further inform this list/selection and a more definitive list of metrics is developed in Step 4.

The analysis is constrained by the time and resources available to conduct the study. The study scope, number of metrics considered, and level of detail of the analysis must be consistent with the time and budget available. A detailed plan for the study must be developed, including schedule, tasks and milestones, expenditures, study participants and their roles and responsibilities, and list of stakeholders to be involved.

\section{Results/Documentation}

- A succinct statement of the purpose of the valuation, with context, including

$\circ \quad$ Whose perspective is primary (e.g., lead decision maker)

$\circ$ The purpose of the decision (e.g., policy development, investment)

- The time frame over which impacts are considered in the valuation

○ The geographic area for consideration

- A high-level specification of the overall process to be followed

- A preliminary list of stakeholders to be involved in the study

- An initial draft of the study plan, including time frame, budget, milestones, and evaluation team

- Identification of related studies that can inform selection of metrics and methods

- Sources of data to inform the analysis

\section{Interaction with other steps}

Steps 1,2, and 3 in the first phase are all closely interrelated. For subsequent phases, the characterization of the question and scope of analysis will inform the choice of metrics (Step 4), the relative weighting of metrics to influence the ultimate valuation-based decision (Step 5), and the nature and rigor of the models, tools, and analysis methods to be used (Step 7). The study plan developed during the first phase will govern all subsequent phases and their individual steps. 


\section{Step 2: Identify the Alternatives}

\section{Objectives}

To select which alternatives to analyze and compare.

\section{Approach}

In this step, the goal is to clearly identify the specific alternative courses of action that could be implemented. The scope of alternatives varies significantly based on the context of the decision. These can range from narrow specification of alternative policy implementations (e.g., require all utilities to meet a specific planning reserve margin) or specific investment projects (e.g., invest in new controls at an existing power plant), to portfolios of projects (such as utility integrated resource planning [IRP]), up to very broad analyses spanning all known technologies (in the case of national-scale techno-economic or policy modeling). Thus, alternatives can be policies, projects, portfolios of projects, or technologies.

Technological alternatives may be relatively easy to identify; however, their valuation may be more difficult, particularly if an alternative technology provides more or different value streams in addition to the primary one for which it is originally considered. For instance, a valuation comparison of distributed energy resources (DER) versus central generation plants will require fundamentally different analytical valuation approaches, as DER often provide added value to the customer. A central generation plant, in comparison, may primarily provide value to the bulk power system.
Defining Strategies and Alternatives The TVA Integrated Resource Plan (IRP) provides a good example of identifying a range of alternatives (Step 2 ), and assessing impacts of each alternative (Step 9). In the TVA IRP alternative courses of action are called "strategies" that represent business decisions that TVA can control. These strategies are then evaluated in multiple scenarios, which represent uncertain futures that TVA cannot control. In consultation with stakeholders, TVA identifies five distinct strategies that account for differences in desired attributes of resource portfolios. One strategy is the "Reference Plan" against which all other strategies are compared. Each strategy is then evaluated across several different metrics, including cost, risk, environmental stewardship, flexibility, and local economics. The results for each strategy and each scenario are then presented in a common scorecard for easy comparison across alternatives. TVA 2015b

In most cases, budget and/or time constraints limit the number of alternatives that can be considered. The number should be sufficiently large that alternative viewpoints and perspective are taken into consideration. It is important to ensure that all reasonable alternatives are identified and vetted. Transparency in the valuation process will support this: while the formulation of the basic question/issue and its scope (Step 1) usually suggests alternatives, a key aspect of this valuation framework is to examine the issue from other stakeholders' perspectives to see if nontraditional alternatives should be evaluated. One of the motivators for developing the valuation framework is that new technology developments, market models, and grid configurations offer innovative alternatives that may not be considered if one only looks at, for example, different types of central generation plants as options for meeting load growth. A diverse set of stakeholders can be the sources of several alternatives, often injecting novel ideas into the more conventional solution set. Indeed, using stakeholder engagement (Step 3 ) to propose alternatives has been successful in promoting acceptance of valuation studies (see the sidebar for the TVA IRP).

All valuations should have a baseline alternative against which to compare other alternatives. Typically, this baseline alternative is a business as usual scenario in which the state of the world follows current practice. The baseline alternative will include a description of the expected current path of broad societal and financial parameters (e.g., inflation, growth rates, legal policies) that will be consistent through all alternatives, unless specifically altered for exploration of various alternatives' robustness. 
Feasibility considerations are important - there is no need to evaluate an alternative if it cannot be implemented without violating some hard constraints (e.g., a renewable energy resource capacity may have to meet a utility's generation adequacy requirements).

Sometimes the attribute whose value is to be characterized is very complex, multi-faceted, and imprecise - such as power system's resilience. In such a case, characterizing the level of resilience could lead to a comparison of options for strengthening certain aspects of resilience.

\section{$\underline{\text { Results/Documentation }}$}

- Identification of the set of alternatives for consideration in the evaluation and boundaries.

- Specification of a business-as-usual case with which the value of other alternatives will be compared.

\section{Interaction with other steps}

This step also begins to specify the bases for comparison between alternatives. The specified bases for comparison (e.g., cost of electricity, emissions, reliability) will first be translated into the key high-level metrics (identified in Step 4). Weighting or prioritizing the metrics will be necessary to develop decision criteria to use in subsequent valuation steps (e.g., Steps 5 and 10). Stakeholder characterization and engagement (Step 3) will be a key determinant of this weighting. The nature of the alternatives to be considered will also inform the choice of methods and tools (Step 7), and the reasons for selecting the alternatives must be documented as part of Step 8 (assumptions and input data).

\section{Step 3: Plan and Initiate Stakeholder Engagement}

\section{Objectives}

Identify the stakeholders to be involved in the valuation study, define their roles, and develop a stakeholder engagement plan.

\section{Approach}

Stakeholder engagement is an integral part of the valuation process. Stakeholder engagement must be planned and initiated from the start, since it affects all the other steps. This step begins by identifying relevant stakeholders in addition to the organization initiating the valuation study (likely deemed the primary or principal stakeholder for the study). In this step, the entity conducting the study articulates the objectives, authorities and responsibilities of stakeholders in the valuation study as part of developing an engagement plan.

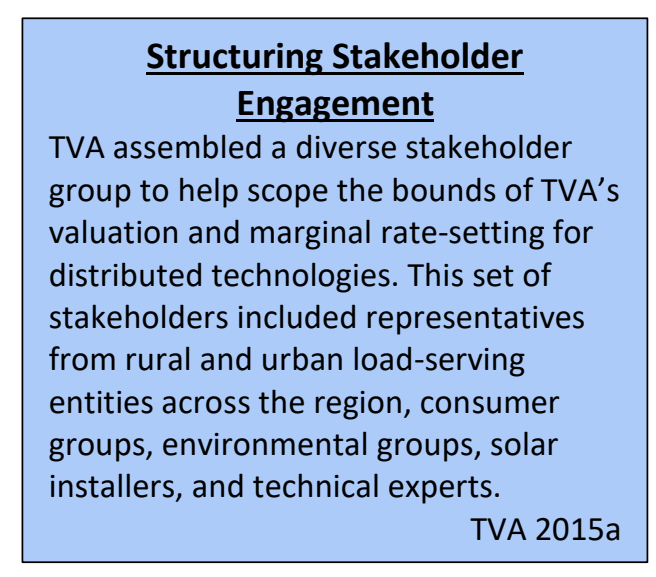

At the outset, the initiator of the study may postulate additional stakeholders' objectives and preferences based on the initiator's familiarity with each stakeholder's priorities and decision process, or on historical actions/decisions of a stakeholder. However, active follow-up and engagement with all relevant stakeholders is essential ${ }^{4}$ to help ensure that important analyses or metrics are not overlooked in the valuation and that any initial postulations about stakeholder perspectives are accurate. Meaningful engagement also may be necessary to ensure stakeholder buy-in to the option recommended by the

\footnotetext{
${ }^{4}$ Some level of cooperative engagement with stakeholders is important to obtain buy-in (and subsequent acceptance of results), but that may not always be possible, especially in existing forums likely to engage in valuation processes (PUCs, RTOs, FERC) that are often contentious and adversarial.
} 
valuation analysis. Stakeholders may be engaged through numerous methods, including direct polling or interviewing, advisory groups, holding workshops, and other, varied formal or informal interactions.

In some existing PUC, Federal Energy Regulatory Commission (FERC), and regional transmission organization (RTO) forums where many valuation study opportunities might lie, existing prescribed processes (regulatory or legislative) may inhibit formal involvement by regulatory bodies in the early stages of the analysis. For example, the PUC may be required to rule on a utility's final submission and thus may not be allowed to be part of the analysis team that crafted it. In such cases, absent changes to regulatory policy or procedures, the analysis team must anticipate and allow for the objectives or viewpoints of the regulator in implementing the valuation framework.

\section{Results/Documentation}

- List of stakeholders to be involved in the valuation effort.

- Stakeholder engagement plan, specifying areas of expertise and roles of each stakeholder. It should describe how stakeholder input shall be solicited or, alternatively, if some stakeholders are strictly adversarial or prohibited from direct cooperation (by legal or regulatory procedures), how the valuation study will consider these stakeholders' objectives.

- Plan for interacting with stakeholders.

$\underline{\text { Interaction with other steps }}$

Stakeholder engagement should be an integral part of Step 1 and Step 2 activities. But engaging with stakeholders, or anticipating stakeholder objectives or concerns, is part of every step. For this reason, anticipated stakeholder involvement is included in most valuation process steps in Section 3 of this document.

\subsection{Phase B: Frame Valuation Criteria}

The next two steps frame the required information (i.e., metrics) necessary to characterize and evaluate alternatives, decision criteria for the study, and the approach to integrate or consolidate the metrics to portray the valuation of each alternative (steps 4 and 5). (Value is often a complex concept and may not be reducible to a single number or a quantitative tradeoff-relationship between metrics.) Specifying the valuation criteria requires selecting the highest-priority metrics as well as quantifying or otherwise specifying trade-offs among metrics.

\section{Step 4: Identify Key Metrics for Valuation}

\section{Objectives}

To identify all the performance characteristics, impacts, and other metrics that will inform valuations, and prioritize those metrics according to their influence on the choice of alternative. Specify how higher-level or composite metrics (e.g., reliability, environmental quality, job creation) can be expressed.

\section{Approach}

Metrics used in valuation analyses are influenced by the nature of the question (e.g., if a PUC evaluates options by the resulting cost of electricity, then impacts on projected electricity prices are a metric) and by stakeholder input (e.g., if a utility requires that its generation plan include a loss of load probability [LOLP] of $<0.1$ day/year, then bulk power system reliability is a metric).

\section{Metric Selection Affects Solar Valuation}

Two recent studies on the value of solar show how considering a different set of impacts can influence the total value reported. A Montana study gave a total value of around $4 \mathrm{c} / \mathrm{kWh}$ but a Maryland study showed 30 $40 \mathrm{c} / \mathrm{kWh}$. According to one reviewer, "the most significant valuation differences come down to methodology and what is counted as costs and benefits."

Trabish 2018 
One resource that can be useful for identifying grid-related metrics is the Metrics Analysis reference document catalog developed by another GMLC project (Anderson et al. 2017). The document describes "existing metrics" and "metrics being developed." Since new metrics and new combinations of metrics are continually being developed, that report suggests that the valuation framework must allow stakeholders to propose new or customized metrics to reflect stakeholder-specific objectives for grid performance. Therefore, for the valuation methodology, metrics will be classified as follows:

- Base or fundamental metrics are the direct outputs of models and effectively "state variables" of the grid. Base metrics would include LOLP, system average interruption duration index, electricity cost (\$/megawatt-hour [MWh]), line loading (amperes), peak load (megawatts), sulfur dioxide emissions, energy/load forecast (gigawatt-hours/year), and others.

- Custom or composite metrics combine metrics and other quantities to provide information addressing complex evaluation criteria or decision processes. As an example, Anderson et al. (2017) cites an affordability metric of percentage of household income that is spent on electricity. This composite metric combines information on electricity expenditures by customers (e.g., forecasted consumption by end users multiplied by average retail electricity rates) with information on state-level household incomes for different types of residential customers. ${ }^{5}$

Strong feedback received from the SAG was that metrics should not be limited to grid metrics. The decision regarding which alternative to choose often depends upon metrics that are not inherently related to the power system or grid, such as the overall economic health of the region. An example would be whether less costly or more reliable electricity would attract additional industries to the area, and to what extent. Such "non-electric" metrics as job creation can be addressed similarly to power system performance metrics. Basic metrics such as electricity cost and reliability could be among the inputs to a regional economic model that forecasts compound or "mega" metrics such as job creation or unemployment rate. For a valuation study, the differences in basic metrics for each alternative (e.g., electricity cost per $\mathrm{kWh}$ ) could lead to different forecasts of economic indicators from a model of the region's economy.

The basic metrics and impacts needed for the valuation should be directly compared with the metrics and impacts that an analysis method or model provides as its output. Thus, it is necessary to match the analysis question's (Step 1) requirements for information with what candidate analysis models, tools, and methods (Step 7) can supply. Keeping this match in mind, Step 4 could use the following process to identify the basic metrics needed for the valuation:

A. Using stakeholder input/objectives (Step 3) and the scope of the question (Steps 1 and 2), identify all possible metrics and impacts to be reported/calculated as part of the valuation process. Use the metrics/impacts catalog (Anderson et al. 2017) as a guide for energy-related metrics. There may also be other non-power system metrics, such as economic indicators, that will influence the decision.

B. Determine (from all metrics in item A) which metrics or impacts are basic ones. Basic metrics or impacts are usually quantities that are direct outputs of a model.

C. If a metric identified in item A is not a basic quantity, then it is a custom or composite metric. Analysts should determine how that metric can be calculated from basic metrics and from other data. A list of basic metrics that compose the composite metrics will help to identify candidate

\footnotetext{
${ }^{5}$ The GMLC 1.1 Metrics Analysis Reference Document (Anderson et al. 2017) cites much ongoing research to develop, for example, forward-looking metrics that more effectively characterize resilience. The catalog of metrics developed by PNNL is valuable, but it is an evolving document, as the industry continues to develop new custom and composite metrics. Indeed, any new valuation exercise is likely to develop metrics customized to address the specific concerns and objectives of the involved stakeholders.
} 
methods and tools for the analysis. (Note that the sets of basic metrics that constitute different custom metrics will overlap.)

D. Prioritize the metrics and impacts that will be calculated. Each metric used in the valuation study requires resources - personnel, time, data gathering, models, and so on. It may not be feasible to evaluate all the metrics. Therefore, this step must also prioritize which metrics will be evaluated, for example:

- Key metrics essential for the valuation;

- Significant metrics that should be calculated if possible; and

- Metrics "of interest" that will not significantly influence the choice of alternative.

The study organizers can decide which metrics to calculate, based on their own objectives and resource constraints.

E. The methods/formulae for calculating the compound metrics from the basic metrics, data, and other composite metrics become part of the Multi-Criteria Integration Approach (Step 5), the Selection of Methods and Tools (Step 7), the Impact Assessment (Step 9), and the Value Calculation (Step 10).

\section{Results/Documentation}

- Comprehensive list of metrics that could be used to categorize the attributes to be valued in the study;

- Prioritization of the metrics to identify those that can be calculated within the scope, schedule and budget of the valuation study;

- Approach to calculate/estimate composite metrics from base metrics

Interaction with other steps

Metrics are the "mortar" that holds together the building blocks of each step in the valuation framework. The nature of the evaluation question and basis for making a decision (Step 1) and qualities that differentiate alternatives (Step 2) are used to identify metrics. Prioritizing the metrics directly informs Step 5, Multi-Criteria Integration. Determining how each metric shall be calculated, estimated, and/or simulated informs the identification of uncertainties (Step 6), choice of methods and tools to be used in the study (Step 7), and requirements for data and assumptions to be made in order to perform the analysis (Step 8).

\section{Step 5: Develop Multi-Criteria Integration Approach}

\section{Objectives}

The objective of this step is to develop a method to assign comparative values to alternatives characterized by disparate metrics. Not all metrics and decision criteria can be reduced to or expressed in a common unit (e.g., dollar cost).

\section{Approach}

This step develops and explicitly shows the trade-offs among impact metrics. In a complex valuation study, elements (metrics and impacts) usually interact. For example, a technology or design option offering enhanced grid reliability may also result in higher operating costs and may also have adverse environmental impacts. The valuation framework allows for framing trade-offs, such as those among electricity costs, reliability, and environmental impacts, so as to help identify the "best" option. Much of the delineation of trade-offs of metrics comes from the articulation of stakeholders' objectives (Step 3). In mathematical programming/

\section{Multi-Criteria Impact} Presentation

The EIPC study of the Eastern Interconnection involved more than 80 various scenarios and a large set of metrics for each. Several methods were used to consolidate results into meaningful presentations, including cluster diagrams using double variables, heat map tables that vary the color of individual cells based on their value, and transmission flow maps between the regions with width and direction of lines varying.

EIPC 2011 
optimization terms, quantifying such trade-offs would define the objective function upon which the valuation analysis is based.

The output of this step is a clear presentation of the valuation-related integration process. Trade-offs do not always need to be described quantitatively. For example, in comparing two alternatives with different cost and reliability impacts, one cannot objectively equate the values of the two (e.g., $\mathrm{X}$ change in LOLP is worth $\mathrm{Y}$ increase in electricity costs $\$ / \mathrm{MWh}$ ). It is often sufficient to present clearly the results of the alternatives in terms of impact metrics (e.g., cost, reliability, emissions, land use) as a basis for discussion and debate among stakeholders.

The multi-criteria integration may include constraints as well as trade-offs among impact metrics: any option studied may first have to satisfy certain requirements before trade-offs are considered. For example, power systems must first meet certain reliability requirements from the North American Electric Reliability Corporation (NERC) (e.g., critical infrastructure protection, adequate level of reliability [ALR] requirements), as well as regional, state, and local reliability requirements. The alternatives of a utility's resource plan options will result in various electricity costs and reliability and environmental impacts. Before the valuation framework is used to frame a debate about trade-offs among these three metrics (to identify the best option), all options to be studied will probably be required to first satisfy conditions such as:

- NERC's ALR requirements;

- $\quad \mathrm{N}-1$ or better contingency response (e.g., NERC TPL-001);

- LOLP of $<0.1$ day/year;

- Acceptable ranges for system voltages and equipment loading;

- Environmental Protection Agency emissions requirements.

Constraints and requirements may also be characterized as hard or soft. For example, a normal rating loading constraint can be soft (meaning it is undesirable but acceptable), but an emergency rating loading constraint is hard.

\section{Results/Documentation}

Methods to present and/or compare the values of alternatives as expressed by multiple metrics. If every attribute can be monetized, this task will be easier. However, because the methods and assumptions to monetize a metric may be controversial, the monetization methods must be documented. (e.g., what is the cost to the consumer of an electrical outage?)

Where multiple criteria cannot be reduced to a single common unit, the trade-offs can be shown (e.g., cost of electricity versus level of $\mathrm{SO}_{2}$ emissions). For some criteria, especially environmentally based or reliability-based criteria, there may be requirements that must be met (e.g., maximum allowed emissions). (See the sidebar on the EIPC's Eastern Interconnection study for examples of how to document multiple criteria to facilitate understanding.)

\section{Interaction with other steps}

The manner in which multiple criteria are viewed in the valuation process is influenced by the stakeholders (Step 3) and the metric prioritization (Step 4). In turn, the articulation of how multiple criteria are viewed for valuation analyses helps determine how integrated values are calculated for each alternative (Step 10) and how the results are presented and interpreted to support the decision-making process (Step 11). 


\subsection{Phase C: Design Analysis}

The three steps in this phase construct the study, including the methods, tools, assumptions, and input data to be used (Steps 7 and 8). This effort includes determining the methods to consider uncertainty in the analysis and consolidated impacts from the different alternatives (Step 6).

\section{Step 6: Determine Approach to Handle Uncertainty}

\section{Objectives}

To identify the unknowns and uncertainties inherent in forecasting, modeling, and valuation. To determine how to deal with and document these uncertainties (or estimates) in the analysis.

\section{Approach}

There are varied kinds of uncertainties that come into play during the course of valuation analyses, and that are associated with grid-related decision making that may be based partially or entirely on the results of valuation analyses. Illustrative categories of uncertainties include the following:

- Data-related uncertainties

- No data, or gaps in time-series data

- Incomplete or inaccurate data

- Forecasting future year conditions

- Price/cost-related: Future year forecasts of equipment/technology prices, fuel costs, labor costs, outage costs

- Capability-related: Uncertainties in technology development predictions (especially accounting for the results of current research and development) translate into uncertainty about equipment reliability/availability, performance characteristics (e.g., efficiencies, losses), operations and maintenance needs, other costs and prices

- Load or growth forecasts

- Event-related uncertainties: uncertainties regarding an event's occurring can include

- A forced outage of a generator, power line, transformer or other grid component

- Demand for power (i.e., load forecast, including time and spatial components)

- Costs-e.g., price of natural gas

- Occurrence or probability (e.g., frequency, magnitude) of an extreme event, such as a terrorist attack, solar flare/geo-magnetic disturbance, hurricane, flood, or earthquake

- Weather-temperature, rainfall, wind, solar incidence

- Power system-related uncertainties: uncertainty about the state of the power system (e.g., after an event, what components are out of service)

- Analysis method-related uncertainties: simulation and forecast analysis methods, tools and models can only estimate outcomes (impacts and metrics)

These kinds of uncertainties can be addressed through multiple methods (used singly or in combination):

- Use of expected value or most likely value

- Use of a range of values (e.g., high, medium, low forecasts)

- Stochastic techniques (probabilistic modeling), such as

- Monte Carlo analysis

- Probability density functions (e.g., Booth-Baleriaux method for production costing)

$\circ$ Probabilistic risk assessment

- Sensitivity studies 
- Contingency planning studies (e.g., loss of the first, second, third, etc. -largest generators or lines - contingency modeling is used to meet NERC ALR requirements; force 5 hurricane; highaltitude electromagnetic pulse event of $X$ magnitude)

- Definition of scenarios incorporating many assumptions and input values in a consistent manner (such as tabletop exercises).

Uncertainties can be treated as risks for the valuation evaluation processes. Risk identification and risk management/mitigation is a well-researched field. The Department of Defense (DoD) Risk Management Guide for DoD Acquisition, Sixth Edition (DoD 2006), provides a disciplined environment for proactive decision making to identify and mitigate risks.

The DoD approach results in a clear enumeration of identified uncertainties and a uniform format to describe how each uncertainty was assessed and addressed. This attention to uncertainty is an important differentiator from how many valuation studies are conducted. Often, the decision of how to deal with an uncertainty is made implicitly and never documented. Thus, a model may be chosen (e.g., for dealing with probabilistic uncertainty) or a mid-range forecast may be used (for dealing with predictive uncertainty about the future) as the customary way a consultant/analyst approaches that particular modeling exercise. However, the choice - and the reasons for it - may not be appropriate or accurate, and such a modeling flaw may not be detected because the choice of model/method was not documented.

This step usually requires that knowledgeable domain analysts familiar with the techniques for dealing with uncertainties, and their strengths and weaknesses, be engaged for this portion of the analysis. The value of this approach is that it clearly and transparently identifies uncertainties in the valuation process and explains how and why they will be considered.

\section{Results/Documentation}

Comprehensive documentation of the uncertainties inherent in the valuation methodology and the ways they are addressed.

\section{Interaction with other steps}

If specific modeling methods are chosen to deal with uncertainty, these methods can be compared with characteristics of potential tools and models to identify which models are compatible with the selected risk/uncertainty management approach (Step 7). The available budget and schedule (i.e., the study's resource constraints) as set forth in the analysis plan (Step 1/Phase A) will help determine the extent to which various uncertainties can be addressed.

\section{Step 7: Select Assessment Methods and Tools}

\section{Objectives}

To select appropriate analysis methods, tools, and models consistent with the information needs previously identified and feasible within the budget and schedule set for the study.

\section{Approach}

This step includes the selection of methods, models, and tools to calculate the impact metrics upon which valuation-related findings will be based. Models will be described or characterized using (but not limited to) the following information:

- Type of model or tool (e.g., load flow [static/dynamic], transient stability, reliability, contingency analysis, load profile, price elasticity, load forecast, revenue requirements, generation expansion, production cost, unit commitment)

- Subsystem/sector (e.g., generation, transmission, substation, distribution, microgrid/feeder, customer, load[s], combinations) 
- Geographic area or territory: World, North America, United States, interconnection, area served by a regional transmission or independent system operator, state-level area, utility, local area, substation, feeder(s), microgrid, campus, customer premises

- Time frame and resolution (micro-seconds to years), considering whether the focal grid-related valuation is for planning, for operations, or for developing operating procedures (planning for operations)

- Is the model for calculation (e.g., load flow, reliability) or optimization (e.g., production cost)

- Constraints in model calculation (e.g., equipment loading limits, reliability/LOLP required)

- Variables used for optimization (e.g., minimize fuel and operating costs)

- Model outputs (to be compared with basic metrics identified in Step 4): examples are loading, economics (capital and operating and outage), reliability (various indices and metrics), rates/tariffs, environmental effects

- Required inputs (e.g., are the data available, accessible, affordable?)

- Methods to handle uncertainty (e.g., stochastic techniques)

- Model ownership and licensing requirements.

Recognizing that valuation is a process, it is important to exercise consistency checks as part of that process to ensure that the analysis methods and tools do in fact accurately calculate the metrics and impacts upon which the decision (regarding which alternative offers the best value) will be based. The result of this step is a comprehensive and quantitative listing of the information requirements needed to perform the valuation analysis. The scope of the study (e.g., distribution system vs. bulk power system; utility service area vs. a city vs. a substation and its feeders) and the information required to choose among alternatives (metrics) inform what analysis methods and tools may be suitable for the valuation.

\section{Results/Documentation}

Documentation of a detailed calculation approach and data flows to provide the necessary metrics for a decision. The models and methods will have been chosen to ensure that the tools' purposes, capabilities, and outputs match the scope and requirements of the valuation question. (See the sidebar on MISO's multi-value transmission project for an example of good practice.)

\section{Interaction with other steps}

Models suggested for the valuation process will be those with the capability to calculate the basic metrics that have been designated as key. The characteristics of candidate models will be compared with the study requirements (as defined by Steps 1, $2,4,5,6)$. The methods to derive composite metrics from basic metrics (Step 4) and to integrate multiple criteria into the evaluation and decision processes (Step 5) provide the equations,

Documenting Selection of
Assessment Methods
Midcontinent Independent System
Operator (MISO) evaluated the
impacts of the Multi-Value
Transmission Project Portfolio, a
series of large transmission
investments whose costs are spread
across the North and Central regions
of MISO's footprint. This study
provides a clear example of how to
document the choice of assessment
methods and tools (Step 7) for
complex, multi-faceted studies.
data flows, and other methods to derive the required information from the basic metrics (i.e., model outputs).

\section{Step 8: Develop Assumptions and Input Data}

\section{Objectives}

To identify and document all sources of data and simplifications or assumptions made during the analysis. 


\section{Approach}

This step identifies the sources of data and documents any assumptions, simplifications or bounds on the models or tools used. Transparency about these selections is often crucial to the acceptance of the valuation; stakeholders will want to be able to examine the basis behind the assumptions and input data and potentially proffer alternate values. (However, care must be taken to protect certain Critical Energy Infrastructure Information (CEII) or confidential data sources.) The choice of assumptions about the state of the region and the power system and its customers will have substantial impacts on the quantitative results of the modeled alternatives. Even choosing initial values can be difficult. Power system models are complex, often requiring voluminous and detailed input data. The input data must be consistent across the different models employed in the valuation process. Care must also be taken that the outputs of a model have the proper units, resolution, and aggregation when they are used as inputs to another model.

\section{Results/Documentation}

A comprehensive list of data sources and assumptions made.

\section{Interaction with other steps}

The assumptions and key input data are influenced by the model and tool selection (Step 7) the metrics (Step 4), the means of comparing or presenting values based on multiple criteria (Step 5), and the handling of uncertainties (Step 6). The analyst team will decide which data source(s) to use. A literature search to find similar valuation studies (Step 1) may help identify data sources. In the course of executing this task, data may be unavailable or unreliable. In that case, an alternative approach may be needed, and the project team may have to go back to Step 7 to select a different model or analysis method; the new model must be checked against the study scope and information requirements to ensure it meets the information needs of the study.

\subsection{Phase D: Determine and Present Results}

The three steps of the last phase are the calculation of key metrics; interpretation and presentation of the resulting values; and reporting of the comparison among various alternatives to guide decision making.

\section{Step 9: Assess Impacts for Each Alternative}

\section{Objectives}

To exercise the analysis methodology and calculate the impacts/metrics of each alternative.

\section{Approach}

Conceptually, this step is straightforward: simply perform the analyses using the methods, tools, models and input data previously selected. With the valuation study modeling framework and assumptions assembled, the chain of models and analyses are executed to produce quantified impacts of different metrics across the range of alternatives defined in Step 2, including the baseline or business-as-usual case. The models, simulations, and cases must also reflect the methods chosen to deal with uncertainties (Step 6), possibly including additional scenario analyses, sensitivity analyses, and/or Monte Carlo simulations. The impacts should be quantified over the full time-horizon for which the actions and alternatives are expected to produce differences with the baseline case.

\section{Results/Documentation}

The expected impacts and characterization of each alternative, as calculated using the tools, data and methods of the previous steps.

\section{Interaction with other steps}

The metrics and impacts will be used to calculate the value of each alternative (Step 10) using the multicriteria integration approach developed in Step 5. 


\section{Step 10: Calculate Integrated Values for Each Alternative}

\section{Objectives}

To present the metrics and impacts for each alternative that are used to assess its value.

\section{Approach}

This step calculates integrated values for each alternative based on quantification of alternatives using the multi-criteria approach defined in Step 5. If tools such as surveys and interviews are used, their more qualitative results may need to be integrated with the quantitative modeling outputs before values for each alternative can be determined.

Depending on the method of integration and communication, this task can be as simple as totaling financial estimates, or as complicated as soliciting priorities, ranks, and weights from a variety of stakeholders. Variations on the integration criteria may be applied to better understand the perspective of various stakeholders or simply the robustness of the integrated value. Results after applying uncertainty factors can also better inform the fully integrated results for each alternative.

\section{Results/Documentation}

The combined elements of expected impacts of each alternative necessary to express its relative value.

\section{Interaction with other steps}

The valuations of each alternative will be compared in the final step.

\section{Step 11: Compare Values, Document Analysis, and Report Findings}

\section{Objectives}

To present the relative valuations of each alternative in a format that facilitates objective comparison by decision makers and other stakeholders. To prepare a final report and complete comprehensive documentation of the valuation study.

\section{$\underline{\text { Approach }}$}

This step documents the findings, including the opportunity to publish a matrix of metrics, if appropriate, rather than try to combine all metrics into a single valuation number/index/metric. Step 1 (Stakeholder Engagement) and Step 5 (Multi-Criteria Integration Approach) inform the format and content of the presentation of valuation findings. Steps 8 (Assumptions and Input Data), 9 (Calculate Impacts), and 10 (Calculate Integrated Values) determine the numeric values.

\section{Results/Documentation}

The final report and documentation of all methods, data and assumptions upon which the evaluation was based. (See the sidebar on DOE's WindVision study and its communication of results.)

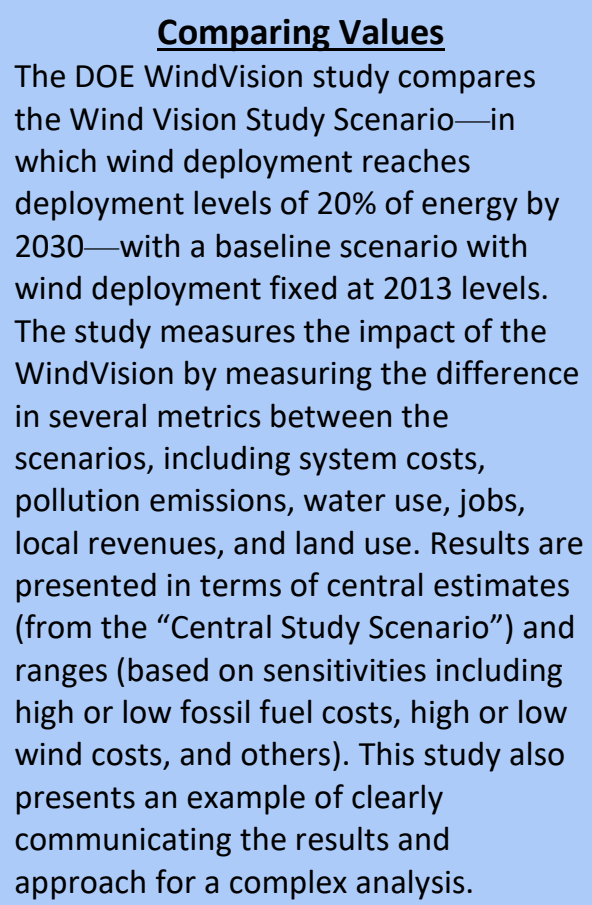

DOE 2015 


\section{Interaction with other steps}

Each step has documentation requirements that, taken together, should in theory fulfill the needs of this final step. In fact, it is expected that the documentation produced previously in the process will be reviewed for completeness and consistency. 


\section{Framework for Valuation of Grid Modernization Alternatives}

\subsection{Phase A: Define Scope and Goal}

The first of the four major phases in the process involves defining the overall scope of the study. After the initial decision that a study should be conducted, the framework begins with activities that include defining the study purpose and the external factors that are driving or influencing the rationale for the study; creating a study plan; broadly defining the different options or alternatives to be studied; and identifying stakeholders, contacting them for the initial engagement and planning for continued engagement. The activities operate iteratively and somewhat in parallel, rather than sequentially. For example, an initial desire by a decision maker or decision-making body to conduct a study may kick off the entire process (Step 1), but relevant stakeholders may need to be consulted (Step 3) before the alternatives to be compared can be selected (Step 2).

\section{Step 1: Define Valuation Context and Purpose}

The purpose of the valuation guidance is to provide a framework for comparing the relative attractiveness of alternative courses of action in a transparent and repeatable manner. Critical to the success of later steps of the valuation process is the clear articulation of the context and purpose for the valuation effort, expanding on the analysis question that initiated the study. This articulation is useful both as a guiding document as the valuation effort is being executed - to ensure that the various actors within the study have a consistent vision of its purpose - and as a record for interpreting the valuation effort once its results are summarized and released. The decision maker (person or body) leads this step with the assistance of the analysis team once the team has been identified and selected. Engagement with major stakeholders is also important in establishing the context and purpose of the valuation effort.

The structure of this documentation may be guided by chronicling/recording the answers to a series of questions about the valuation effort. We give a sample of questions below, but questions should be adapted to most clearly articulate the relevant points of each particular valuation effort. Note that not all questions require an explicit answer before a valuation study is initiated-it can also be helpful to articulate questions that are unknown at the outset and must be determined over the course of the study.

- From whose perspectives are the valuation assessments being performed? For example, ratepayers? A utility? Society? Is one perspective primary? Since the same alternatives can have differential impacts on various stakeholders, the perspective of the valuation can impact which alternative is most attractive.

- What specific decision is the valuation is intended to support, if any? If it is intended to support a specific decision, what is the decision and who is (are) the decision maker(s)? The purpose of valuation can run from high-level investigation of policy options to real-world investment decisions potentially affecting grid infrastructure. The stated purpose of the valuation process can carry implications for the minimum levels of breadth and depth that are necessary to carry out the valuation analyses credibly. In some cases, a valuation study might be used to compare alternative futures, without supporting particular decisions that would be required to achieve those futures. Clarifying this upfront enables transparent comparison between valuation studies that might have differences in purpose. Table 1 compares a study designed to support a specific decision with one that evaluates alternative futures without supporting a particular decision. 
Table 1. Comparing the purposes of two studies

\begin{tabular}{|l|l|l|}
\hline Purpose & \multicolumn{1}{c|}{ DOE Wind Vision } & \multicolumn{1}{c|}{ TVA 2015 IRP (TVA 2015b) } \\
& $\begin{array}{l}\text { Analyze feasibility, costs, and benefits of } \\
\text { increased wind power deployment to } \\
\text { inform policy decisions at the federal, } \\
\text { state, tribal, and local levels }\end{array}$ & $\begin{array}{l}\text { Guide TVA in making decisions about the } \\
\text { energy resources used to meet future demand } \\
\text { for electricity through 2033 }\end{array}$ \\
$\begin{array}{l}\text { Alternatives } \\
\text { Compared }\end{array}$ & $\begin{array}{l}\text { A Study Scenario with 10\% by 2020, 20\% } \\
\text { by 2030, and 35\% by 2050 wind energy as } \\
\text { a share of national end-use electricity } \\
\text { demand is compared against a Baseline } \\
\text { Scenario where wind energy is frozen at }\end{array}$ & $\begin{array}{l}\text { Five distinct planning strategies that were } \\
\text { assessed over the 20-year IRP planning } \\
\text { horizon, including a Reference Plan. } \\
\text { Strategies represent future business } \\
\text { decisions over which TVA has full control. }\end{array}$ \\
$\begin{array}{l}\text { 2013 levels } \\
\text { Specific }\end{array}$ & None & $\begin{array}{l}\text { Recommendation of target resource mix for } \\
\text { Decisions }\end{array}$
\end{tabular}

- Is the valuation effort restricted to a particular technology, investment, or policy compared against a baseline? A common first-order bifurcation of valuation studies is whether they are focused on a particular pre-determined alternative, or if they are intended to be a general assessment of multiple alternatives, not all of which may be identified at the outset.

- What are the key factors and environmental forces? It is typically useful to identify the external drivers that are motivating the valuation effort. These drivers can include the current state of energy supply and demand, bottlenecks, upcoming changes to laws or the environment, technology changes, and others. Describing the broader external conditions can help facilitate the identification of alternatives and key metrics throughout the evaluation process.

- What resources are needed to conduct the valuation? Depending on the purpose of the valuation, an analysis team and other resources will be needed to support the valuation process. This team could expand or contract as the purpose evolves through iteration. For example, if the purpose expands in scope, then computing, legal, accounting, environmental, or other specialties may be needed. It may be necessary to hire outside specialists to assist in conducting the valuation analyses. This team will take the lead on most of the remaining steps with guidance from (and within the financial, temporal, and other constraints imposed by) the decision maker(s) funding the valuation process.

- How should the valuation process be documented and the results disseminated? Will the results be made public? (Some analyses and results may be confidential for a variety of reasons.) In a formal evaluation process, each step should be documented to capture and communicate the rationale used. This documentation should be done from the start to avoid attempting to recollect earlier steps later in the evaluation. Predefined deliverables for each step also may improve the quality of the valuation.

Note that this valuation guidance document underscores the importance of documenting the context and motivation for the valuation process. At a minimum, this documentation compels the party sponsoring the valuation study to articulate explicitly its goals and scope. Additionally, a clear articulation of the context and motivation for the valuation can help ensure that the final results are actually useful and in support of the grid modernization decisions that may have prompted the valuation. Because of the importance of stakeholder engagement throughout the valuation process, this step is also a good time to document the stakeholder engagement plan. 


\section{Detailed Guidance for Planning the Overall Process, Timeframe, and Milestones}

The overall process of a valuation study can take significant time and resources in cases when a high degree of precision is needed, a wide range of alternatives are considered, or the scale of the analysis is large (e.g., large national or regional studies). In contrast, simpler or narrower analysis can be used in cases where speed is essential or resources are otherwise limited. Planning the valuation study should therefore start with establishing the time when the decision or findings are needed and the resources (funding and personnel) that can be dedicated to the project. As an example, one study of the value of solar done at the state level with a high level of detail had a budget for consultants of $\$ 500 \mathrm{~K}$ and a timeline of over 1 year. With fewer resources available, a similar question could be addressed by using a range of estimates from related studies or consultation with experts. A study with a budget of $\$ 50 \mathrm{~K}$, for example, could be used to identify aspects of the valuation that are most likely to impact the relative comparison of alternatives, providing a clear scope for more detailed analysis. On the other hand, having more resources and time can allow for activities to increase the confidence in results, such as pilot studies to validate aspects of the analysis.

Depending on the scale of the valuation study, it may be helpful to have multiple opportunities for the analysis team to interact with and check in with key stakeholders and decision makers. In planning the study, it is therefore important to establish milestones and deliverables at multiple points through the process. An example of such a set of milestones and deliverables from a recent valuation study is reproduced in Table 2.

Table 2. Example schedule of milestones and deliverables for a recent valuation study.

\begin{tabular}{|c|c|}
\hline Date & Analysis team identified by the project manager \\
\hline +15 days & In-person scoping meeting with commission personnel \\
\hline +40 days & Study outline presented to commissioners and/or project manager \\
\hline+50 days & Study outline approved by commission and/or project manager \\
\hline+150 days & $\begin{array}{l}\text { In-person meeting to present draft report to commission and commission } \\
\text { personnel }\end{array}$ \\
\hline +155 days & Draft report, as edited, provided to stakeholders \\
\hline +175 days & Stakeholder comments due \\
\hline+185 days & Draft of final report presented to commissioners and project manager \\
\hline+190 days & $\begin{array}{l}\text { Commissioners and project manager provide written comments, if any, to } \\
\text { incorporate into the final report }\end{array}$ \\
\hline +195 days & $\begin{array}{l}\text { Final report published after written approval from project manager and presents } \\
\text { final report to commission in public meeting }\end{array}$ \\
\hline
\end{tabular}

Developing a schedule of milestones and deliverables for a particular valuation study can be aided by answering the following questions:

- What resources (funding, personnel) are needed to answer the question? At a high level, consider the importance of having rigorous, detailed, comprehensive analysis compared with relatively coarse approximations. The resources used to study a question should shift according to the magnitude/impact of the investment or policy being considered. The level of detail and the resources needed to determine an optimal investment portfolio for a utility are likely far larger than the resources needed to consider a non-wires alternative at a single location on the distribution system.

- When does the valuation need to be completed? The timeline of a study depends on the urgency of the decisions informed by the analysis. Decisions about subsidies required to prevent 
pending retirements of power plants might be more urgent and require tighter timelines than decisions about broad-reaching grid modernization programs that might roll out over many years. The timeline may also be based on resource availability (e.g., using existing staff versus contracting a third-party) and the availability of data.

- What major milestones are required? Establishing milestones helps to ensure that progress is being made in the analysis and provides an opportunity for stakeholders and decisions makers to provide feedback and correction during the process. Major milestones might include meetings with key stakeholders and decision makers to determine the scope, meetings and documentation establishing the valuation criteria, proposed analysis, draft reports of the analysis results, presentations to stakeholders, and documentation of final results.

- How should the valuation process be documented? Clear documentation of the valuation process increases transparency and reproducibility. Meetings are helpful for eliciting feedback and communicating with key stakeholders, but written documents are longer lasting and provide a clear useful guide to help others understand the valuation process. Documents can include interim reports, final documentation, spreadsheets or databases of underlying data and assumptions, or other forms of documents.

\section{Learning from Related Studies}

During this first step of the valuation process it should also be useful to examine related studies and document the findings from those studies. Previous studies of related questions, even if conducted for other geographies or focused on comparing other alternatives, can provide useful information for scoping valuation studies, identifying key impacts and valuation criteria, designing analytical approaches, and conveying results. The degree to which resources are dedicated to identifying and cataloging related studies depends on the overall scale of the valuation process, but it is important to keep in mind that extracting insight from related studies often requires less effort than reproducing similar results. Related studies are not the only source of information for developing a valuation study. While learning from past studies can be insightful, it should be recognized that past studies may not have been accurate; and other forms of information and research - including engaging with stakeholders and experts - should also be considered. A goal of this project is to foster valuation studies that are consistent and extensible, adding to the body of relevant literature available to future analysts.

Documenting the findings of related studies can focus on summarizing individual studies or on comparing how particular steps of the valuation process are addressed across studies. The findings should be conveyed beyond the analyst team to include informing decision makers, stakeholders, and other subject matter experts.

\section{Stakeholder Considerations}

There are two main aspects of stakeholder engagement during Step 1 that initially may seem contradictory. First, stakeholder engagement is crucial to the Step 1 objective of articulating the valuation context and purpose. Second, valuation analysts, perhaps in conjunction with the grid modernization decision maker(s), should develop a stakeholder engagement plan for the full course of the valuation process.

One goal of directly engaging some of the major stakeholders at the beginning of the study process is to solicit their perspectives in shaping the purpose, alternatives, and bounds covered in the valuation study (see sidebar below). Initial transparency in the scoping of the valuation study can prevent costly iterations through the valuation process and subsequent delays in making an informed decision. 
EXAMPLE: The Tennessee Valley Authority (TVA) assembled a diverse stakeholder group to help scope the bounds of TVA's valuation and marginal rate-setting for distributed technologies. This set of stakeholders included representatives from rural and urban load-serving entities across the region, consumer groups, environmental groups, solar installers, and technical experts.

TVA Distributed Generation-Integrated Value (DG-IV) methodology (TVA 2015a)

\section{Whom to Engage-Key Potential Challenges}

A key challenge is to engage stakeholders effectively before developing a plan for engaging stakeholders. One approach is to start by identifying potential stakeholders for the grid modernization project at issue according to the generic stakeholder engagement process previously discussed. This set of stakeholders then can help shape the valuation purpose sufficiently for analysts to be able to develop a stakeholder engagement plan for the remainder of the valuation process. The initial set of grid modernization project stakeholders can help set the boundaries of the valuation study and for valuation-related stakeholder engagement. Should a project-related committee or similar group already exist, as in the example cited in the sidebar below, valuation analysts can tap it to achieve Step 2 stakeholder objectives.

GRID MODERNIZATION DECISION-MAKING EXAMPLE: In late 2009, DOE sponsored in-depth transmission planning efforts for the three electrical interconnections that cover the continental United States. As set forth in the DOE statement announcing the project, this planning was to "develop an open, transparent, and collaborative process that will involve participants from industry, federal, state and local government agencies, universities, and non-governmental organizations." To organize its project, the Eastern Interconnection Planning Collaborative (a group of the region's main transmission planning authorities) began by creating a Stakeholder Steering Committee with voting representatives from the region's states (through their own Eastern Interconnection States Planning Council), industry representatives, and consumer and environmental organizations. Only after this committee was convened were the range of alternatives and sensitivities proposed and developed.

(EIPC 2011)

There is a caveat to that approach, which constitutes another key challenge. The structure of an existing group (i.e., who is or is not a member of it), and its history and dynamics can shape the advice provided. It can shape the specification of alternatives, the delineation of the purpose of the valuation, and which stakeholders and impacts are deemed "relevant." Advice from this initial set of stakeholders may be excellent, but it also may be constrained or embody implicit biases that could prove counterproductive in achieving the valuation goals. One approach to dealing with this challenge is to ensure that early stakeholder engagement (Step 3) is highly iterative during Steps 1 and 2.

A related challenge is to select a set of stakeholders for this early stage of engagement when there is no preexisting stakeholder committee or similar group. The composition of the set of stakeholders consulted matters because it affects which viewpoints are - and are not-tapped.

\section{Additional Resources}

Appendix C includes some templates, checklists, guidance, and tools that may be useful for accomplishing Step 1: a checklist for scoping the purpose; an example of a resource consideration matrix; a list of questions for organizing a review of related studies; and an outline for the deliverable summarizing the purpose, process, timeframe, and milestones.

\section{Step 1 Deliverable:}

- A succinct statement of the purpose of the valuation, with context including the following: 
- Whose perspective is most important,

- The purpose of the decision (e.g., policy development, investment)

$\circ$ The timeframe over which impacts are considered in the valuation

$\circ$ The geographic area for consideration

- A listing of the overall process to be followed

- A description of the overall stakeholder engagement plan

- An initial timeframe and budget for the process, the initial evaluation team

- Learning from related studies and how that information should be used in subsequent steps

- Major milestones for the rest of the valuation

\section{Step 2: Identify the Set of Alternatives}

After defining the context for the valuation effort, the next step is to clearly identify the specific alternative courses of action that could be implemented. The scope of alternatives varies significantly based on the context of the decision. These can range from narrow specification of alternative policy implementations (e.g., require all utilities to meet a specific planning reserve margin) or specific investment projects (e.g., invest in new controls at an existing power plant), to portfolios of projects (such as utility IRP), up to very broad analyses spanning all known technologies (in the case of national-scale techno-economic or policy modeling). For the remainder of this document, we will refer to various courses of action generically as "alternatives," noting that in practice alternatives can be policies (inclusive of rate-setting, market rules, and the variety of local, state, and federal-level regulations and incentives), projects, portfolios of projects, or technologies.

Technological alternatives may be relatively easy to identify; however, their valuation may be more difficult, particularly if an alternative technology provides more or different value streams in addition to the primary one for which it is originally considered. For instance, consider DER as an alternative to a central generation plant technology. A side-by-side valuation comparison of these two generation resources will require fundamentally different analytical valuation approaches because DER provide value to the customer and potentially provide value and/or cost to the distribution system, as well as-if coordinated by aggregators - bulk power values. A central generation plant, in comparison, may claim value only for the bulk power system.

Policy alternatives, generally, include variations of central policy thrusts to explore the sensitivity of a set of goals or constraints to the overarching policy objective. Many valuation studies have been performed to explore the costs and benefits of certain levels of RPS for a particular jurisdiction.

In most cases, the number of alternatives considered for a valuation is small, and they should be chosen wisely. The number should be sufficiently large that alternative viewpoints and perspective are taken into consideration. Usually, a diverse set of stakeholders can be the sources of several alternatives, often injecting novel ideas into the more conventional solution set-for instance, using battery technology as an alternative for traditional transmission upgrades (Puget Sound Energy 2017).

As mentioned in the discussion of taxonomy, all valuations should have a baseline alternative against which to compare other alternatives. Typically, this baseline alternative is a business-as-usual scenario in which the state of the world continues to follow current practice. Note, however, that this does not mean that a business-as-usual scenario is exactly the same as the current environment-just that any anticipated decisions follow current practice. An important consideration in the valuation process is understanding that the power system must be adaptive over long time scales (simulating and approximating this adaptation is a key concern in the selection of modeling methods, tools, and assumptions in Steps 7 and 8) and that alternatives proposed in the valuation process should reflect this reality and must be feasible. The US Energy Information Administration (EIA) Annual Energy Outlook (AEO) provides a sound example of the definition of a baseline case (called the reference case). It assumes that a technology advances 
under predictable and understandable rates, and it considers current and future national and state legislative requirements. For instance, it implements future RPS requirements as they are set by each state (EIA 2016).

Feasibility considerations are important - there is no need to evaluate an alternative if it cannot be implemented without violating some hard constraints. The feasibility requirement can be explicitly imposed on an alternative; for instance, a renewable energy resource capacity must meet or contribute to a utility's generation adequacy requirements. However, the feasibility of an alternative cannot always known in advance, such as in the case of a utility's IRP process. The following paragraphs describe a series of steps an analyst should take to derive the set of (feasible) alternatives to be considered in the valuation process.

\section{Defining Constraints and Feasibility}

Readily identifiable, well-defined, and feasible alternatives to compare against business-as-usual conditions can be difficult and fraught in practice. There are multiple sources of potential infeasibility, and it is common to over-constrain alternatives. The following principles and considerations are intended to help guide that process:

- A distinction must be made between hard constraints (legally or jurisdictionally binding rules and regulations) and soft constraints such as cost, risk, time, or difficulty thresholds. It is easy to confuse the two. Hard constraints necessarily bound the definition of feasible alternatives. Soft constraints may point to alternatives and insights that may otherwise be overlooked or assumed away. Furthermore, soft constraints should be represented and explored to the extent possible in the modeling framework (e.g., as additional costs or as sensitivities). Being explicit about assumptions with respect to what issues constitute hard and soft constraints is a fundamental issue of transparency and can facilitate productive discussion with stakeholders. To the extent soft constraints can be exceeded or traded off between other value categories, they may be more appropriately included within the value equation rather than constraints per se.

- Hard constraints may eliminate certain alternatives (e.g., certain projects or technologies) from consideration entirely - but that is not necessarily the case. Instead, they may simply set the boundary conditions for the broader suite of alternatives.

- Alternatives compared in the valuation process are not necessarily guaranteed to be a priori feasible. Often, feasibility considerations are explicitly engineered into the methods, tools, and assumptions used in modeling the impacts of valuation. For example, capacity expansion models may explicitly address the resource adequacy aspect of balancing demand with supply (plus reserve margin). But they do not guarantee that the generated power of the new capacity can be delivered through the transmission system to the load centers. Only additional power flow analysis will reveal the feasibility of delivery.

Setting reasonable physical boundaries of the analysis is important. It places a constraint on the assets to be considered in the analysis. Usually, larger geographic footprints consider greater numbers of assets, and with that, a greater degree of freedom to find optimal solutions. Frequently, policy options are defined by states having jurisdiction over the electric generation assets. Thus, a natural boundary would be the state borders. However, from a power flow perspective, the transmission system is governed by laws of physics (Kirchhoff's law); thus, larger contiguous networks such as interconnections may be considered as the scope for the analysis. The final decision about the reasonable physical constraints should be based on the data availability, computational capabilities, and characteristics of the technology/or policy to be valued. 
Systematically determining the spectrum of alternatives is likely to motivate a rethinking of how the decision under consideration is defined. Therefore, the decision maker and analysis team should be prepared to revisit and modify the purpose posed in Step 2. Although an initial list of the alternatives to consider is necessary to proceed with subsequent steps, the list may change. For example, a stakeholder not yet involved in the process may prompt the decision maker or team to include or exclude a particular alternative. All three steps are involved in designing the scope of the valuation, as indicated in Figure 1, and will interact with each other.

\section{Additional Resources}

Appendix D includes some checklists that may be useful for accomplishing Step 2: a checklist of potential hard constraints and a checklist to help set boundaries.

\section{Stakeholder Considerations}

Stakeholder engagement in Step 2 aims to help identify the set of alternatives to be considered in the valuation analysis. Again, a diverse set of stakeholders usually yields a more diverse set of alternatives.

Particularly for valuation efforts that are open to considering new alternatives as they arise, the first two activities may not be considered complete until stakeholders have weighed in on the valuation purpose, key environmental factors, or alternatives. However, in many cases, the alternatives to be studied will be determined before the valuation effort and any associated stakeholder engagement are initiated formally.

\section{Whom to Engage-Key Potential Challenges}

Steps 1 and 2 may occur simultaneously or nearly simultaneously. In these situations, whoever participated during Step 1 will participate in identifying alternatives for the valuation analysis. This situation increases the importance of, and the potential complications associated with, the choice of which stakeholders to engage during Step 1. For example, deliberations about which alternatives to include or exclude and the specification of those alternatives requires specialized expertise. Some categories of stakeholders may be excluded from this early engagement because their influence or expertise is insufficient to allow them to provide input that is meaningful in selecting the set of alternatives or (in Step 1) articulating purpose of the valuation. A decision to exclude some stakeholders may be sensible and pragmatic when viewed from a technical valuation-analysis perspective, but it could prove problematic if the exclusion of (some of) these stakeholders becomes a matter of contention.

When Steps 1 and 2 occur far enough apart in time, it is possible to plan in a deliberate manner to select stakeholders with desired technical or experiential expertise for alternative selection and specification. The set of stakeholders engaged in Step 2 may be the same as those in Step 1, a subset of those stakeholders, or affiliated with different portions of the same organizations represented earlier. Step 2 may require stakeholders and subject matter experts (who may not be defined as stakeholders) who did not participate in Step 1. As in Step 1, the selection of which stakeholders to include and exclude can shape the decisions made during Step 2 and can have ripple effects throughout the valuation process and possibly the grid modernization decision-making process.

Note that analysts, and possibly the grid modernization project sponsor or decision maker, will have to decide what constitutes "desired expertise," though these decisions can be made in consultation with others (e.g., stakeholders). Regardless, this decision is impactful for the valuation effort and associated grid modernization decision making.

A potentially major challenge is to establish and convey the role that stakeholders will play in deciding which alternatives to pursue during the valuation process. It is possible that the stakeholders who are engaged may influence but not determine these choices because of such factors as resource limitations 
and grid modernization decision maker obligations and constraints. Misunderstandings or inconsistent expectations about who will decide upon alternatives and what role involved stakeholders will have in that decision making can lead to friction and distrust during and after Step 2. Related to this challenge is deciding how to resolve disagreements or disputes among stakeholders regarding which alternatives to pursue.

\section{Step 2 Deliverable}

- Identification of the set of alternatives for consideration in the evaluation and boundaries

- Identification of assumptions and input data for evaluation of each alternative

- Input to Step 4-Identification and prioritization of evaluation metrics

- Input to Step 7-Identification of the assessment tools and methods

- Input to Step 8-Documentation of assumptions and input data

\section{Step 3: Plan and Initiate Stakeholder Engagement}

\section{Stakeholder Engagement as an Integral Part of the Valuation Process}

Stakeholders and stakeholder engagement are integrated into the valuation process, but details and emphases vary in different steps. In practice, there is a range of standard practices in place across the nation, from fairly routine use of non-evidentiary processes to engage people, especially appropriate for innovation-based issues, to states where a non-evidentiary process is unusual. This section of the valuation framework provides a high-level overview of how stakeholders and stakeholder engagement function throughout the valuation process. It starts by introducing and defining key phrases and concepts and then summarizes a generic stakeholder engagement process. Subsequent sections describe stepspecific details, emphases, and considerations. Appendix D provides associated tools and checklists.

Who are the stakeholders? By some definitions, it can be hard to exclude people or organizations from potential lists of stakeholders - e.g., a stakeholder can be defined as anyone who is interested or invested in something and/or is impacted by and cares about it. In a broad sense, grid modernization project or policy proposers are stakeholders; as are people or entities who make decisions about projects or policies; as are governmental and nongovernmental organizations, businesses, and people who live or work in potentially affected areas.

There are multiple junctures during the valuation process where either the grid modernization project sponsor or the valuation analysts need to determine which stakeholder(s) to involve, in what ways, and to achieve which ends. Judgments about which stakeholders to involve and how are context-dependent and therefore can differ at various stages of the valuation process. In some cases, decisions about whom to include and exclude in stakeholder interactions can communicate intentional or unintentional messages about issues like transparency and inclusion. These decisions can help set the tone for stakeholder engagement, whether in positive ways or in ways that stir distrust or exacerbate controversy.

Grid modernization-related decision making and stakeholder interactions take place in an historical context. The past influences the present (whether directly related to the issue at hand or not). Smooth operations, respectful and productive interactions among stakeholders, and trusted players combine to create an entirely different context for later decision making and stakeholder interactions from a history of operational problems and antagonism or distrust among key players. In the latter case, the new decisionmaking and stakeholder involvement processes may need to be designed partially to overcome past problems and build trust. Similarly, the valuation process and grid-related decision making occur over time, so the nature of stakeholder engagement during earlier valuation steps can affect the issues and nature of interactions during later steps. 
Past and current interactions also can be affected by particular individuals' personalities and proclivities. Stakeholder engagement for new grid-related decisions may include these same individuals, and therefore build upon past patterns of interaction, whether those patterns were positive or fraught. The introduction of new players (e.g., individuals in leadership or other key roles) can introduce uncertainties and disrupt past patterns of interaction. Regardless, any new stakeholder engagement for valuation processes or gridrelated decision making should be attentive and responsive to key individuals and patterns of interactions among stakeholders in the past that can affect current interactions.

Utilities and other entities may have their own, well-established stakeholder engagement approaches and practices. These approaches may be informed by federal, state, regional, and local regulatory agencies and designed to align with varied corporate or organizational functions and goals. Thus, some of these approaches may emphasize public-oriented outreach and feedback. Such approaches may serve more to meet public relations objectives than to help guide valuation analyses and subsequent decision making.

Stakeholder roles are not uniform; for example, PUCs are unlike many other interested parties in that they have regulatory authority and are often in fact the decision makers that choose among grid modernization alternatives. In some existing PUC, FERC, and RTO forums where many valuation opportunities might lie, existing proscribed processes (regulatory or legislative) may inhibit involvement by regulatory bodies in the early stages of the analysis; i.e., the PUC may be required to rule on a utility's final submission and not be allowed to be part of the analysis team that crafted it. In this case, absent changes to regulatory policy or procedures, the analysis team must anticipate and allow for the objectives or viewpoints of the regulator in implementing the valuation framework. Fortunately, PUCs' objectives and priorities are usually well known from past rulings.

This guidance document tends to portray stakeholder engagement as a set of formalized processes to tap the perspectives of such grid-related stakeholders as grid infrastructure asset owners, utility commissions, public interest representatives, or technology original equipment manufacturers or third-party providers. However, established stakeholder engagement approaches and practices may not currently be implemented for valuation-related analyses. The generic guidance in this document can still complement these other approaches. And it may be particularly useful for entities or interested parties without such well-developed approaches, or in cases where those approaches have not been implemented for valuation analyses.

\section{Determine and Articulate Goal(s) of Stakeholder Engagement}

In planning for stakeholder engagement, it is essential before beginning the engagement to articulate one or more specific goals for that engagement. Stakeholder engagement goals should be specific and attainable, at a minimum, even though the goals may be a subset of higher-order project goals or aspirations. That is, "modernizing the grid" is a higher-order goal that is too broad for these stakeholder engagement processes. Some may wish to specify SMART goals—specific, measurable, achievable, results-focused, and time-bound.

Valuation informs decision making but is not the sole determinant. As such, stakeholder engagement within the valuation process can be substantially different from that used for the final decision making. There naturally will be overlap between the two purposes for stakeholder engagement and in the individuals and groups participating as stakeholders. Therefore, it may be especially important to articulate the stakeholder engagement context and goal(s) at several junctures throughout the valuation process. In other words, there may be an overarching goal for stakeholder engagement but particular subgoals associated with different steps in either the valuation or grid modernization decision-making process. 
Note that stakeholder engagement can take many different forms, associated with different contexts, goals, players, and sometimes what can be labeled philosophical approaches. The latter category can be manifested in many ways - ranging from thinking of stakeholders as groups to be informed vs. groups to be consulted vs. active decision makers. Some forms of stakeholder involvement are consistent with a participatory governance philosophy. In these cases, stakeholders (sometimes including members of the public) actively help to define the goals.

\section{Identify What Constitutes "Success"-Gauging Interim and Outcome Success}

It is essential to know when, or the extent to which, specified engagement goals were achieved. Thus, it is important when specifying a goal also to define what "success" means and how to gauge that success or know it when one sees it. It also is important to identify how to gauge whether the engagement step is on track to achieve its goals, to indicate where refinements or corrections in approach are needed.

EXAMPLE: A goal of DOCUMENTING KEY ISSUES OF CONCERN to stakeholders is substantially different from a goal of MAKING A DECISION THAT IS ACCEPTABLE to most/all stakeholders involved in the decision-making process. Success for the former could take the form of a comprehensive list of issues of concern that "all" interested stakeholders have had the opportunity to vet, refine, and approve. Success for the latter could include a plan/decision that aligns with (or addresses) the formal obligations and restrictions among "all" stakeholders who need to approve, buy into, or implement that plan/decision.

\section{Identify Important Stakeholders for Achieving Specified Goal(s)}

The ideal is to identify all stakeholders who are relevant to particular goals. In some case, the number of potentially relevant stakeholders is so vast that is may be virtually impossible to include all —especially when members of the public are included among the relevant stakeholders. The following are some issues to consider in identifying "relevant" stakeholders.

- Draw (initial) boundaries around who is and is not affected by the potential decision and to what extent. The earlier specification of stakeholder engagement goals can provide a framework for making determinations, though discussions of potential stakeholders can, in turn, help refine the goal articulation, too. Recognize that initial sets of stakeholders may expand or contract over time and throughout the process. Think about the relative importance of stakeholders:

$\circ$ Within and outside of the sponsoring or decision-making organization

- At local, regional, or national levels

- With direct and indirect roles or interests (e.g., parties that do/don't have decision-making authority, associations that may represent a broad swath of interests but not any particular entity's positions or likely actions)

EXAMPLE: The electric grid is a highly connected network that, in turn, is connected to many other environmental (e.g., rivers and the atmosphere), manmade (e.g., natural gas pipelines), and socioeconomic (e.g., industry and employment) networks and systems. Actions or events at one location can induce impacts across multiple geographically dispersed interconnected systems at time scales that can be immediate or on the order of decades. For a quantitative evaluation of alternatives, valuation analysts should articulate which stakeholders should be considered in conducting analyses to identify indirect impacts of alternative grid modernization-related decisions. 
EXAMPLE: While a state utility commission may consider impacts to ratepayers and utilities to be of highest importance, it is primarily only concerned with impacts to its own ratepayers and utilities, although a policy or rate decision may create impacts that propagate into neighboring states or interconnected competitive markets.

- Think broadly about which stakeholders should be engaged and to what extent. This is often not automatically or immediately obvious from lists of interested parties, interest groups, interveners, etc. It can be important to try to address this from multiple perspectives. Examples of questions to ask include (also see the illustrative sidebar below):

- Which stakeholder groups are essential to achieve the specified goal?

- Early and later phases

- Kind of role - decision-making, implementation, maintenance, operation, oversight, other form of affected party

- Which stakeholder groups can impede or block the achievement of those goals?

- Which stakeholders associated with different systems can affect the achievement of specified goals?

O Other interconnected systems such as cyber- or communications systems, water management systems, transportation systems, and so on

- Other regulations or guidelines such as building codes, union rules, and so on

- Consider who (or what perspectives) stakeholders do/and do not represent, and who is included vs. excluded from participation either deliberately or inadvertently. Judgments about these issues are context-specific, dependent on the scope, scale, and particulars of issues at hand. But, in general, organizations are not monolithic. It can matter whether the individual(s) who are the involved utility or industry stakeholders come from the contracting or legal department vs. an engineering or operations department. Similarly, the public and categories like non-governmental organizations or the business community are far from singular.

- Map key stakeholder roles, responsibilities, requirements, and perhaps spans of authority as they relate to the specified engagement goal(s) and overall project goals. There may be statutory or similar restrictions in what different stakeholders are or are not allowed to do that are relevant throughout the valuation process and for the grid-related decision at issue. This stakeholder mapping is particularly important for decision- or action-oriented stakeholder engagement goals. Examples include regulatory oversight, implementation, operation, and maintenance.

- Note that technical or subject matter expertise may be sought throughout the valuation process. These technical experts may or may not be stakeholders. And, particularly in controversial or contentious situations, decisions about what technical expertise is sought (and from where) may serve either to avoid or stem controversy or to stir or amplify it.

\section{Determine the Role(s) Identified Stakeholders Play}

It is important to identify the "voices" or roles of identified stakeholders in the stakeholder engagement step, whether that activity is a single event or a series of interactions that occur over time. Specifying the role(s) that stakeholders will play may occur at the same time as one specifies stakeholder engagement goals and identifies which stakeholders to engage.

Stakeholders play many roles, ranging from passively receiving information to making decisions. From the standpoints of such matters as building or maintaining trust, clear communication, and effective interaction, it is important that involved parties have comparable expectations for the engagement. 
- Articulate role(s) for stakeholders-information recipients, information providers, decision makers, and so on.

- Consider the amount of time stakeholders may need to invest.

- Consider how stakeholders may benefit or what risks they may incur as a result of their engagement.

- Expect variation in responses, opinions, and so on. Plan for how to deal with that variation, especially when there are substantially different priorities or competing or conflicting objectives or opinions.

- Is consensus a goal of this stakeholder engagement? Is it necessary or desirable to reconcile differences? If so, how? Options include majority rule, Robert's Rules of Order, negotiation, and others.

- If stakeholder roles are consultative, is there a process for getting stakeholder feedback on (provisional) decisions made that are based on earlier stakeholder input?

- If stakeholder roles include decision making, do those stakeholders hold formal decisionmaking authority and/or have other fiduciary responsibility? If not, who does? And, what are the limits to stakeholder decision making?

- If a goal is to obtain buy-in through the stakeholder engagement process, consider the following:

- Whose buy-in is essential? Consider stakeholders within and outside any particular organization or entity.

- Why is the goal important or essential from their perspective(s)?

- What are the stakeholders' needs (perhaps defined in terms of their roles and responsibilities)? How do those needs influence the conditions for these parties' participation or buy-in?

\section{Determine the Mode(s) of Engagement}

Valuation-related stakeholder engagement likely will consist of multiple modes of engagement, so this activity focuses on how to construct that portfolio. There are nearly unlimited forms of engagement: faceto-face, print, online, social media; active or passive; broadcast or more narrowly cast; one-off, repeated (e.g., newsletters, tweets), or continuing (e.g., through panels or committees); and so on. Each option has pluses and minuses in terms of financial costs, individuals' time or convenience, extensiveness and representativeness, and degree to which it captures the desired degree of attention in an era of information overload. Making information available, either passively (e.g., on web sites) or actively (e.g., email or other notifications), does not ensure attention to or understanding of that information. Nor does it ensure stakeholder involvement or responsiveness.

Valuation analysts should consider a number of items as they develop their portfolio of stakeholder engagement modes, including the following:

- Ensure the modes of engagement are aligned with engagement goals.

○ The forms of engagement undertaken should be pragmatic for the stakeholders involved.

- Engagement likely will take multiple forms, ideally forms that reinforce one another and their collective ability to help achieve stakeholder engagement goals.

$\circ$ While there may be no single perfect form of engagement, some forms of engagement are illsuited for particular kinds of goals. For instance

- Creating a website may serve as a passive repository for updated information, but websites are ill-suited for forging or nurturing relationships or trust among stakeholders. 
- Online surveys technically are easy to produce and a relatively inexpensive way to reach many people scattered over space. But they can be plagued by low response rates (perhaps skewed by the types of individuals who do/don't respond) and are ill-suited for teasing out how positions may change in different circumstances or as a result of tradeoff discussions.

- Face-to-face workshops or discussions may be excellent for obtaining group feedback and nurturing relationships. But they may not represent what organizations/individuals can or will do, because of who is/isn't participating and because discussion may be dominated by particular personalities.

- Ensure the modes of engagement mesh with the schedule for the overall target activity.

- Engagement takes time, especially active, face-to-face engagement - time for planning, execution, and analysis.

- To the extent that results of stakeholder engagement feed into the particular steps or decision junctures, ensure the timing is appropriate. An obvious example is that stakeholder input on design criteria needs to get to the designers before designs largely are set.

This particular example is one in which iterative and interactive stakeholder engagement may be warranted. Designers may make incorrect assumptions about what stakeholders want or need, so early stakeholder input can help avoid problems. Similarly, there may be technical or regulatory constraints that prevent designers from using/achieving certain design criteria that are important to key stakeholders. Interactions with stakeholders may help to identify other alternatives that are technically sound, comply with rules and regulations, and meet stakeholder needs.

- Consider providing alternative means for eliciting information or feedback, such as providing opportunities for written comments. This strategy also can serve as a way to include stakeholders who want to be involved but were not invited to participate

\section{Conduct the Stakeholder Engagement}

Stakeholder engagement may take place several times, in many ways, over the course of the evaluation study. It is important to check regularly that (1) the original stakeholder engagement goals remain in effect and (2) the process has not drifted to some other implicit goal that might require different stakeholders or engagement methods. An example of the latter is a stakeholder engagement effort that begins by seeking to obtain informational input from varied stakeholders, then drifts over time toward seeking information about likely stakeholder decision making. Relevant stakeholders may vary between goals (though there could be overlap), as might the forms and substance of engagement. Analyses of stakeholder input may achieve neither the original stakeholder engagement goal nor the implicit, unstated goal.

- Revisit and, as necessary, refine stakeholder engagement goal(s), at least to ensure the goals are specific and relevant to the valuation step or the grid modernization decision-making phase. Modify the list of relevant stakeholders and engagement methods accordingly

- Gauge the extent to which the engagement process is on track to achieve the specified goal(s) - interim evaluation. Make refinements, as appropriate

- Document the substance of the engagement(s). Keep records of what stakeholders say, in their words as much as possible

- Note where there are disagreements, alternative perspectives, and so on. 
- It may be neither necessary nor appropriate to identify stakeholders by name or organization name.

- Separate the judgement of the analysts (those conducting the stakeholder engagement) from the stakeholder perspectives. Description of what stakeholders say is different from analysis of or judgments about what they say.

- Distinguish, as necessary, documentation that is intended for internal-to-valuation (or to grid decision-making) processes versus documentation that is intended to be a part of the public record.

An important issue to address is who convenes or directs the stakeholder engagement session. A PUC may lack the capacity or inclination to supervise a stakeholder process. If it is so inclined, the agency may be ill-equipped with the skills to lead a stakeholder process; and a facilitator can be useful but expensive. Also, a stakeholder process can benefit from an objective technical advisor who can insert important ideas and facts into the process to guide it in a productive way.

\section{Analyze the Results and Continue to Refine}

The nature of these analyses will vary according to the stakeholder engagement goals. Analyses can range from relatively informal descriptive characterizations to formalized, possibly quantitative characterizations of what stakeholders say. As each step in the process is taken, it is important to gauge the success of stakeholder engagement and, as appropriate, refine, articulate new goals, and restart/continue the stakeholder engagement process.

Appendix E includes some templates and a checklists and guidance that may be useful for this initial stakeholder engagement.

\section{Step 3 Deliverable}

- An initial stakeholder engagement plan that includes

0 the goals and objectives of stakeholder engagement

- a description of the identified relevant stakeholders

$\circ$ identified modes of engagement

- Records of the stakeholder engagement, including what stakeholders say, in their words as much as possible.

\section{Documentation of the Define Scope and Goal Phase}

At this point, the process should make sure documentation is completed for Phase A, Define Scope and Goal, of the valuation process. Each of the steps lists deliverables that include documentation of their respective steps. The appendices associated with the steps provide checklists or templates to assist in the development of the documentation.

\subsection{Phase B: Frame the Valuation Criteria}

The next two steps frame the required information (i.e., metrics) necessary to characterize and evaluate alternatives, decision criteria for the study, and the approach to integrate or consolidate the metrics to portray the valuation of each alternative. (Note that value is often a complex concept and may not be reducible to a single number or a quantitative tradeoff-relationship between metrics.)

\section{Step 4: Identify Relevant Impacts and Metrics}

The discussion of the previous phase was primarily based on documenting the context and identifying the alternatives to be examined in the valuation study. Step 4 further defines the study by identifying what impacts will be used to value each of the alternatives defined in Step 2, and the associated metrics that 
will be used to quantify those impacts. These potentially disparate metrics will subsequently be aggregated or synthesized into a meaningful whole.

When beginning the process of identifying what impacts will be characterized in a given valuation study, it is often useful to do a literature review of similar studies, or consult with experts, to identify what impacts have been used before. Once a set of commonly used impacts has been assembled, impacts can be added or removed from the set based on the unique circumstances of the current valuation study. The following are questions to help a practitioner think about whether to add or remove impacts:

- Do you lack either the data or modeling capabilities necessary to quantify a given impact? If so, you may have to exclude it. However, if the metric is important you may still need to address it, perhaps as an uncertainty in Step 6. Conversely, do you have data about your system that may not have been available to previous analysts that would enable a neglected impact to be studied?

- Are the perspectives of the previous studies and your proposed study the same? For example, is your study from the societal perspective, and previous studies from the ratepayer perspective? If so, you may need to subtract or add impacts depending on where the boundaries are being drawn.

- Are there irrelevant impacts in previous studies? For example, a study done in New York State may have an impact on costs of emission allowances that is a product of local policy and would not be relevant to a study in a different state.

In determining what impacts should be characterized in a particular valuation study, it may be helpful to think of the system being studied. The alternatives being studied will presumably impact a variety of system properties - they will change the state of the system. Generation types and locations may be different, transmission or other asset topologies may change, demands may be higher or lower or follow different patterns. Identifying all the system properties that may change as the result of an alternative may help identify what impact categories should be included in the valuation study.

A GMLC Foundational Metrics project has developed a catalog of the main energy- and power systemrelated properties and metrics that may be useful in this exercise. Each of the cataloged metrics relates to an impact that could be considered in a valuation study. Only a subset of those impacts will be applicable to a specific valuation effort, but the catalog may be helpful in identifying which ones to consider. For some valuation efforts, industry guides may exist and may provide useful references (e.g., the Electric Power Research Institute's Integrated Grid). There may also be other non-power system metrics, such as economic indicators, that will influence the decision. These could require economic models to complete the valuation analysis.

Ideally, all of the relevant system properties that could be affected by the alternatives being considered should be identified and, for each system property, one or more impacts that communicate the state of that property should be selected. In practice, there is a long tail of increasingly negligible system properties that will be affected, and it would be impractical to try to identify them all, let alone characterize them. There is no generalizable rule about how small an impact can justifiably be considered negligible.

Certain exercises, such as soliciting stakeholder opinions, can help to identify which impacts should ultimately be studied. Figure 2 shows the results of such an exercise from the TVA distributed generation - integrated value (DG-IV) study, in which stakeholders were asked to evaluate which types of values were appropriate to include in the quantitative methodology. A survey was taken of each stakeholder on the review team for their estimate of the applicability and quantifiability of various potential values to be examined. Formal strategies for eliciting stakeholders' opinions about which metrics should be quantified include stakeholder surveys, polls, Delphi, review boards, or other techniques. 


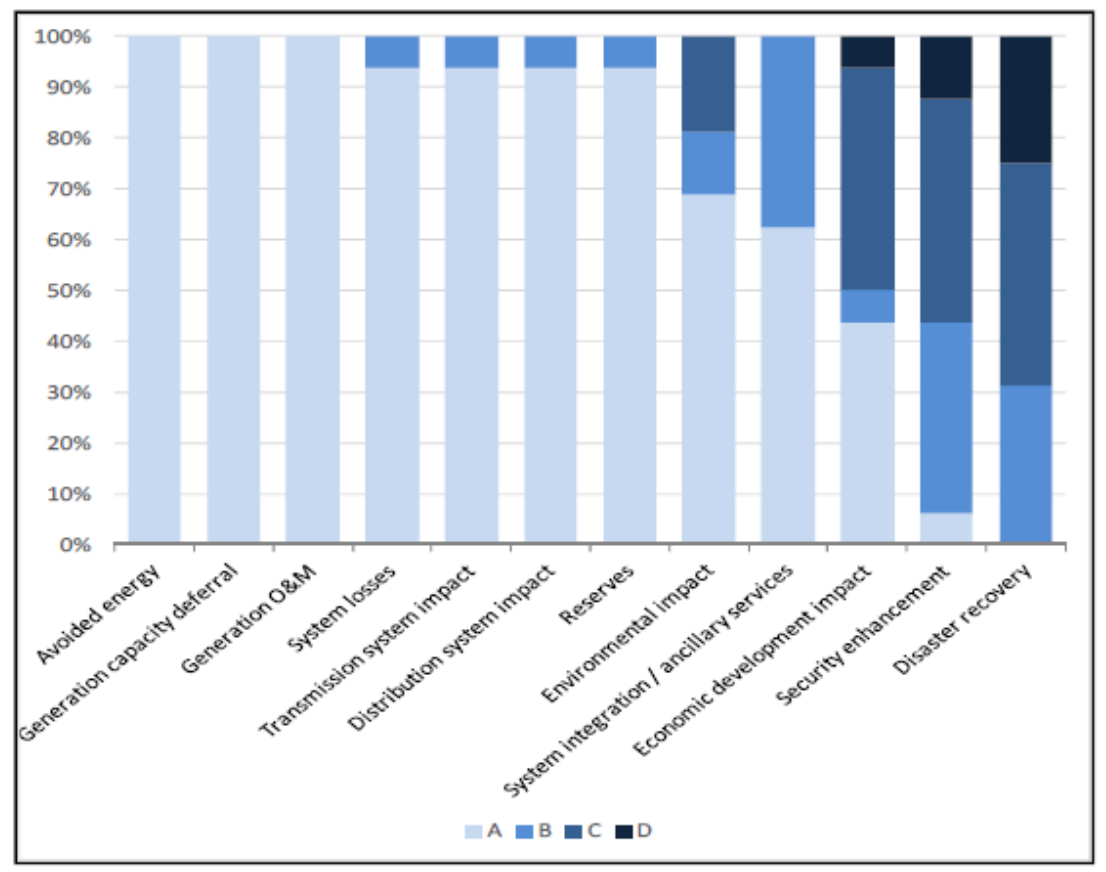

D - Not applicable

C - Applicable, belongs in

public policy discussions

rather than analytic

framework

B - Applicable, not

currently quantifiable,

placeholder for future

consideration

A - Applicable, currently quantifiable, belongs in

this analytic framework

Figure 2. Example of stakeholder perspectives on components of TVA's DG-IV calculation methodology (TVA 2015a)

Finally, as part of identifying which impacts will be studied, the units of measurement for describing the impacts should also be specified. For example, one of the impacts of a decision to invest in new distribution would be a change in reliability compared with the status quo without the investment. One way to measure the change in reliability is a metric like the System Average Interruption Duration Index (SAIDI), which indicates the average duration of outage experienced per customer served by the utility. The change in SAIDI with and without the distribution investment would measure the impact of the decision on reliability. Each impact will need a corresponding metric (or multiple metrics).

\section{Stakeholder Considerations}

In Step 4, stakeholders primarily help to (1) articulate the impacts associated with the grid modernization issue in question that are most relevant to them and (2) establish priorities among the multiple impacts identified that will become the focus of the valuation analyses. Stakeholders primarily help to identify which subset of impacts, of all the potential impacts identified, on which the valuation will focus. This subset of impacts will be quantified through the valuation process.

\section{Key Potential Challenges}

As in Steps 1 and 2, two major challenges associated with Step 4 are to ensure that there are clear expectations among all involved regarding what role stakeholders will play in making Step 4 decisions and how disagreements will be resolved. It would not be surprising for stakeholders to suggest more impacts, and which impacts will count in the valuation analyses, and to disagree about which impacts matter most. There are multiple ways to make these decisions. Each approach has advantages and disadvantages. As one example, it may be helpful to establish a priori or in consultation with involved stakeholders the decision-making criteria, perhaps separated into categories such as required and desired. But these decisions can suffer from the same potential problems as discussed for Steps 1 and 2. Another option is for the valuation analysts to select a workable set of impacts on which to focus (from the standpoint of the valuation analysis). Documenting the reasons for choosing to focus on a subset of metrics is essential; if the analysts' rationale is not clearly expressed to the participating stakeholders, or 
if stakeholders expected to have more of a say in determining which impacts on which to focus, this approach can become a point of contention that undermines trust between valuation analysts and stakeholders.

Another example of an approach to use is to survey stakeholders about their perspectives, as illustrated in Figure 2. This approach has the advantage of tapping participants' input and evaluating it in a readily comparable way. However, surveys may not identify all of the impacts or metrics that stakeholders would suggest on their own and may not distinguish among priorities (e.g., a respondent's second-highest priority may be nearly as important as the highest priority or not at all important). And survey data do not automatically determine how subsequent choices should be made (e.g., most responses, average responses).

The generic stakeholder engagement guide suggests the importance of identifying metrics when articulating goals. From this standpoint, valuation analysis impacts should be identified initially during Step 1 and refined during Step 4. Regardless, the impacts and metrics identified during Step 4 may call into question the choices made in association with Steps 2 and 3 and prompt their refinement.

There are multiple possible ways in which to elicit input from stakeholders about which impacts to quantify through the valuation process - stakeholder surveys, polls, Delphi, review boards, or other techniques. These illustrative techniques are differently suited for resolving disagreements among stakeholders. For example, traditional Delphi methods often seek consensus among the participating experts, and review boards can be charged with producing a consensus. In contrast, surveys and polls are not oriented toward achieving consensus. Analysts select options for how to represent and use findings, such as averaging responses on surveys, where " 3 " can result either from " 3 " being the dominant response or from roughly equal " 1 " and " 5 " answers. Or, especially in polls with many options and where the votes are split across those options, the option getting the most votes still may be unacceptable to most of the respondents.

Although, as stated earlier, "greater emphasis should generally be placed on quantifying impacts that are of highest priority to the decision maker," doing so may not be straightforward in situations where there is not a singular decision maker. Some grid modernization decision making requires go/no-go decisions by multiple parties, perhaps with the additional need for approval by a public service commission. These multiple decision makers may identify a number of different highest-priority impacts that, collectively, the valuation analyses may not be able to accommodate because of budgetary constraints or other reasons. Thus, additional potential challenges are to identify the set of high-priority impacts for key decision makers and choose which of those impacts to include rather than exclude in the valuation analysis.

\section{Additional Resources}

Appendix F includes checklists of potential metrics by category that may be useful for accomplishing Step 4. It is drawn from work performed by the Foundational GMLC project on Metrics (Anderson et al. 2017), which has developed tables of potential properties and metrics for each of the major properties of valuation. Many of these may be inapplicable to the purpose of the study; the evaluation team may select a subset of relevant metrics.

\section{Step 4 Deliverable}

- The key set of impacts and metrics with the units of measurement to be used

- A list of non-quantifiable impacts that may be considered by the decision maker

- A list and justification for those impacts that have been identified but will not be considered during the valuation 


\section{Step 5: Determine Multi-Criteria Integration Approach}

At this point, the process will have generated a list of alternatives to study and a list of metrics to quantify the state of key system properties under those alternatives. Each of the system properties will be characterized by metrics that may differ from one another. For a valuation effort to be useful, it must ultimately present these potentially disparate metrics in a way that can be understood, be compared against the results of other studies, and facilitate subsequent decision making.

It is useful to decide how the results will be integrated and communicated at this point, as that choice may influence subsequent decisions. Additionally, a decision about how to communicate the results may prompt rethinking which metrics are a priority or should be quantified. For example, it may be practical to downplay a system property that is non-quantifiable if the valuation effort is to inform an action that can only handle quantifiable (e.g., monetizable) results. Two possible approaches are described below:

\section{Integration Approach 1: List of Impacts for Each Alternative in a Table or Graphic}

In many situations, it may be sufficient for a valuation effort to simply state the estimated impacts of all key impact metrics for each alternative, without seeking to aggregate them into a single value. This approach may be appropriate if, for example, the valuation is meant to support a decision by decision makers who are not formally part of the valuation process. For example, a legislature may request a valuation study of a particular policy intervention that would have monetary, health, and equity impacts. Although it is technically possible to monetize the intervention impacts or use decision science to produce a single-metric result, it might be preferable to simply present the legislative body with the impact in each area and let it determine how to interpret and weigh the disparate metrics, using its own methodologies.

Metrics for different impacts will likely have different units (e.g., dollars, jobs, land use), in which case, metrics could be listed with their associated units. Some metrics may concern whether a certain constraint is met, in which case the value for that metric is "yes" or "no". In other cases, the actual quantity may be difficult to express, so for clarity the value may be shown as "low," "medium," or "high." There are many ways to characterize and describe impacts without strict quantification. The impacts can be communicated in a table or graphic.

Table 3 gives a very simple example of different impacts that may be generated for three alternatives. The table simply lists the impacts and their metrics. In Figure 3, a spider diagram presents the quantitative impacts. While it shows the relative standing of the different alternatives for each impact, the impacts should not be cross-compared. Rather the diagram serves only to assist stakeholders in visually identifying the relative impacts of each alternative in a compact form.

Table 3. Example of impacts from different alternatives

\begin{tabular}{|l|c|c|c|}
\hline & Alt. A & Alt. B & Alt. C \\
\hline Cost (M\$) & 20 & 15 & 10 \\
\hline Profits (\%) & 12 & 11 & 10 \\
\hline Emissions (ton) & 20 & 15 & 10 \\
\hline Land (acres) & 200 & 100 & 300 \\
\hline$\Delta$ losses (\%) & 3 & 2 & 3 \\
\hline Jobs (x) & 200 & 500 & 400 \\
\hline Vista impact & low & high & med \\
\hline Technology & new & exist & mature \\
\hline
\end{tabular}




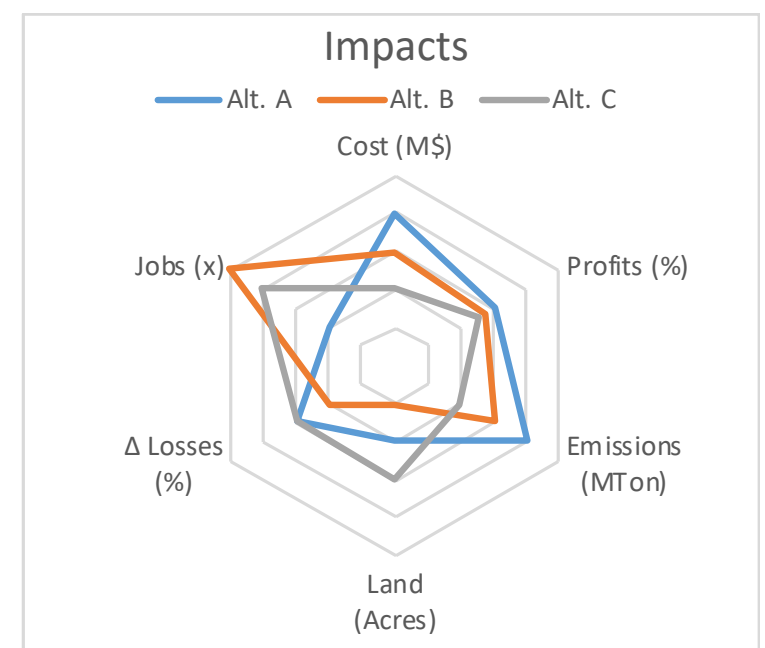

Figure 3. Graphing of quantitative factors from example.

The presentation format of Figure 3 could also be used to present multiple metrics that have the same measurement units. For example, air pollutant emissions from different alternatives may include $\mathrm{CO}, \mathrm{SO}_{2}$, NOx, hydrocarbons, particulates, and heavy metals. These may be expressed in micrograms per cubic meter $\left(\mu \mathrm{g} / \mathrm{m}_{3}\right)$, parts per million, or parts per billion. The spider diagram of Figure 2 could show these six quantities and indicate the maximum allowable levels of each (as set by federal or state government regulations). Figure 4 gives an example of a different way to visualize multiple values. In 2011 the EIPC modeled 8 different alternatives (futures) under a variety of sensitivities totaling more than 87 scenarios (EIPC 2011). Many impacts were quantified in the analysis, and tables were generated presenting those values. However, the quantity of data could not be easily examined in a single table. One method of communicating results was to pair different impacts in an X-Y graph for all 87 scenarios. This brought out a clustering of results that created insights into the overall result. It assisted in the selection of three alternatives to be further analyzed in later stages of the transmission study. 
2030 Max Inter-Region Flow during Peak (GW) vs 2030 US Electric CO2 Emissions Reductions from 2005 (\%)

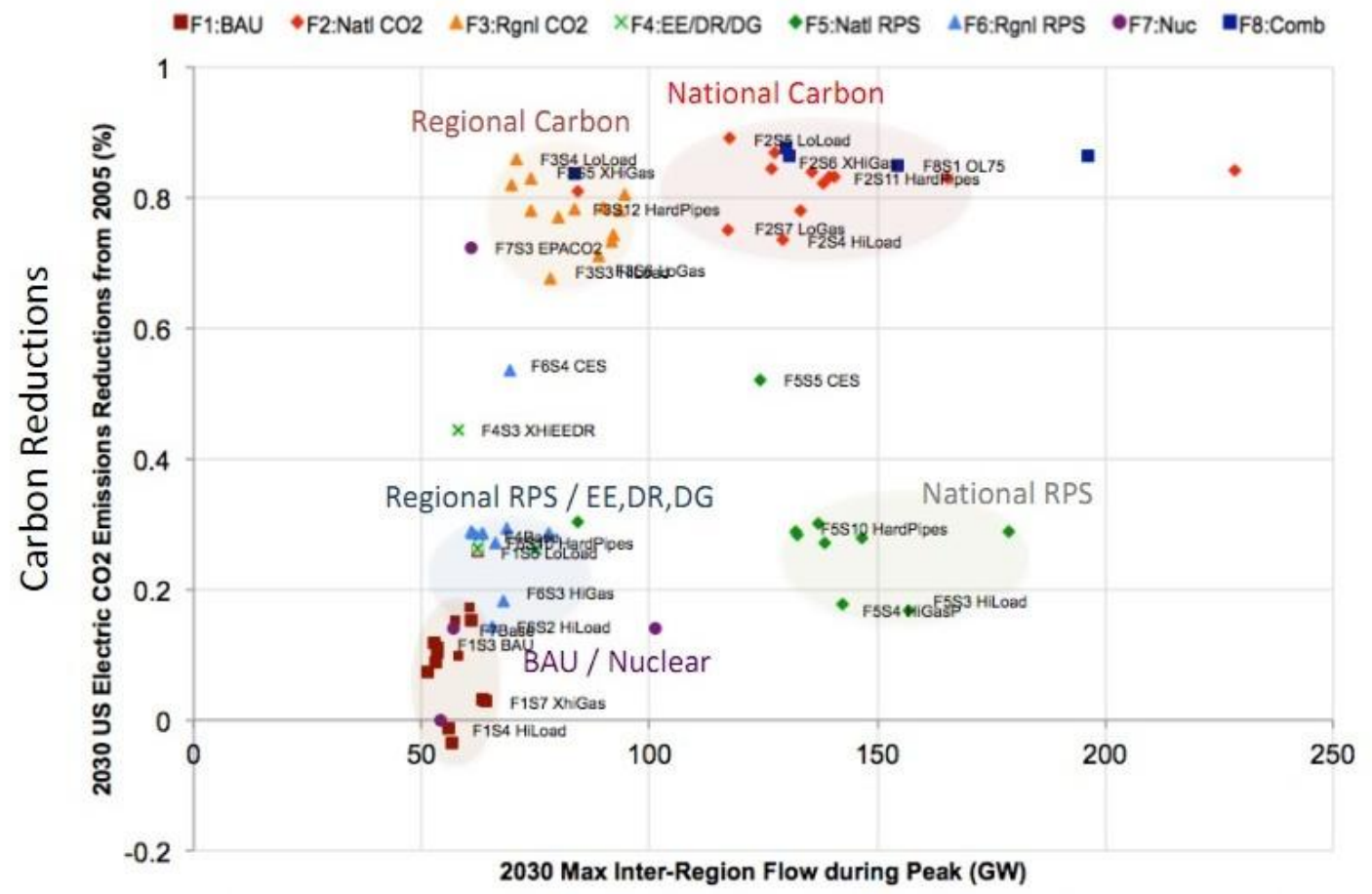

Figure 4. Cross comparison of two impact metrics from the EIPC transmission study. (EIPC 2011)

In cases where there are a large number of metrics and/or cases, tables can be enhanced by the use of heat maps, in which cells in a table are colored based on each alternative's value for each metric. In the EIPC transmission study, there were more than 31 key metrics, with some further disaggregated by regions or technologies. In Table 4, 4 of the key metrics from 28 of the scenarios analyzed (representing all of the cases from 2 of the 8 futures) are shown. Each metric is colored so that the lowest value of all cases is dark red, the highest value is dark blue, and the average is yellow. This approach allows a reader to rapidly identify the trends and outliers. Relationships between different metrics can be readily discerned.

In cases where the valuation is meant to support a decision that is primarily motivated by the desire for economic efficiency, and all impacts can be valued monetarily, standard cost-benefit analysis (CBA) can be used to summarize each alternative's impacts in terms of a single net dollar benefit or ratio of costs to benefits. Monetization of all impacts provides a single unit that can be used to combine all impactstypically dollars discounted back to a reference year. Monetization indicates which of the alternatives will result in the greatest overall welfare. It does not, however, capture distribution of benefits among the affected stakeholders. Traditional CBA approaches include financial CBA, in which only impacts with direct market values are considered, and social CBA, wherein economic valuation techniques can be used to monetize nonmarket impacts. Examples of the latter include the social cost of carbon (which places a dollar value on present-day greenhouse gas emissions) and value of statistical life techniques used to monetize the impacts of power system externalities on human health. 
Table 4. Heat map table of some of the metrics from the EIPC transmission study. (EIPC 2011)

\begin{tabular}{|c|c|c|c|c|}
\hline Policy drivers/goals & $\begin{array}{c}2030 \text { max } \\
\text { inter-region } \\
\text { flow during } \\
\text { peak }\end{array}$ & $\begin{array}{c}2030 \text { max } \\
\text { all-hour } \\
\text { inter-region } \\
\text { flow }\end{array}$ & $\begin{array}{c}2030 \mathrm{EI} \\
\text { total } \\
\text { transferred } \\
\text { energy }\end{array}$ & $\begin{array}{l}\text { El 2015- } \\
2030 \mathrm{CO}_{2} \\
\text { emissions }\end{array}$ \\
\hline & $(G W)$ & $(G W)$ & (TWh) & (MMT) \\
\hline Final BAU & 53 & 115 & 296 & 26,031 \\
\hline BAU with Hardened Limits & 53 & 115 & 297 & 26,012 \\
\hline BAU, High Load Growth & 56 & 112 & 372 & 28,608 \\
\hline BAU, Low Load Growth & 62 & 113 & 309 & 23,193 \\
\hline BAU, High Gas Prices & 63 & 119 & 454 & 29,503 \\
\hline BAU, Extra High Gas Prices & 64 & 118 & 455 & 29,857 \\
\hline BAU, Extra Low Renewable Resources Cost & 60 & 113 & 386 & 25,488 \\
\hline BAU, Increased EE/DR and RPS Requirements & 61 & 118 & 292 & 25,235 \\
\hline BAU, Higher PHEV & 54 & 115 & 319 & 26,151 \\
\hline BAU, Decreased Renewable Resources Cost & 57 & 108 & 351 & 25,901 \\
\hline BAU, Delayed EPA Regulations (+20 years) & 53 & 107 & 321 & 27,218 \\
\hline BAU, Reduced EE/DR and RPS Requirements & 51 & 102 & 357 & 26,939 \\
\hline BAU, Less Aggressive EPA Regulations ( +5 years) & 53 & 115 & 296 & 26,586 \\
\hline BAU, PTC/ITC Expire and RPS Removed & 58 & 113 & 309 & 26,496 \\
\hline BAU, PTC/ITC Expire, RPS Removed, High Load & 57 & 108 & 366 & 29,094 \\
\hline National Carbon Constraint & 84 & 124 & 542 & 11,004 \\
\hline National Carbon, Soft Constraint, $75 \%$ Overload & 166 & 266 & 886 & 10,610 \\
\hline National Carbon, Soft Trans., 25\% Overload & 229 & 351 & 1,368 & 10,105 \\
\hline National Carbon with Reduced Friction Charges & 141 & 180 & 905 & 10,301 \\
\hline National Carbon with High Load Growth & 129 & 179 & 793 & 12,411 \\
\hline National Carbon with Low Load Growth & 118 & 165 & 770 & 9,215 \\
\hline National Carbon with Extra High Gas Prices & 127 & 171 & 932 & 13,669 \\
\hline National Carbon with Low Gas Prices & 117 & 168 & 760 & 10,461 \\
\hline National Carbon with Flat Carbon Price post 2030 & 138 & 180 & 863 & 10,163 \\
\hline National Carbon with Lower Carbon Costs & 133 & 182 & 798 & 11,898 \\
\hline National Carbon with Extra Low Renewable Costs & 127 & 177 & 913 & 9,609 \\
\hline National Carbon Base with Hardened Tx Limits & 139 & 175 & 853 & 10,403 \\
\hline National Carbon with Increased Variable Limits & 136 & 179 & 872 & 10,428 \\
\hline
\end{tabular}

BAU $=$ business as usual

\section{Integration Approach 2: Monetization in a Cost-Benefit Analysis Framework}

The preceding paragraph describes a situation in which each alternatives' costs and benefits are estimated and monetized as part of a valuation, with the objective of providing a net benefit or ratio of benefits. In some situations, it may be more appropriate to perform a valuation from a particular stakeholder's perspective, in which certain impacts are excluded because they would not be borne by that stakeholder. For example, a value-of-solar study may seek to quantify only the net benefit that distributed solar provides to the grid, without including the cost of installing a system. This makes sense if the valuation is meant to inform a decision about how to compensate distributed solar, where the costs of making the investment are borne by solar adopters and may not be material to the decision at hand. Another example is an independent power producer who may be most concerned with the financial profitability of a project, while the grid owner as a stakeholder would be more concerned with the impact of the project on the grid. 
If the valuation is meant to support decisions where the distribution of benefits is also of concern, then the single monetized values of each alternative may be supplemented with additional information that shows the benefits obtained by sub-populations. This may be most useful if there are opportunities to control contributions to costs (or other transfers of wealth) by those sub-populations when an alternative is implemented. For example, a utility's valuation of a microgrid project may reveal unequal gains across its ratepayers, which may guide decisions about how various sub-populations would contribute to the project's costs. ${ }^{6}$

When only some impacts can be monetized, an alternative is to use techniques such as multi-attribute decision making that helps develop weighting for the different impacts. There are multiple approaches to generating these weighting factors, from soliciting input from stakeholders to more quantitative methods that transform impacts recorded in disparate units into a single measure of stakeholder utility.

While such methods may lead to different results for different stakeholders, the reasons for the differences can be made more transparent and subject to negotiation. Different technologies will have strengths or weaknesses in different metrics, and different stakeholders will place different weights on the different metrics. Note that, even in cases where disparate impacts are aggregated into a single metric, it is a best practice to document the individual impacts and make information available to anyone interpreting the results of the valuation study. ${ }^{7}$

The integration of the different metrics should not inadvertently overweight or double-count certain impacts over others. For example, if cost impacts are included through multiple metrics (price, total cost, cost per $\mathrm{kWh}$ ) that are tied to one or two key inputs (fuel price, capital cost) but other impacts (environment, security, resilience) are only measured through a few metrics or are neglected, then cost may inadvertently become the main driver of a decision. Similarly, overweighting environmental or reliability impacts may bias the overall value to their metrics. Double-counting can sometimes occur by applying a savings as both an increase in benefits and a reduction in costs. The relative weighting of impacts is often where key disagreements in value occur, so due deliberation is needed on how results will be integrated and reported.

\section{Stakeholder Considerations}

Because stakeholders often have competing objectives (e.g., lowest energy cost versus lowest environmental impacts), the trade-offs among metrics may be the most contentious issues in policy debates. While stakeholders may play limited or no direct roles in shaping Step 5, analysts must incorporate (or anticipate) stakeholder input in determining which integration approach to use. Thus, while stakeholders will receive information from analysts describing which approach is being taken and the reasons for that choice, it is prudent for valuation analysts to engage those stakeholders who also are key decision makers to ensure that the integration approach selected actually meets these decision makers' needs. Additionally, analysts should engage stakeholders to ensure that approaches that weight

\footnotetext{
${ }^{6}$ Allocation of costs and benefits is a policy decision often made in conjunction with the selection of alternatives. For example, energy efficiency measures may reduce the need for new generation; generators are included in a utility's rate base, but energy efficiency investments may not be. Once an alternative with the greatest overall value has been selected, PUCs usually determine how to allocate the costs and benefits associated with the increased value through tariff design and conditions on rate base inclusion.

${ }^{7}$ Even when all metrics are monetized, there is often a need to also present each metric's value-Integration Approach 1. For example, stakeholders may disagree on how to set the social cost of carbon. While the overall value can be monetized, presenting the individual metrics enables examination of how overall value is sensitive to assumptions about quantifying the social cost of carbon or other externalities.
} 
attributes according to stakeholder perspectives accurately reflect those stakeholders' (and potential key interveners') perspectives about what the weights should be.

\section{Step 5 Deliverable}

An analysis requirements document identifying and prioritizing the metrics to be used for decision making and stating the multi-criteria integration approach. This must include explicit documentation of how impacts will be integrated into values and presented to inform decision makers and to objectively inform policy debates with intervening or competing stakeholders. If measurement units will be transformed into other units to support decision making (e.g., monetization approaches, utility functions), the transformation methods should also be noted for the valuation process to be transparent.

\section{Documentation of the Valuation Criteria Phase}

At this point, the process should make sure documentation is completed for the valuation criteria phase of the valuation process. Each of the steps lists deliverables that include documentation of their respective steps. The appendices associated with the steps provide checklists or templates to assist in the development of the documentation. It would be worthwhile for the decision maker and analysis team to review whether the information from Steps 4 and 5 necessitate an iteration to update or modify the stated purpose, alternatives considered, or list of stakeholders from the previous phase.

\subsection{Phase C: Design the Analysis}

The three steps in this phase construct the study, including the methods, tools, assumptions, and input data to be used. This includes determining the methods of considering uncertainty in the analysis and consolidated impacts from the different alternatives.

\section{Step 6: Determine Approach to Address Uncertainty}

\section{Context}

Valuation can be more art than science, given the various techniques available to calculate the value of new technology. Recall from previous sections that a valuation is essentially the process of estimating a "price" in the market place. Valuation estimates are affected by a range of uncertainties. Uncertainty exists with regard to the current state of the world, resource availability and prices, the imperfect ability to measure impacts of alternatives, imperfect tools, lack of information or data, and selection of inputs. Uncertainty is a real and universal occurrence in valuation. It is not an insurmountable barrier, as sources of uncertainty are rational and can be identified. Identification and description of uncertainty as part of a valuation process strengthens the transparency and credibility of the valuation outcome.

\section{Documentation of Uncertainty}

Documenting uncertainties can aid in prioritizing uncertainties, identifying strategies to manage uncertainty, and increasing the transparency of valuation. The DoD Risk Management Guide for DoD Acquisition, sixth edition, provides a disciplined environment for proactive decision making to identify and mitigate risks. Taking the appropriate parts of the DoD approach (and substituting "uncertainty" for "risk") gives the following results:

1. Identify uncertainties (what is uncertain and what is uncertain about it)

2. Assess uncertainties

A. Magnitude of uncertainty

B. Impact of uncertainty on evaluation metrics and conclusion

C. Impact of uncertainty on valuation conclusion 
3. Select uncertainty mitigation methods

This analysis can be summarized in an uncertainty risk management table (Table 5).

Table 5. Uncertainty risk management table.

\begin{tabular}{|l|l|l|l|l|l|}
\hline Uncertainty & $\begin{array}{c}\text { Type of } \\
\text { uncertainty }\end{array}$ & $\begin{array}{c}\text { Magnitude of } \\
\text { uncertainty }\end{array}$ & $\begin{array}{c}\text { Impact of } \\
\text { uncertainty } \\
\text { (low/med/high) }\end{array}$ & $\begin{array}{c}\text { Method(s) to } \\
\text { address } \\
\text { uncertainty }\end{array}$ & $\begin{array}{c}\text { Comments/ } \\
\text { reasoning }\end{array}$ \\
\hline & & & & & \\
\hline & & & & & \\
\hline
\end{tabular}

Each uncertainty identified in the valuation process (each row in the summary table) would be explained in detail in text, including the choices made regarding how to model or otherwise address the uncertainty.

This approach results in a clear enumeration of identified uncertainties and a uniform format to describe how the uncertainty was assessed and addressed. The table, particularly the column labeled "Impact of uncertainty," also provides a clear way to map key metrics in the analysis to uncertainties that may impact the results of the analysis.

\section{Prioritization of Resources to Reduce or Manage Uncertainty}

The uncertainty table summarizes the various sources of uncertainty, the magnitude of the uncertainty, and the impact of the uncertainty. Uncertainties that are large and impactful deserve prioritization in the analysis. Resources available to conduct the analysis can be allocated to reduce the magnitude of the uncertainty (i.e., getting better data) or to identify tools to incorporate the uncertainty into the analysis. Strategies for managing uncertainty will be addressed in the following section. Here, we discuss strategies for reducing the magnitude of the uncertainty.

In some cases, uncertainty in parameters can be reduced through the use of improved data. The cost of improving data should be compared with the costs of managing the uncertainty in the analysis. One example of uncertain data that can be addressed through acquiring improved data is when analysts have data from studies conducted in another region, but conditions are not exactly the same in the region of interest. In this case, analysts could recreate the study for the local region. Pilot studies, for example, can be executed to develop better estimates for the local region.

Another strategy for improving data is expert elicitation. Expert elicitations yield a collection of informed projections of parameters whose mean value can be more accurate than a single parameter. Expert elicitations also yield information about the degree of agreement or disagreement between experts, which can also be helpful in characterizing the remaining uncertainty that needs to be managed through the analysis.

\section{Strategies for Managing Uncertainty in Analysis}

Planners in the electricity sector regularly need to manage uncertainty in comparing options. Common techniques for managing uncertainty include sensitivity analysis, scenario analysis, and probabilistic analysis (Hirst and Schweitzer 1989; Wilkerson et al. 2014; Kahrl et al. 2016). Descriptions of those techniques in the context of IRP are provided in Table 6. 
Table 6. Techniques to manage uncertainty in valuation (adapted from Wilkerson et al. 2014).

\begin{tabular}{ll}
\hline Technique & Description \\
\hline Sensitivity analysis & $\begin{array}{l}\text { Key factors of candidate resource plans and portfolios are varied to see how they } \\
\text { respond to these variations }\end{array}$ \\
\hline Scenario analysis & $\begin{array}{l}\text { Alternative visions of the future are developed, appropriate combinations of resources } \\
\text { are identified that best fit each future, and the best options are combined into a unified } \\
\text { plan }\end{array}$ \\
\hline Probabilistic analysis & $\begin{array}{l}\text { Probabilities are assigned to different values of key uncertain variables (possibly } \\
\text { identified through sensitivity analysis). Outcomes are identified that are associated } \\
\text { with different values of the key factors in combination. Results often include the } \\
\text { expected outcome and probability distribution for those key factors (e.g., natural gas } \\
\text { price) }\end{array}$ \\
\hline
\end{tabular}

There are two dimensions of uncertainty to consider in valuation. Both are challenges in valuation, but the strategies for managing the uncertainty can be different between the two. The first is that estimates used in modeling may be systematically different from the true value (i.e., biased) This bias can occur for a variety of reasons, including modeling errors, data or assumptions that might have been accurate at one time but are now outdated, or aspects that fall outside of the scope of the analysis. The second is that data may be imprecise or random: parameters may change significantly, leading to different values being observed depending on when a measurement occurs. For example, a $100 \mathrm{MW}$ generator with a forced outage rate of $5 \%$ will have an availability of $0 \mathrm{MW}$ or $100 \mathrm{MW}$ depending on when its availability is measured, even if the true expected value of its availability is $95 \mathrm{MW}$.

\section{Managing systematic bias}

Inputs to valuation studies can have bias simply because the cost of improving estimates is too high to justify the resources needed to maximize accuracy. In this case, the primary strategy to manage the uncertainty is determining if the decisions are robust to a plausible range of uncertain parameters. This robustness analysis can be done through sensitivity analysis, in which the effect of varying one uncertain parameter at a time is analyzed, or scenario analysis, in which the effect of multiple parameters moving in conjunction with one another is analyzed. Each is described in greater detail in the following paragraphs. Demonstrating that the relative attractiveness of alternatives is robust to reasonable ranges of uncertain parameters acknowledges the uncertainty, while at the same time providing comfort that the results of the analysis are not dependent on the particular assumptions or data used in the analysis. The converse can also be true, however: if the robustness analysis finds that the relative attractiveness of alternatives changes when parameters are varied within a plausible range, then more resources may be required to reduce uncertainty before making a decision. In practice, the extent to which uncertainties can be explored in a robustness analysis is dependent upon available resources. Decision makers and analysts should prioritize those uncertainties that are likely to materially influence those impacts and metrics of great concern to them or to stakeholders.

The following are further details regarding sensitivity analysis and scenario planning techniques.

- Sensitivity analysis is the practice of adjusting a single input to a model to characterize how sensitive the various results are to that particular input. Typically, an analyst would explore both positive and negative adjustments for all the inputs whose value uncertainty is expected to have a non-trivial impact on the valuation results. Sensitivity analysis is used to evaluate the robustness of the quantified values - and ultimately the relative comparison between alternatives - against a range of values for one or more key parameters. An example might include exploring the sensitivity of capacity expansion simulations to a range of input costs for a technology (e.g. varying the cost of solar or wind resource), or fossil fuel prices. This is useful because the 
distribution representing the uncertainty of many of these parameters is unknown and is arguably unknowable in many cases.

- Scenario planning involves constructing multiple combinations of input parameters and assumptions ("scenarios") to explore the sensitivity of valuation to different states of the world. The line between sensitivity analysis and scenario planning is not necessarily bright. Instead, where sensitivity analyses generally explore the influence of a range of parameter values, scenario planning contains an element of story-telling, focusing on constructing a variety of plausible future outcomes. Scenario planning is useful to bookend modeled outcomes by exploring a range of futures. Stakeholder engagement in the scenario definition process is important to explore a full range of scenarios and ensure that practical concerns can be expressed (as possible) in modeled outcomes.

\section{Managing inherently random data}

Even if the parameters are not biased, there is still the issue that some data are imprecise or random. Natural gas prices, for example, can be both biased (users might under- or over-forecast the mean value over the next few years) and imprecise (natural gas prices vary considerably around their mean value because of various constraints in the supply chain or demand shocks). Imprecise or random data can be managed through probabilistic analysis.

A common strategy for managing the uncertainty related to imprecision is to conduct analysis based on expected values. For example, even if we know that the natural gas price is likely to vary around its seasonal average value by $\pm \$ 3 / \mathrm{MMBtu}$, expected value analysis would conduct the analysis with the single expected value of the natural gas price for the next year. Expected value analysis is an especially powerful tool when systems are linear.

When systems are nonlinear, however, there is no guarantee that the output of an analysis based only on the expected value of inputs will be equivalent to the expected value accounting for the full distribution of the imprecise inputs. As an example, Figure 5 illustrates a hypothetical distribution of natural gas prices around an expected value of the natural gas price, along with a total system cost that is either linear with natural gas price (blue line) or nonlinear with natural gas price (red line). In the case where the cost is linear with the natural gas price, the expected value of the cost is equivalent whether the full distribution of natural gas prices is directly accounted for in the calculation (blue square), or the cost is found by simply evaluating the system cost at the expected value of the natural gas price (i.e., the intersection of the gray dashed line and the blue line). In the case where the system cost is nonlinear with natural gas price, however, the expected value of the cost considering the distribution of possible natural gas prices (red dot) is not equivalent to the system cost based on the expected value of the natural gas price (point where the gray dashed line intersects the red line).

In this particular example, the nonlinearity arises from the dispatch of natural gas plants changing in response to changes in the price of natural gas. If the price of natural gas is low, then more natural gas plants will be dispatched for longer periods. When the natural gas price is high, on the other hand, the natural gas plants will be dispatched as little as possible. If the natural gas price varies randomly throughout the analysis period, then estimating the system cost using only the expected value of the natural gas price will not lead to the true expected value of the system cost. 


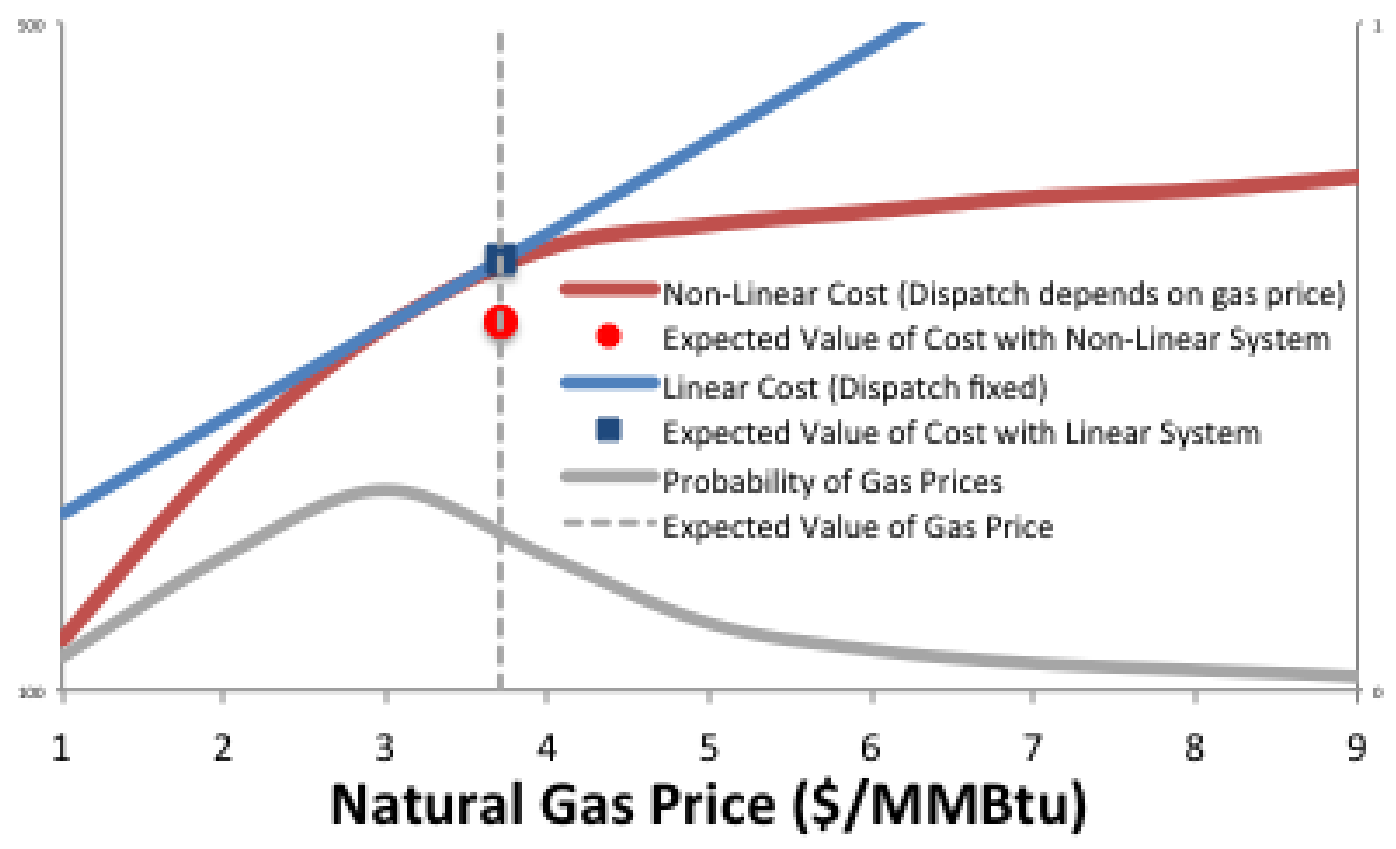

Figure 5. Illustration showing case where using expected value of inputs does not lead to true expected value of outputs for nonlinear systems.

Whereas linear systems can manage imprecise data through expected value analysis, managing uncertainty in cases with nonlinear systems and imprecise data requires evaluating the full distribution of the random data. As many aspects of the power system exhibit randomness, many modeling tools have the ability to estimate the true expected value of nonlinear systems through evaluation of the distribution of the random data. Techniques include running simulations over a full year or longer of hourly data; directly convolving the distributions of random parameters; or, more commonly, using Monte Carlo simulation.

In Monte Carlo simulation, random samples of input parameters are selected to generate hundreds to thousands of different combinations. A large number of samples may be necessary to fully explore uncertainty in a statistical way, so stochastic methods may exceed the computational resources practically available to an analysis team. These methods themselves also may introduce additional layers of uncertainty in the methods used to model and sample input parameters. This is because both the input and hence the model output distributions may be inherently uncertain, and care must be taken that this method does not provide the false illusion of having modeled uncertainty accurately.

Using Market Simulations for Economic Assessment of Transmission Upgrades: Application of the California ISO Approach

One of the five principles in the transmission planning approach developed by the California ISO is "explicit uncertainty analysis." Uncertainty complicates evaluation of the decision of whether or not to build transmission, including uncertainty around load growth, fuel costs, future generation capacity, exercise of market power, and availability of hydropower. Some of these risks and uncertainties are readily quantified, but others are not.

The transmission planning methodology calls for managing this uncertainty through scenario analysis or probabilistic analysis. It recommends reporting analysis results based on expected conditions as well as on unusual but plausible situations. The various measures are then reported individually to provide a fuller picture of the advantages and disadvantages of a proposal.

(Awad et. al. 2010) 


\section{Impact of Uncertainty on Selection of Methods and Tools}

The selection of tools and methods for valuation analysis can depend on how uncertainty will be addressed in the analysis. Most tools and methods lend themselves to easily conducting sensitivity analysis. Given a plausible range of uncertain parameters, any model can be re-run to evaluate the sensitivity of the results to changes in one parameter at a time. The only barrier to conducting a sensitivity analysis is the cost and resources required to re-run the analysis with a different parameter. If many parameters are uncertain, increasing the importance of conducting a sensitivity analysis, then the choice of model should not be so complex or computationally burdensome that it prevents re-rerunning the analysis multiple times during the sensitivity analysis. Conducing a scenario analysis, where multiple parameters are changed at the same time, may require even more re-runs of the analysis.

A situation with many impactful parameters that are very uncertain also suggests a very complex and data-intensive modeling approach or tools may not be justified. With constraints on available resources, time and money might be better spent on reducing the uncertainty of key parameters rather than on employing advanced modeling techniques.

For situations in which key parameters are inherently random (but not necessarily biased), and the relationship between the output of the model and the key parameters is nonlinear, then tools should be able to accommodate time-series analysis or probabilistic methods, such as Monte Carlo simulation.

\section{Questions}

- Are there potential biases in the expected value of inputs?

If so, use sensitivity or scenario analysis to test the robustness of outcomes against a plausible range of estimates.

- Are parameters in the analysis based on processes that are inherently random?

- If so, and the system is linear, used expected value.

- If so, and the system is nonlinear (or the relationship between the inputs and outputs is not directly known, e.g., a black box model), then use techniques like time-series analysis or Monte Carlo analysis to calculate expected value.

\section{Risk Preferences and Attractiveness of Alternatives}

In many cases the uncertainty may differ among alternatives, both in the potential for bias in estimates and in variance in impacts due to inherently random processes. The variability of impacts associated with random processes is often referred to as "risk." An alternative with more gas-fired generation, for example, might be exposed to more variability in costs due to the variance in natural gas prices. For many decision makers, an alternative with the same expected value as other options but larger risk will be less attractive than alternatives with lower risk. A risk-averse decision maker might even be more interested in an alternative with a worse expected value, but lower risk. In cases with significant uncertainty or risk, the risk preferences of the decision makers should be included in the multi-criteria decision-making process in Step 5, and the tools used in the analysis should be capable of quantifying the risk associated with each alternative.

Appendix $\mathrm{G}$ includes some templates, checklists, guidance and tools that may be useful for accomplishing Step 6, including an approach for documenting how uncertainties are addressed.

\section{Stakeholder Considerations}

The effect of uncertainty on valuation assessments tends to be unrecognized or underappreciated. Often the decision regarding how to consider uncertainties is made implicitly, when analysts adopt traditional 
analysis approaches with their associated engineering and economics models. The resulting valuation may have hidden biases or even inaccuracies when the effects of uncertainty on the specific metrics of interest (Step 4) are not explicitly considered. When uncertainty is regarded as a minor technical detail, stakeholders may play limited or no direct roles in shaping Step 6, leaving it to the analysts to determine how to identify and treat the uncertainties associated with their analyses. This valuation framework requires the analysts to be deliberate in identifying uncertainties and evaluating methods to reduce the risk of an inaccurate valuation due to unknowns in data, system structures, external events, and so on. Involving stakeholders, or at least considering stakeholder perspectives, in executing and documenting Step 6 can increase the likelihood of acceptance of the valuation study results, especially when a scenariobased analysis is used.

\section{Step 6 Deliverable}

- Defined and documented approach to varying key drivers of uncertainty to produce a range of value estimates for the given alternatives.

This effort should be conducted as part of an initial study scope in conjunction with stakeholders and then revisited after an initial iteration of results from model runs and application of decision criteria. Results from initial iterations may demonstrate that certain value streams are more significant than others and may deserve further scrutiny to understand their responses - and the resulting comparison between options - to key (perhaps previously unconsidered) uncertainties.

\section{Step 7: Select Valuation Methods and Tools}

The results of the previously described activities include

- Identifying the purpose of the valuation study

- Specifying the scope of the valuation study in terms of geographic area, power sector systems/equipment, and time frame of interest

- Determining what information about system performance is required to comparatively evaluate options

- Prioritizing the performance or impact of metrics according to their importance in making a valuation assessment

- Determining how to address uncertainties inherent in the valuation process

Step 7 further develops the information flows of the valuation process and selects the methods and tools that will be employed. The potential tools (e.g., models) are selected by matching the scope and objectives of the valuation study - as expressed in the characterization of the study question and purpose (Steps 2 and 3) - with the characteristics and outputs of candidate tools or models. The methods (the strategies for simulating the system) in large part come from the equations and processes that derive often complex or customized impact metrics from the fundamental metrics that are provided by traditional power system models and analyses (Step 4).

This section describes the inputs to the method and tool selection step, specific analyses and choices to be made, and the results or deliverables that are required to complete the valuation process.

\section{How Other Process Steps Inform Step 7}

\section{Step 1: Define the Valuation Purpose}

This step has identified the key question(s) of the valuation study. In doing so, it has defined the scope of the study in terms of power system components/subsystems, geographic area, and time frame of interest. It may be helpful to express some of the scope and information requirements in tabular format. The activity of Step 1 begins to characterize the underlying valuation question or issue in order to determine the types of analyses required (e.g., production cost, generation expansion, transient stability, revenue 
requirements, reliability analyses); the elements of the power system to be considered (e.g., bulk power, distribution systems, end user); geographic limits (e.g., RTO region, utility service area, state boundary, specific substation or feeder).

\section{Step 2: Identify Alternatives to be Considered}

This step has further specified the scope of the study by defining the choices. In performing a valuation study, one focuses on the differences between alternatives, not on the overall performance of the power system. For example, if a substation's loads are projected to exceed its transformers' nameplate ratings, the alternatives might be the following:

- Installing additional transformers in the substation (or replacing the existing transformers with larger ones)

- Installing a large battery energy storage system in the substation (on the low-voltage side of the bus) or on a feeder

- Installing a specific mix (or mixes) of DER (renewable energy, fossil-fueled generators, battery energy storage, demand response systems, and/or thermal energy storage) in customer facilities served by the substation and managing/dispatching them as a microgrid

For such a study, one would look at the differences in lifecycle costs and expected reliability impacts of the alternatives, including the allocation of costs (between utility and customers served by the substation) in terms of revenue requirements and projected electric bills. It is likely not necessary to use production cost or generation expansion models to estimate overall bulk power costs and emissions.

\section{Step 3: Stakeholder Considerations}

Stakeholders generally will not be involved in directly selecting valuation methods and tools, except in cases where there may be formalized requirements regarding what kinds of analyses either are required or are not sanctioned by key grid modernization decision makers. Valuation analysts should consult with key grid modernization decision makers to ensure the analytical methods pass muster and convey to all involved stakeholders what methods will be used and why - eliciting feedback and offering to address questions. However, stakeholder considerations are very significant in determining which alternatives will be considered and in selecting and prioritizing the impact and performance metrics that will be required to do a valuation and choose among alternatives.

\section{Step 4: Identify Key Impact Metrics for Valuation}

This step has two major inputs to the selection of methods and tools:

- Basic metrics are identified. In Step 7, these will be compared with model outputs to identify which models or other tools are candidates to provide them. The scope and outputs of an analysis tool or model can also be summarized in a tabular format. A model characterization table (MCT) listing the characteristics, capabilities, and outputs of a model can easily be compared with the elements of the question characterization (from Step 1) summarizing the scope of and information (metrics) required for the valuation.

- The derivation of composite or custom metrics from the basic metrics provides equations and other methods to calculate the performance metrics, upon which valuation decisions will be based, from the basic metrics resulting from model simulation outputs.

- Analysis methods and tools are not limited to power system models: the analyst team must decide how to derive the key metrics - often very complex - from basic metrics (calculated by models) and other information sources. Regional economic impacts of adopting an alternative- such as ability to attract industry or create more jobs - often require economic models and much nonpower system-related data and assumptions. 
A major result of Step 7 will be specifying and documenting the information flows and analyses to be used to obtain the performance metrics. Therefore, the calculation of the complex/custom impact metrics from the basic (i.e., model outputs) metrics initially proposed in Step 4 will be further developed and expanded in Step 7.

Since multiple tools and methods are candidates to quantify each impact, decision maker priorities are an important first step in deciding how to allocate limited resources to constructing an analysis. In an abstract sense, the important considerations in making these choices are multidimensional trade-offs between simplicity and precision and between transparency and complexity.

Step 5: Determine Multi-Criteria Integration Approach

Step 5 explicitly deals with the trade-offs among impact metrics (the relationship, e.g., between operating cost and reliability and environmental impact.) This does not mean there has to be a quantitative relationship among impacts. For example, in choosing between two alternatives with different cost and reliability impacts, one cannot objectively equate the values of the two (e.g., $\mathrm{X}$ change in LOLP is worth $\mathrm{Y}$ increase in electricity costs $\$ / \mathrm{MWh}$ ). It is often sufficient to present clearly the results of the alternatives in terms of impact metrics (e.g., cost, reliability, emissions, land use) as a basis for discussion and debate among stakeholders. The multi-criteria integration may also include constraints as well as trade-offs among impact metrics. Such constraints and requirements may also be characterized as hard or soft. e.g., a normal rating loading constraint can be soft but an emergency rating loading constraint is hard.

The manner in which multiple criteria are viewed is influenced by the stakeholders (Step 3) and the metric prioritization (Step 5).

As part of the specification of methods, Step 7 will fully document the multi-criteria integration methodology.

Step 6: Determine Approach to Handle Uncertainty

Step 6 proposes how uncertainties will be handled. When a specific mathematical method (e.g., Monte Carlo analysis, sensitivity studies) is recommended, it may be compared with candidate models' capabilities to help select the models or other tools best for the valuation study.

The methods recommended in Step 6 selected will be finalized and fully documented as part of Step 7 .

\section{Step 7 Scope}

Previously described steps define the question and the geographic and functional scope of the valuation analysis. In so doing, they suggest certain types of models/tools (e.g., production cost, load forecast, reliability calculation, revenue requirements). Also, the impact and performance metrics upon which to base a decision are identified and prioritized; the information flows and the equations and/or analyses needed to derive the key decision metrics from basic model outputs and system data are documented; and trade-off analyses and the presentation framework needed to integrate multiple performance metrics and decision criteria are addressed.

In Step 7, the information will be further refined to compare the requirements of the analysis with the capabilities and characteristics of available tools and to continue to document the equations, information flows, and methods needed to produce decision metrics from model outputs and system data.

A way to facilitate matching study requirements with methods and tools is to create a question characterization table (QCT) to summarize the study's scope, alternatives, time frame of interest, 
systems/sectors involved, and metrics ${ }^{8}$ or other information required to support a decision. A similarly formatted MCT could summarize the scope, capabilities, and outputs of candidate methods and tools for calculating or estimating needed information. While it is not necessary to construct a QCT or MCT, doing so may help clarify what analysis methods should be considered and help match potential tools or models with what metrics must be calculated. Such an MCT could include the following:

- Model/tool type and purpose

- Sector addressed (e.g., grid, other energy systems, regional economy, environmental impacts)

- Subsystem/sector/equipment included in the model

- Geographic boundaries

- Typical time frame for study

- Time resolution for simulations

- Inputs and assumptions

- Model outputs

- How uncertainty is handled

- Model ownership and licensing requirements

Appendix $\mathrm{H}$ gives some examples of how models or tools could be characterized (in an MCT), denoting model type, scope, purpose, inputs and outputs, and other characteristics. It is meant to be a reference to assist the study team in constructing an MCT using information compatible-for comparison/matching purposes — with the information required for the study, as specified in Step 1.

There is no one correct selection of tools and methods for a valuation study. Indeed, the process of developing a valuation methodology is more of an art than a science and requires specialized expertise, as options range from highly detailed, large-scale power simulation models to simplified spreadsheet analysis based on engineering judgment and simplifying assumptions. In Step 7, the study team, with stakeholder input, must decide on the appropriate models, tools, analysis methods, decision criteria, and so on. Constraints on time, budget, available data, and personnel resources are major considerations in balancing rigor with speed budget feasibility.

The final definition of methodology and selection of tools must consider the following:

- There is a large variety of modeling tools available for the evaluation team to use. The level of details needed, data available, computing power required, team familiarity with the model, ease of use, acceptability in the wider community, and transparency of results are all factors that come into play when deciding on the model or models to be used.

- Different models will be used for different components of the valuation. The information flows and methodology must include specifying how to transfer data between models while ensuring consistency.

\section{Considering Scope and Granularity in Time, Geography, and Power System Complexity}

Key decisions must be made regarding both scope and granularity. "Scope" refers to the extent of the analysis; for time, this would be the period of time under study, from years to decades. "Geography" refers to whether the study area will be a portion of an electric system (single line, feeder, substation), the utility's entire system, or even beyond to neighboring regions or farther. "Power system scope" may mean whether to evaluate just the generation and transmission system, the distribution system, or both. Scope

\footnotetext{
${ }^{8}$ The Metrics Catalog (developed by Pacific Northwest National Laboratory) provides a comprehensive and standardized list of power system-related metrics; by using this as the basis to catalog the information needed (in the QCT) and compare with the information candidate models can provide (in the MCT), the requirements versus tool capabilities have a common lexicon for comparison.
} 
can be extended to areas including upstream fuel and water supplies, and/or downstream waste streams. Depending on the alternatives selected in Step 2, it may even include broader alternatives such as alternate resources available to provide the end-use services, such as natural gas, solar, or increased energy efficiency.

"Granularity" refers to the level of detail within the project analysis. For example, temporal granularity may be in steps of minutes, hours, weeks, months, years or longer, depending on the problem being analyzed. For example, some long-term models may only evaluate results for every 5 years, whereas more recent studies of wind and solar integration have reduced the time resolution available from hours to minutes. Geographic granularity includes whether modeling is done on a regional, sub-regional, utility, substation, feeder, or line segment basis. System granularity can expand from simply electricity to multiple voltages, and even to multi-phase versus single phase analysis. Extended system scope extends the question on fuel type to type of fuel contracts, emission types, and so on.

The purpose of the valuation will greatly influence the level of scope and granularity. As scope and granularity are increased, the difficulty in modeling, in terms of both labor and computational resources, increases greatly. It is therefore important to carefully consider the purpose for the valuation and what level of accuracy is needed, particularly given the uncertainty surrounding the inputs, while selecting the extent of scope and granularity.

Other factors to consider are that the geographic scope may need to be broader than the jurisdictional boundaries identified in Steps 2 and 3, depending on the impacts; and the adaptability of the system may propagate impacts well beyond the life of the initial decision. For horizons much beyond the present (roughly 5 or more years), serious consideration must be given to the methods used to determine future system states. Particularly long time-horizons can constrain the range of available tools for measuring certain types of impacts. Long horizons also introduce considerations of realism and cascading uncertainties. However, economic discounting of future impacts will lessen the effect of far future impacts on the overall results. The decision makers must find a balance between the availability of tools and resources and the required precision and accuracy of results, when deciding on the time horizon.

\section{Step 7 Deliverables}

The primary result of this step is an analysis strategy document that includes a process diagram of the modeling to be done, a list of the models to be used, the data requirements, and the outputs to be presented. (This could have similar detail to a request for proposals concerning the modeling and analysis.) This listing and documentation of the chosen tools, models, and other methods for determining the impacts to be considered in the analysis will take the form of a comprehensive information flow specifying the methods and tools to be used to perform the valuation analysis. Components of this include

- Methods and equations to calculate the metrics upon which the decision will be based (often complex and customized for the specific valuation study) from basic metrics (i.e., model outputs) and other data.

- Data flows among models (i.e., how some models' outputs become other models' inputs)

- An MCT of selected models (optional)

- Documented multi-criteria integration methodology (how the tools and methodologies selected will address the integration approach selected in Step 5)

- A timeline of the analysis and modeling tasks to assist in project management of the study

- The assumptions and input data required to support the above items (this is input to Step 8). 


\section{Step 8: Develop Assumptions and Input Data}

The choice of assumptions to characterize the state of the world to be analyzed will have substantial impacts on the quantitative results of the modeled alternatives. The assumptions made during a valuation process can range from structural-e.g., modeling of resource characteristics in an expansion planning/IRP process, to behavioral-e.g., whether a resource is supposed to be a price-taker or pricemaker. The impact of each assumption on the output of a valuation process is likely to be different and is sometimes known a priori. In other instances, the impact can be assessed only after the valuation process has been completed.

Transparency on these selections of assumptions and input data sets are crucial for the eventual acceptance of the valuation; stakeholders will want to be able to examine the basis behind them and potentially proffer alternate values. A few general guidelines are provided in this section; and more detailed, domain-specific guidance is documented at the method level of detail in the Valuation Methods and Tools Catalog.

- The use of common, regularly maintained data sets can streamline the selection and justification of baseline assumptions for the modeled system. A variety of these exist for the characterization of the bulk power system in long-term planning-type models. One example is the inputs to and outputs from the EIA's National Energy Modeling System (NEMS) (EIA 2009). The outputs (such as scenarios of future electricity demand and fuel prices) from the core product generated using NEMS, the EIA's AEO, are often used as inputs to many other models. Common transmission system topologies and databases of inputs to operational modeling tools (e.g., production cost models), as well as some common future planning scenarios in certain regions of the country, are available, although some have restrictions on use because of the sensitive security or commercial information included.

- Understand the capabilities and limitations of each model to be considered for the analysis. Each model has a primary purpose for which it was built. Ensure that the model represents explicitly or implicitly the physical or market mechanism that you deem important for the analysis. Often, analysts find themselves in situations in which models may not address a certain feature of a technology, so proxy values from somebody else's analysis may be used. Carefully document the reason for resorting to proxy values and justify the validity and/or level of certainty of a proxy value.

- Many commercial modeling tools include regularly updated baseline assumptions. However, they may often need to be validated and refined for region-specific analyses; and users of those tools should be aware of the provenance of the information (often, many assumptions are derived from other existing public datasets).

- Consider soliciting input from stakeholders on modeling assumptions. Many may have access to more detailed data sets at a local or sectoral level, and the process of engaging stakeholders can serve a dual purpose of improving modeling credibility and generating consensus and buy-in. This is a good point in the process of valuation to revisit whether the right stakeholders have been considered and are at the table for the analysis.

- Ensure internal consistency among all assumptions. Most often, more than a single assumption is made during the valuation process. For instance, using future natural gas prices from one source and using load growth assumption from another source may lead to internal inconsistencies. The analyst is advised to use input data from a consistent set of projections. For instance, using the results from the EIA/NEMS Reference Case for representations of future fuel price, growth, and technology choices would provide a consistent description of a future state of energy and economic systems for the United States. 
- In all cases, assumptions should be made as transparent as possible. Typically, transparency is achieved by including detailed appendices documenting assumptions and/or references to readily verifiable sources. More transparency is better - and the values of, and justification for, key assumptions (particularly those addressed in Step 9) should be included prominently in any presentation or discussion of modeling results. However, care must be taken to protect certain CEII or confidential data sources.

- To the extent possible, the analyst should identify and document the relative importance of each assumption using qualitative (or quantitative) metrics, such (1) high, (2) medium, or (3) low. The choice of assumptions and the associated input variables add risk and uncertainty to the outcome of a valuation process; hence, efforts should be made to assess sensitivity, preferably using quantitative metrics to assign the risks associated with the choice of assumptions

- Review the set of assumptions. The following are some questions that may be used to review the set of assumptions:

$\circ$ Is the assumption valid? Is the rationale behind the assumption reasonable?

○ Does the assumption duplicate or conflict with other assumptions?

- Where will the impact of an assumption be most realized in the valuation process?

- What are the key variables and metrics that reflect the impacts of assumptions?

- Which stakeholders are likely to be most impacted by the choice of assumptions/

\section{Stakeholder Considerations}

Because of their importance for the quantitative results of the modeled alternatives, analysts should ensure that the assumptions they use in conducting the valuation analyses are accurate and robust. The stakeholders involved in the valuation process to this point and subject matter experts can be invaluable in articulating and vetting these assumptions. Arguably, the stakeholders who may use the valuation analyses as input to or a basis for the grid modernization decision at issue must have full confidence in the assumptions so that the valuation results are deemed accurate, realistic, and defensible. It may be challenging to come to uniform agreement on these matters, but the analysts should work with stakeholders to do so.

\section{Step 8 Deliverable}

- Identification of all input or external data required in the information flow diagram for the study produced as a result of Step 7.

- Sources for the data identified, whether formal sources (e.g., NERC summer 2020 peak load case for load flow model) or the basis for estimates of input data. (e.g., using high, medium, and low load forecasts as a sensitivity-analysis method to address uncertainty in future loads, as decided in Step 6.)

- Assumptions inherent in the analysis. The analysis team should document all the details related to the assumptions, including any modifications to the set of assumptions made during the valuation process.

\section{Documentation of the Design Analysis Phase}

At this point, the analysts should make sure documentation of the Design Analysis phase of the valuation process is complete. Each of the steps lists deliverables that include documentation of their respective steps. The appendices associated with the steps provide checklists or templates to assist in the development of the documentation. At this point, it could be worthwhile for the decision maker and analysis team to review whether the information from Steps 6-8 necessitates an iteration back to the earlier steps 1-5 (e.g., should additional alternatives be considered, should the calculation metrics be reprioritized?). 


\subsection{Phase D: Determine the Results}

The three steps of the last phase are the calculation of key metrics, interpretation and presentation of the resulting values, and reporting of the comparison among various alternatives to guide decision making.

\section{Step 9: Assess Impacts for Each Alternative}

With the valuation study modeling framework, assumptions and input data assembled, the chain of models and analyses are executed in this step to produce quantified impacts of different metrics across the range of alternatives defined in Step 2, including the baseline case. This step can entail significant effort to run the models using the input assumptions and values, ensure that models run appropriately, and report preliminary and intermediate results to decision makers and possibly stakeholders. (In some cases, intermediate calculations include proprietary data that cannot be shared directly with some stakeholders except as a summary.) The models are run not only using baseline assumptions but also reflecting the uncertainty of those inputs using the techniques decided in Step 6, for example, through some combinations of e.g., scenario analysis, sensitivity analysis and Monte Carlo simulations. (Some of these techniques may already be incorporated in certain models.) As results start to appear, there will possibly be a need to reevaluate the models or input assumptions to improve the results relative to the original purpose and range of alternatives.

The analysis may require separate models to analyze different impacts, or the results of one model may have to be ported to another model for further analysis. This calls for intermediate data retention and documentation of the porting to ensure future validation. Some criteria may be more qualitative and require additional conversion, and uncertainties must be incorporated as described in Step 7. If other tools are used besides models (e.g., surveys, interviews) the impacts could be in multiple forms.

The impacts should be quantified over the full time-horizon for which the actions and alternatives are expected to produce differences with the baseline case. Far-distant future calculations may use simplified techniques, since their results will have fewer impacts on the total result and have greater uncertainty in their assumptions. When the policy involves an investment in a particular project, the life of the project is a good candidate for the evaluation period. An incentive program to accelerate adoption of a new technology, however, may have impacts that last much longer than the life of the incentive. In that case, a longer analysis period may be warranted. At this point, if the time horizon of the simulated alternatives does not match the chosen tool set, Step 7 should be revisited.

This step can be very labor-intensive and requires skill and expertise on the part of the analysts to evaluate model runs for their validity. Often, a significant amount of time is required to interpret complex model results and fully understand their meaning. The analyst often iterates between this Step and Step 8 by revising assumptions and input data to achieve the desired fidelity in the results.

\section{Stakeholder Considerations}

Impacts for each alternative will be assessed by valuation analysts, not by stakeholders. However, the key grid modernization decision makers and other stakeholders should be kept informed about progress in the analyses and about interim and final results. Doing so helps to keep the lines of communication open and build trust. Convening at least one session in which analysts share interim findings with involved stakeholders, and address questions and issues of concern, can be informative for those stakeholders and for analysts (e.g., perhaps these sessions will identify problems the analysts can address before results are finalized and the deliverable produced). 


\section{Step 9 Deliverable}

An assembled set of impacts for each distinct alternative. This set is likely to exist in tabular, graphical, and spreadsheet form. But, as discussed earlier, this information should not yet be used to directly compare different alternatives until a more formal set of decision criteria and valuation is applied (Step 10).

\section{Step 10: Integrate Impacts for Each Alternative}

Once the various impacts for each alternative have been quantified in Step 9, they must be integrated or summarized using the multi-criteria approach defined in Step 5. This broader comparison requires applying the defined perspective and criteria to assemble the quantitative impacts into information that supports comparison and decision making. Some comparison calculations may be done within certain models, but often they require additional processing to compare and contrast the differences from the base case. Also, the decision maker may have a broader set of criteria than that available from the results of a single model.

Depending on the method of integration and communication, this task can be as simple as totaling financial estimates, or as complicated as soliciting priorities, ranks, and weights from a variety of stakeholders. Variations on the integration criteria may be applied to better understand the perspectives of various stakeholders or simply the robustness of the integrated value. Results after applying uncertainty factors can also better inform the fully integrated results for each alternative.

\section{Stakeholder Considerations}

It is important to structure the valuation deliverable so that it is useful to and usable by stakeholders, especially stakeholders who may use valuation results in grid modernization decision making. Thus, these stakeholders should be consulted about necessary levels of detail needed and format. This consultation can occur during earlier steps (even Steps 2, 3, and 4) and informally tested during presentations of interim results in Step 9.

\section{Step 10 Deliverable}

Summary and comparison of aggregate (if applicable) and itemized value streams for the alternatives under consideration.

\section{Step 11: Compare Values, Document Analysis, and Report Findings}

A valuation study assembles relevant information, but ultimately it is the choices of decision makers outside of the valuation effort that transform that information into action. Given this, the final step of the framework is to document and discuss the valuation effort in a way that supports outside decisions.

Borrowing from the European Union's guide to CBA, a quality valuation report should be

- Self-contained: All information necessary to interpret the results is provided in the document and its appendices.

- Transparent: A complete set of data and sources of evidence should be easily available to the extent possible, without disclosing CEII or confidential data.

- Verifiable: Assumptions and methods are made available sufficiently to be replicated.

- Credible: Based on well-documented and accepted theoretical approaches and practices or, where there are deviations, they are clearly documented and justified. 
These are relatively high-level principles. To help translate them into action, we provide a list of questions that practitioners could ask themselves while assembling a report. Most of these were previously mentioned in the framework; they are consolidated here for convenience. They are also categorized by the step whose content they reflect most closely; but the grouping should be taken only as a convenient method of organizing them, not as some fundamental guidance as to how a valuation report should be organized.

Table 7. Questions to be answered in final documentation

\begin{tabular}{|c|c|}
\hline Step & Questions \\
\hline 1 & $\begin{array}{l}\text { - Are the social, institutional, and economic contexts clearly described? } \\
\text { - Are the funding source(s) and motivating entity for the valuation documented? }\end{array}$ \\
\hline 2 & $\begin{array}{l}\text { - Is the reference alternative clearly documented, and sufficiently justified as a credible option } \\
\text { that would be chosen in the absence of this project (i.e., it represents the business-as-usual } \\
\text { alternative)? } \\
\text { Does the title of the valuation study match its purpose, reference alternative, and additional } \\
\text { alternatives? } \\
\text { Are the specific details of the various alternatives (e.g., technical details of a project, language } \\
\text { of a policy) documented in a manner that allows the analysis to be replicated? }\end{array}$ \\
\hline 3 & $\begin{array}{l}\text { - Were the participants (such as stakeholders), their roles in the valuation effort, and their level of } \\
\text { involvement documented? } \\
\text { - Was the method to identify participants documented? } \\
\text { - Was the method of interaction between participants and the analysis team documented? }\end{array}$ \\
\hline 4 & $\begin{array}{l}\text { Have project effects been identified? Have sufficient effects been quantified for credibility? Is } \\
\text { the omission of potentially meaningful effects justified? } \\
\text { - Is the perspective of the valuation, and consequentially the selection of metrics, described } \\
\text { sufficiently for another group to replicate the effort? Are the choices justified? } \\
\text { Have the effects and metrics been communicated clearly and succinctly (e.g., in an easily } \\
\text { readable table)? }\end{array}$ \\
\hline 5 & $\begin{array}{l}\text { - Are the metrics that are being aggregated clearly stated? Are those metrics that are not being } \\
\text { aggregated also clearly stated? } \\
\text { If disparate metrics are aggregated together, is the methodology for doing so documented in a } \\
\text { manner that allows the calculation to be replicated? }\end{array}$ \\
\hline 6 & $\begin{array}{l}\text { Was uncertainty sufficiently explored, with results communicating the full range of possible } \\
\text { values as best as can be estimated? Are any known shortcomings in uncertainty modeling } \\
\text { documented? }\end{array}$ \\
\hline 7 & $\begin{array}{l}\text { - Are there any deviations from more widely accepted methodologies? If so, are they properly } \\
\text { justified? } \\
\text { Has the methodology been transparently documented in a manner that allows the analysis to be } \\
\text { replicated? }\end{array}$ \\
\hline 8 & $\begin{array}{l}\text { Does the analysis depend on any significant unproven assumptions (e.g., the performance of a } \\
\text { technology not yet tested at scale)? } \\
\text { - Have the input assumptions and data been documented in a manner that allows the analysis to } \\
\text { be replicated? }\end{array}$ \\
\hline 9 & - Were simplifications, caveats, and known biases in data or modeling documented? \\
\hline 10 & $\begin{array}{l}\text { - Are the values of each metric presented (i.e., if disparate metrics are aggregated together, are the } \\
\text { values of the metrics before aggregation documented)? }\end{array}$ \\
\hline General & $\begin{array}{l}\text { - Are all presentations of data in figures or tables sufficiently explained to allow a reader not } \\
\text { involved in the process to accurately determine their meaning (e.g., stating the dollar year of any } \\
\text { present-value calculations)? }\end{array}$ \\
\hline
\end{tabular}


The questions are intended to guide documentation of the analysis itself. Depending on the purpose and broader context of the valuation effort, the final report can also contain a discussion that compares the alternatives. At a minimum, a valuation report can simply present the quantification of each metric for each alternative, with any supporting text being only what is strictly necessary to interpret the results. Often, however, it is useful to provide at least some discussion of the various alternatives, to highlight the most salient points of the results and the trade-offs among alternatives. Finally, in some cases, a valuation may include an explicit recommendation or ranking, which should be discussed and documented. To be clear, a valuation effort does not need to make a recommendation to be complete; it only needs to quantify the selected impacts. However, it may be practical to leverage the knowledge of the valuation team to make a recommendation, so including a recommendation or ranking is acceptable if the entity that studied the commission requested it.

As with other steps, the assessment, integration, and comparison may result in the need to revisit earlier steps. The depth of iteration will likely depend on the time and funds available; the significance of missing alternatives, impacts, or modeling; and the amount of rigor required in the analysis.

\section{Stakeholder Considerations}

As with Step 10, it is essential to ensure that the comparisons across alternatives, documentation, and reporting of findings meet stakeholder needs relative to the grid modernization decision-making issue at hand. Continuing engagement throughout the valuation process can serve to refine and hone the initial ideas, and stakeholders typically should be provided opportunity to review or comment upon the final analyses and comparisons. So ideally, the stakeholder consultation in this step simply is an extension of the earlier engagement and a prelude to continuing engagement, if there is a need to revisit earlier steps.

\section{Step 11 Deliverable}

A final report of the analysis, presenting the quantification of the selected impacts for each alternative and providing all necessary documentation. If the entity that commissioned the study requested a recommendation or ranking, that should be included and discussed.

Appendix I includes a checklist summarizing the questions in Table 7 in a format that simplifies the review of Step 11. The checklist should be included in the document(s) comparing values, documenting the analysis, and reporting the findings.

\section{Documentation of the Determine and Present Results Phase}

At this point, the documentation of the Determine and Present Results phase of the valuation process should be completed. Each of the steps lists deliverables that include documentation of their respective steps. The appendices associated with the steps provide checklists or templates to assist in the development of the documentation. Step 11 provides the major documentation of the results, but internal documentation of the intermediate results and comparisons from Steps 9 and 10 will be useful for any follow-on analysis or validation of the study. 


\section{Review of Other Industries' Process Standards}

\subsection{Introduction}

With a long-term vision of developing a framework that could become codified as generally accepted valuation principles, several key questions must be answered:

1. Do standards exist, albeit in different industries, that successfully codify and standardize processes similar to the kind of valuation process proposed in GMLC 1.2.4?

2. If yes, what are some of the lessons learned that could be useful for directing future work towards developing commonly accepted methods for valuation?

The team reviewed standards, protocols, and guidelines for processes in other industries that had similarities to the valuation process as defined in this project. The objectives were 1) to glean insights into the applicability of standardizing or otherwise making more uniform a complex process with many actors, and 2) to assess whether some of those existing protocols and standards could serve, to some degree, as models and lessons learned for valuation of grid modernization alternatives. A companion report ${ }^{9}$ (also referenced as Appendix $\mathrm{K}$ in this report) provides more details on the standards review.

The review undertook a brief analysis of eight sets of principles, checklists or standards that are widely used in business and industry in the United States to set guidelines or establish thresholds for accuracy, transparency, consistency, repeatability, quality, and/or extensibility, including:

- The GAAP;

- International Organization for Standardization (ISO) 9000 series;

- ISO-14040 series;

- Aviation checklists;

- Medical checklists;

- American Society of Heating, Refrigerating and Air-Conditioning Engineers, Inc. (ASHRAE) Standard 202-2013;

- NERC bulk electric system reliability standards; and

- $\quad$ Rhode Island (RI) benefit-cost analysis framework. (Raab et al. 2017)

\subsection{Commonalities}

The standards and guidelines reviewed have several commonalities with the proposed valuation framework.

\section{Consistent Framework}

The purpose of most of the standards is to outline a framework requiring use of consistent methods, laying out minimum requirements and, in some cases, pointing to the need for data from reputable sources. The intent is to reduce if not eliminate the opportunity for organizations to produce results that further a narrow, special interest and/or to ensure that the organizations do not overlook key steps in the

\footnotetext{
${ }^{9}$ Cooke, Alan, et. al., Evaluation of Process-Based Standards, Pacific Northwest National Laboratory, Report XXXX, April 2019.
} 
process. For several standards, the intent is to identify the minimum set of required steps needed for success.

GAAP is literally concerned with ensuring that accurate financial reports are prepared for the use of stakeholders (e.g., investors) who require accurate and unbiased financial information that can be compared with the financial information of other entities.

ISO-9000 focuses on data-driven decision making and quality management practices, including consistent management tools and practices, timely and accurate records management, and a planning process that takes into account stakeholders (e.g., suppliers, contractors, customers, employees) and the full range of impacts.

ISO-14040 series, in particular ISO-14044, lays out a consistent framework for the analysis of environmental impacts. ISO-14044 addresses a real need that had been recognized historically when organizations made conflicting claims backed up by their "home-grown" analyses.

Checklists, aviation and medical, exist to lay out the minimum required steps to ensure that no critical component is overlooked.

NERC standards and ASHRAE 202-2013 achieve their success by ensuring that important and specific details are not overlooked and that all identified steps are taken. NERC standards set minimum thresholds for electric system reliability. ASHRAE 202-2013 describes the activities that are characteristic of best practices for achieving designed operational performance of buildings' structure and HVAC systems; it does not set quantitative requirements.

The RI framework includes a CBA step that lists benefits and costs to be analyzed as well as a policy framework identifying the specific areas that must be addressed at some level.

\section{Industry Stakeholder-Developed}

Issues faced by industry participants can be successfully addressed by standards developed by the industry.

NERC's reliability standards are developed by drafting teams composed of industry participants. Approval by a ballot body drawn from industry participants is a required step. The initiating step-a standard authorization request — can be submitted by any industry stakeholder.

ASHRAE 202-2013 is developed and maintained by a standards committee composed of the ASHRAE membership, and the process of updating the standard is generally started when a stakeholder submits a change proposal. All ASHRAE standards undergo extensive open public review.

ISO standards are also developed by committees composed of member representatives who are experts in the committee's subject area. Accordingly, ISO-9000 is developed by member representatives with quality management expertise. Standards must be approved by a membership ballot.

The RI framework was developed through a stakeholder process, and the working group came up with a unanimous, consensus proposal. (The utility regulator set it up to be a stakeholder process.)

GAAP standards or rules are developed by the Financial Accounting Standards Board (FASB); but the process includes significant amounts of stakeholder input, and one of the mechanisms for initiating a standard development/update process is a stakeholder request or recommendation. 


\subsection{Lessons Learned}

Following are some of the lessons that can be drawn from the guidelines and standards reviewed.

Brief and highly focused is better. This lesson comes from the checklists. Busy schedules, interruptions, and short attention spans are common in modern business. The best approach might be a short document that hits the essential items succinctly.

Organizational culture is a key. The ISO standards, GAAP, checklists, and NERC standards all recognize that culture is a potential issue; and they all have focus on leadership responsibility and/or how the team interacts. For example, the medical checklists have steps explicitly calling for team meetings to discuss what is being done and an approach to ensure that everyone is on the same page, so that staff do not, for example, simply arrive at an operating room, perform an operation, and leave. ${ }^{10}$

A focus on stakeholders (or "seams") is a key. The ISO and ASHRAE standards have elements that cause the organization to identify key stakeholders in the process and to either explicitly work with them, provide data to or collect data from the stakeholders, or at least reflect their perspectives in the process. ${ }^{11}$ The medical checklist team meetings are almost explicitly a "seams issue," requiring that team members coming from different medical professions talk and plan together.

ISO, ASHRAE, and NERC provide models for standard development. All are standards developed with significant opportunities for stakeholder input.

\subsection{Successful Outcomes}

The reviewed standards all demonstrated successes.

GAAP has tackled some very sticky issues, such as the true market value of an investment versus the book value, and the treatment of off-balance-sheet items such as special-purpose entities. Both issues had constituencies that quite literally fought back-including asking Congress for legislative relief. FASB can succeed at addressing such issues in part because the governing board is independent from the various stakeholder groups.

ISO-9000 successfully puts the focus on customer needs and quality management systems. Currently, over a million entities have been certified under ISO-9001 worldwide, 39,000 of which are in North America.

ISO-14044 is a credibility-building analysis insofar as it lays out a process for evaluating environmental impacts which, when paired with what is called Type III environmental declarations, can be used to credibly establish that the entity has subjected its product to an environmental assessment.

Aviation checklists are a key factor in aviation safety. The speed at which change propagates through aviation checklists is impressive. A book, The Checklist Manifesto by Atul Gawande, recounts the story of a crash and the lessons learned. It took a matter of months to complete the crash investigation, but about 10 months after the first crash, another was averted because the crew had a checklist.

\footnotetext{
${ }^{10}$ While it might be hard to believe, apparently it is not uncommon for this to happen.

${ }^{11}$ NERC standards also contain requirements for seams issues and stakeholder involvement, though individual standards' content was not explicitly discussed in this report. For example, TPL-001-4, Transmission System Planning Performance Requirements, includes requirements for working with neighboring systems' transmission planners and a stakeholder involvement process requirement related to "non-consequential load loss."
} 
Medical checklists have been demonstrated to be capable of helping hospitals all but completely eliminate errors caused by skipping or forgetting simple steps, such as washing hands or washing the patient's skin at the point of incision. Both medical and aviation checklists are potential culture changers insofar as they empower all participants to serve as backstops to other participants; and in both cases, they empower staff (e.g., a first officer or a nurse) to question the lead (pilot or doctor) to ensure that all important issues have been addressed.

ASHRAE's commissioning Standard 202 has been demonstrated to help the building industry deliver buildings that meet the advertised criteria and needs of the building occupants. It also responds to the needs of the utility industry and others who operate energy efficiency programs. Before commissioning became somewhat common, there were commonly failures in operations or performance because subsystems or processes had not been properly interfaced or because quality checks had not been performed. The ASHRAE model for setting and updating standards is a model that should be considered for the valuation framework. While the ultimate decision making rests with the committee that controls the standard, there are several opportunities for public comment as the standard is proposed, scoped, written, and updated. ASHRAE addresses all negative comments to the extent possible. The model is structured to get public comment from all stakeholders without creating an unwieldy structure.

NERC reliability standards are successful stakeholder-driven standards that have been shown to yield successes relative to historical results, such as decreasing transmission outage trends for the past 5 years and decreasing rates of protection system mis-operation. The standards themselves are developed by stakeholders, and stakeholder approval via a ballot body is a required step.

The RI framework is too new to identify a specific success resulting from the standard. However, the RI regulator that set up the process in its order accepting the stakeholder report stated that it was pleased that the process was able to achieve unanimous consensus on all but one issue.

\subsection{Considerations for Increasing Consistency of Grid Valuation Processes}

The standards review provided valuable insights into the potential for the valuation guidelines to lend themselves to being codified in some industry-adopted language or principles to improve the credibility, transparency, and overall acceptance and use of the valuation effort. The review strongly indicated that other very process-oriented activities do lend themselves to be formulated into standards language, and there is evidence that such standards or consistency in processes have improved the overall confidence in the quality of products.

It therefore appears feasible that the valuation guidelines would be codifiable by some industry body. The questions then are whether there is sufficient interest by the industry to continue such an effort, and how this effort would be structured and governed. The objective of the next phase of the GMLC Grid Valuation Framework Development project is to answer these questions and, if suitable, further advance the framework through assisted application by industry and discussions with potential requirementsdefining or standard-setting bodies. 


\section{Conclusions and Next Steps}

This project developed an 11-step valuation process in collaboration with an SAG. Several in-person meetings and webinars with SAG members confirmed the need for consistency in the process of determining the value of potential grid modernization actions (i.e., designing and executing a valuation study).

During the process development, the team tested the process on two test situations. These provided important insights into how decision makers approached valuation and what information was needed to choose among grid modernization alternatives.

We explored the applicability of establishing some form of standard for the process of grid valuation by reviewing the governance of complex processes in other disciplines. This review strongly indicates that industry groups in the buildings sector (heating, ventilation, and air conditioning), management, environmental, accounting, medical, and aviation fields have successfully established standards or standard-like guidelines that improve the quality of performance or services. The benefits from standards developed for other process-based disciplines suggest that - with sufficient interest by stakeholders of the electricity sector-similar efforts may be feasible and beneficial for evaluating grid modernization proposals.

Consistent with the vision for this project, the suggested next step would be applying this framework of an 11-step valuation process to ongoing valuation studies supporting grid modernization decisions. That step would refine and elaborate the guidelines and gauge the interest of the stakeholder community in continuing toward developing standards and best practice requirements to be applied to valuing grid modernization actions. 


\section{References}

Anderson, D., A. Eberle, T. Edmunds, J. Eto, S. Folga, S. Hadley, G. Heath, et al. 2017. Grid Modernization: Metrics Analysis (GMLC 1.1) Reference Document, Version 2.1, PNNL-26541, Pacific Northwest National Laboratory, Richland, WA, May. Available at https://gridmod.labworks.org/sites/ default/files/resources/GMLC1\%201_Reference_Manual_2\%201_final_2017_06_01_v4_wPNNLNo_1.p df. Accessed January 2019.

Awad, Mohamed L.; Keith E. Casey, Anna S. Geevarghese, Jeffrey C. Miller, A. Farrokh Rahimi, Anjali Y. Sheffrin, Mingxia Zhang, Eric Toolson, Glenn Drayton, Benjamin Hobbs, Frank Wolak. (2010). "Using Market Simulations for Economic Assessment of Transmission Upgrades: Application of the California ISO Approach", Restructured Electric Power Systems. 10.1002/9780470608555.ch7.

Boardman, Anthony, David Greenberg, Aidan Vining, David Weimer 2006. Cost-Benefit Analysis, 3rd Edition, Pearson. https://www.pearson.com/us/higher-education/product/Boardman-Cost-BenefitAnalysis-Concepts-and-Practice-3rd-Edition/9780131435834.html. Accessed January 2019.

Cooke, Alan et al. 2019, Evaluation of Process-Based Standards, Pacific Northwest National Laboratory, Report XXXX, April.

DoD (Department of Defense) 2006. Risk Management Guide for DOD Acquisition, Sixth Edition,, US Department of Defense. Available at file:///C:/Users/xce/Downloads/RM.U1.T3_Reading_2a.pdf. Accessed January 2019.

DOE (US Department of Energy) 2015. Wind Vision: A New Era for Wind Power in the United States, US Department of Energy, Washington DC, March. Available at http://www.energy.gov/sites/prod/files/ WindVision_Report_final.pdf. Accessed January 2019.

EIPC (Eastern Interconnection Planning Collaborative) 2011. Phase 1 Report: Formation of Stakeholder Process, Regional Plan Integration and Macroeconomic Analysis, Eastern Interconnection Planning Collaborative, December. Available at http://nebula.wsimg.com/44f0897683f915dac648f22ac80624b5? AccessKeyId=E28DFA42F06A3AC21303\&disposition=0\&alloworigin=1. Accessed January 2019.

EIA (US Energy Information Administration) 2009. The National Energy Modeling System: An Overview 2009, DOE/EIA-0581(2009), Energy Information Administration, Washington, DC. Available at http://www.eia.doe.gov/oiaf/aeo/overview. Accessed January 2019.

EIA (US Energy Information Administration) 2016. AEO 2016: Annual Energy Outlook 2016 with Projections to 2040, DOE/EIA-0383(2016) Energy Information Administration, Washington, DC, August 2016. Available at https://www.eia.gov/outlooks/archive/aeo16/pdf/0383(2016).pdf. Accessed January 2019.

Hirst, Eric, Martin Schweitzer 1989 "Uncertainty: A critical element of integrated resource planning", The Electricity Journal, Vol. 2, Issue 6, pp. 16-27, Elsevier B. V., July. Available at: https://doi.org/10.1016/1040-6190(89)90022-5

Kahrl, Fredrich, Andrew Mills, Luke Lavin, Nancy Ryan and Arne Olsen, 2016, The Future of Electricity Resource Planning, LBNL-1006269, Lawrence Berkeley National Laboratory, Berkeley, CA, September. Available at https://emp.lbl.gov/sites/all/files/lbnl-1006269.pdf. Accessed February 2019. 
Kintner-Meyer, M., J. Homer, P. Balducci, M. Weimar, I. Shavel, M. Hagerty, N. Powers, Y. Yang, R. Lueken 2016. Valuation of Electric Power System Services and Technologies, PNNL-25633, Pacific Northwest National Laboratory, Richland, WA.

MISO (Midcontinent ISO) 2017. MTEP17 MVP Triennial Review, Midcontinent Independent System Operator, Carmel, Indiana, September. Available at https://cdn.misoenergy.org/MTEP17\%20MVP\% 20Triennial\%20Review\%20Report117065.pdf. Accessed January 2019.

NARUC (National Association of Regulatory Utility Commissioners) 2016. NARUC Manual on Distributed Energy Resource Compensation. Draft. National Association of Regulatory Utility Commissioners, Washington, DC. Available at http://pubs.naruc.org/pub/88954963-0F01-F4D9-FBA3AC9346B18FB2. Accessed January 2019.

Puget Sound Energy 2017. 2017 Integrated Resource Plan, Appendix L: Electric Energy Storage. Available at https://www.pse.com/pages/energy-supply/resource-planning. Accessed January 2019.

Raab Associates, Paul Centolella \& Associates, Tabors Caramanis Rudkevich 2017, Docket 4600: Stakeholder Working Group Process - Report to the Rhode Island Public Utilities Commission, Raab Associates, Ltd. Boston, MA. Available at: http://www.ripuc.org/eventsactions/docket/4600WGReport_4-5-17.pdf. Accessed February 2019.

Trabish, Herman K., "How two value-of-solar studies add up to no clear value of solar: Is rooftop solar really worth 90\% less in Montana than in Maryland?" Utilitydive.com, May 10, 2018. Available at: https://www.utilitydive.com/news/how-two-value-of-solar-studies-add-up-to-no-clear-value-ofsolar/522892/. Accessed March 2019.

TVA (Tennessee Valley Authority) 2015a. Distributed Generation-Integrated Value (DG-IV): A Methodology to Value DG on the Grid, Tennessee Valley Authority, Knoxville, TN. Available at http://www.tva.gov/dgiv. Accessed January 2019.

TVA (Tennessee Valley Authority) 2015b. Integrated Resource Plan-2015 Final Report. Tennessee Valley Authority, Knoxville, TN. Available at https://www.tva.com/Environment/EnvironmentalStewardship/Integrated-Resource-Plan/2015-Integrated-Resource-Plan. Accessed January 2019.

Wilkerson, Jordan, Peter Larson, Galen Barbose, 2014, "Survey of Western U.S. electric utility resource plans", Energy Policy, Vol. 66 pp. 90-103, Elsevier B. V., March. Available at https://doi.org/10.1016/j.enpol.2013.11.029 


\section{Appendices}

Appendix A: Defining Valuation and Other Key Concepts......................................................... A-1

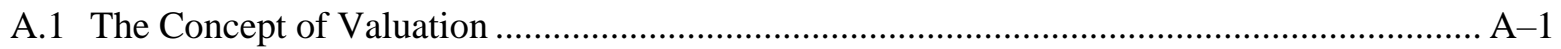

A.2 The Components and Terminology of Valuation............................................................ A -1

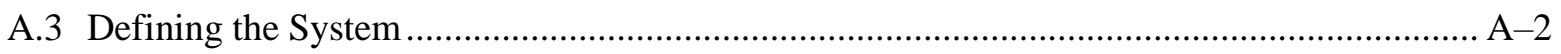

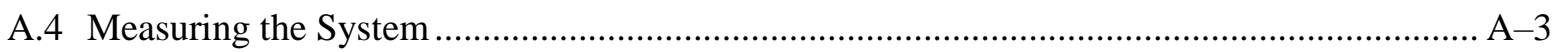

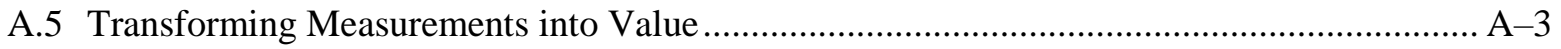

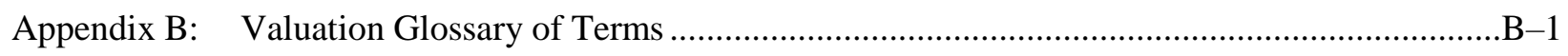

Appendix C: Tools for Step 1-Define the Valuation Question .........................................................

C.1 Checklist/Process for Scoping the Question .....................................................................

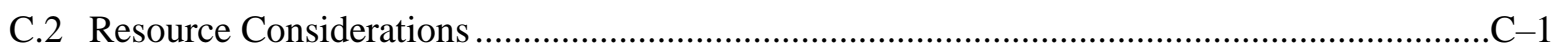

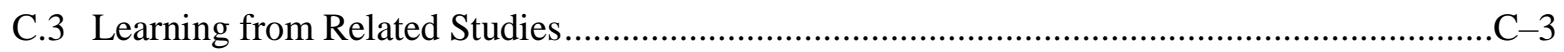

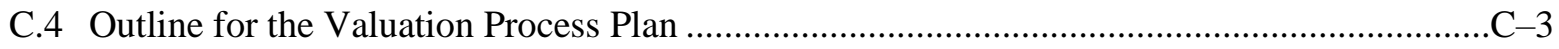

C.5 Detailed Guidance for Planning the Overall Process, Timeframe, and Milestones .................C-4 Appendix D: $\quad$ Tools for Step 2-Identifying the Range of Alternatives .......................................... D-1

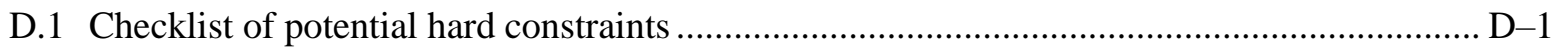

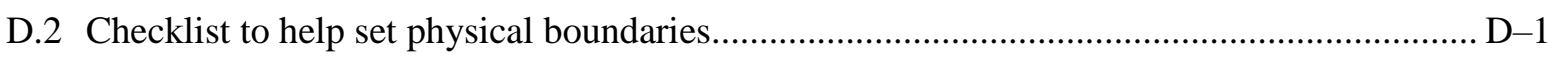

D.3 Illustrative questions for determining stakeholders.......................................................... D-2

Appendix E: Stakeholder Consideration and Engagement …...........................................................

Appendix F: Tools for Step 4-Identify Key Impact Metrics for Valuation ......................................F-1

Appendix G: $\quad$ Tools for Step 6-Determine Approach to Address Uncertainties ............................ G-1

Appendix H: $\quad$ Tools for Step 7-Select Assessment Methods and Tools........................................ H-1

H.1 Possible Format of Model Characterization Table .......................................................... H-1

H.2 Preliminary (Partial) Model Type Classification ............................................................. H-1

H.3 Sample questions for model selection guidance..............................................................

Appendix I: $\quad$ Tools for Step 11-Compare Values, Document Analysis and Report Findings............. I-1

Appendix J: Workbook for Applying the Grid Valuation Framework Process ................................... J-1

J.1 Step 1. Define the Valuation Context and Purpose ................................................................... J-3

J.2 Step 2. Identify the Range of Alternatives ........................................................................ J-4

J.3 Step 3. Plan and Initiate Stakeholder Engagement............................................................ J-5

J.4 Step 4. Identify Key Impact Metrics for Valuation............................................................ J-6

J.5 Step 5. Determine Multi-Criteria Integration Approach …................................................... J-7

J.6 Step 6. Determine Approach to Address Uncertainties........................................................ J-8

J.7 Step 7. Select Assessment Methods and Tools .........................................................................

J.8 Step 8. Develop Assumptions and Input Data................................................................... J-10

J.9 Step 9. Assess Impacts for Each Alternative.......................................................................... J-11

J.10 Step 10. Calculate Integrated Values for Each Alternative ................................................... J-12 
J.11 Step 11. Compare Values, Document Analysis, and Report Findings .................................. J-13

Appendix K: $\quad$ Evaluation of Process-Based Standards ................................................................... 



\section{Appendix A: Defining Valuation and Other Key Concepts}

To support the guidance documented in Section 3, this section lays out an abstracted definition of valuation, describing key concepts and defining key terms that are used throughout the remainder of the document. The level of abstraction used here is intended to lay the foundation of extensibility for the framework, as many of the challenges and questions that will arise from a modernizing grid are simply not yet known.

\section{A.1 The Concept of Valuation}

In a description of the challenges and needs of valuation in the electricity sector, Meyer (2017) leans on English-language dictionaries to synthesize a useful definition of valuation:

...it is a process for estimating what something is worth, relative to similar objects, entities, or services. The items that are valued through the process may have positive values (i.e., assets or benefits) or negative values (costs or liabilities)

Additionally, Kintner-Meyer et al. (2016) describe valuation more specifically in the electricity sector as:

...estimating value or worth. In the electric power sector, valuations are used when making decisions related to both the operations and planning time frames... involve countless actions, analyses, and decisions made by a wide range of stakeholders... made in order to achieve a set of goals or objectives, which often requires making tradeoffs between two or more desirable outcomes. In this way, these decisions are based on a valuation of the action to be taken that lead to a conclusion that the set of benefits of taking the action outweighs the costs.

The following concepts drawn from these two definitions are useful for informing the definition of valuation for the purposes of this guidelines document:

- Valuation is a process to generate information (estimates of value) that can support investment or other decision making with respect to future actions.

- It involves the comparison of two or more alternatives - these alternatives may be broadly construed covering changes to policies, assets, operations, and many other potential actions which can be taken by the decision maker with direct or indirect influence on the operation and planning of the power system. These alternatives are compared to each other, including typically a baseline business-as-usual case that is what is currently planned or anticipated, including "do nothing."

The following is proposed as a working definition: Valuation is a process for generating and comparing two or more alternatives for the purposes of providing information to a decision maker in support of a decision-making process.

\section{A.2 The Components and Terminology of Valuation}

Figure A-1 visually outlines some of the key high-level components of a valuation. These components, their definitions, and the function they serve in the valuation process are described herein at a high level. 


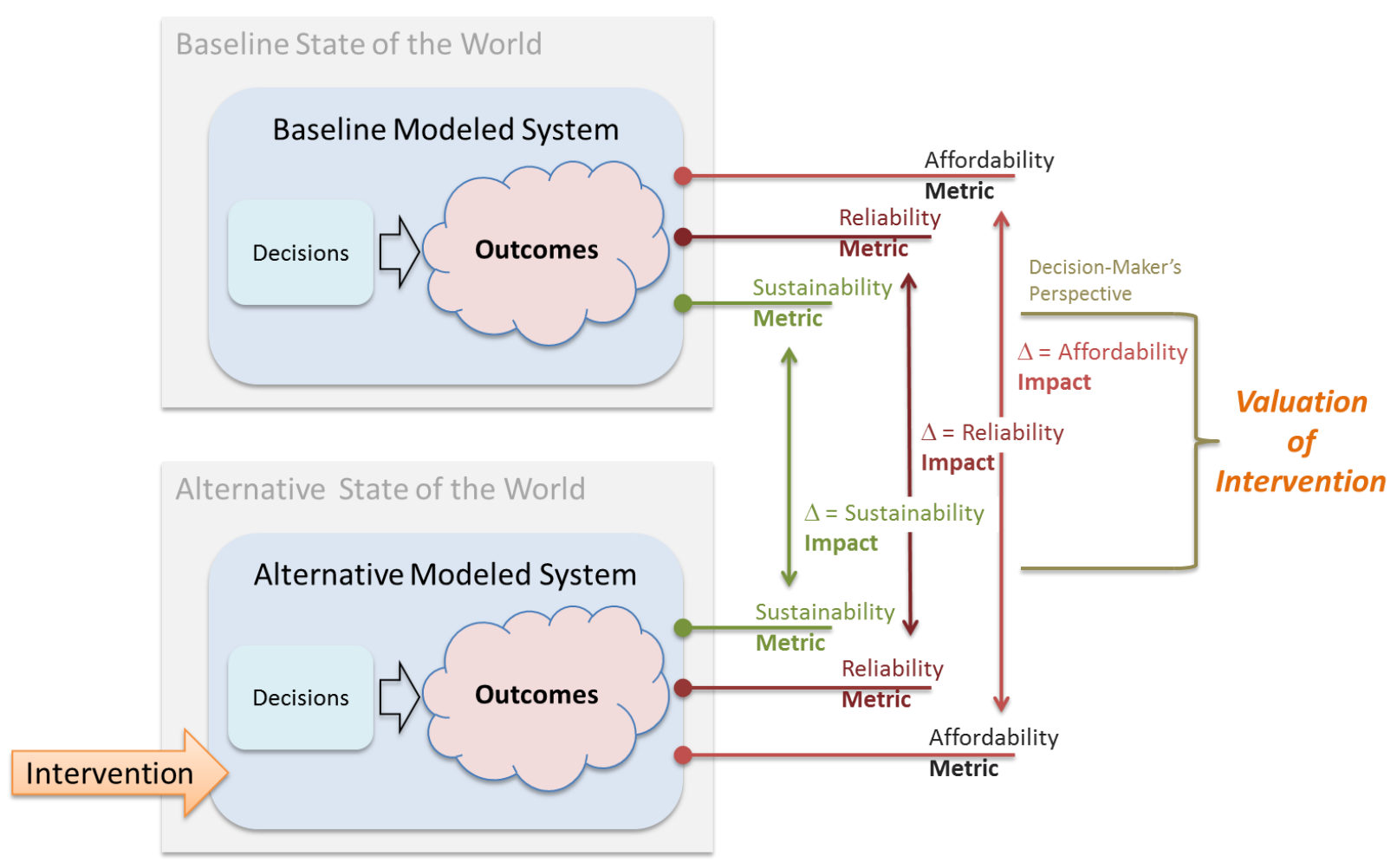

Figure A.1. Conceptual representation of the components of valuation by comparing the changes in outcomes between an alternative and a baseline.

\section{A.3 Defining the System}

Beyond the conceptual definition given above, valuation necessarily begins with the definition of the system of concern to the decision maker. In the electric sector, this system begins with the power system - which in itself is incredibly complex, spanning from individual consumers through distribution infrastructure up through transmission and generation assets at the bulk-power system level. Operational and planning decisions, prices, and physical characteristics vary along time scales from sub-second to decadal and are interconnected across political and market boundaries. The "system" can then extend to additional environmental (e.g., air, water), physical (e.g., pipeline), market (e.g., equity and commodity) and socioeconomic (e.g., employment, tax-base) contexts on which the power system acts and is in turn acted upon either directly or indirectly. The process of determining the temporal, geographic, market, and other extents of the system - that is, drawing the system boundary —is an important and clarifying step in the valuation process.

In the modeling context, the broader system is divided into the modeled system and the state of the world; the dividing line between the two is determined by which actions and properties are endogenous to the system (modeled system) and subsequently simulated and approximated, versus those that are considered for modeling purposes to be exogenous (state of the world) and are therefore fixed input assumptions that are not influenced by the alternatives within a valuation study. The precise dividing line between the modeled system and state of the world can create significant differences in outcome depending on the decision or purpose of the valuation under consideration.

A very simple example of this concept is two approaches to modeling a decision to invest in a new large baseload plant —one assuming historical prices and the other simulating power system operation using a production cost model. In the former case, the potential price-suppressing effects of new generation are "assumed away" into the state of the world (the historical price assumption), whereas a production cost 
model would resolve the market-wide changes in dispatch and marginal prices resulting from the entry and market operation of the plant in question.

Many of the real-world considerations in the choice of system size, modeling tools, and subsequent underlying assumptions are considerably more complex. Often, key assumptions concerning business principles, decision making, and human behavior are embedded into the models and tools used for valuation. These can greatly influence the results of the valuation and must be taken into account by the decision maker and stakeholders.

\section{A.4 Measuring the System}

A system property is an attribute of the system itself or its behavior. This document adopts the categorization of electric system-related properties proposed by the US Department of Energy (DOE), which bins them into six topical areas (Anderson et al. 2017):

1. Reliability-Maintaining the delivery of electric services to customers in the face of routine uncertainty in operating conditions.

2. Resilience-The ability to prepare for and adapt to changing conditions and withstand and recover rapidly from disruptions.

3. Flexibility - The ability of the grid (or a portion of it) to respond to future uncertainties that stress the system in the short term and may require the system to adapt over the long term.

4. Sustainability - The provision of electric services to customers while minimizing negative impacts on humans and the natural environment. This attribute may be more broadly defined as including three pillars: environmental, social, and economic.

5. Affordability - The ability of the system to provide electric services at a cost that is fair and affordable.

6. Security-The ability to resist external disruptions to the energy supply infrastructure caused by intentional physical or cyber attacks

System properties have subproperties defining higher granularity to its parent property. Examples include the distribution reliability subproperties of the System Average Interruption Duration Index (SAIDI) or System Average Interruption Frequency Index (SAIFI), or the sustainability subproperties of emissions of different pollutants such as $\mathrm{SO}_{2}$ or $\mathrm{CO}_{2}$. The properties and their constituent subproperties are further described in Section 3. As decisions are made within the system, the future states of system properties are collectively referred to as "outcomes."

System properties (and their subproperties) and resulting impacts are characterized for the purposes of valuation by metrics. For instance, one metric for environmental sustainability could be the emissions of a criteria pollutant at a point source over a given period. In practice, emissions of criteria pollutants would measure only a subproperty of environmental sustainability, and important decisions are made in the process of setting system boundaries with regard to which subproperties are of interest (and subsequently the metrics by which to measure them). The metrics used in this guidelines document draw heavily from work under way by a companion project in the GMLC (see Anderson et al. 2017) to catalog existing metrics used in the electricity sector and develop new metrics to support the evaluation (and valuation) of activities to modernize the grid.

\section{A.5 Transforming Measurements into Value}

As noted in the definition of valuation and in Figure A-1, for our purposes, value is a comparison of two or more future alternatives based on their respective outcomes. The "two or more" specification is important in that one set of outcomes serves as the baseline against which the possible alternatives are evaluated. As the valuation framework is designed to compare the resulting outcomes as differences 
between different interventions or actions (e.g., policies implemented, assets constructed) taken by the decision maker with respect to the power system, the baseline is typically a nonintervention business-asusual (BAU) scenario. Alternatives then are deviations from a BAU baseline. Care needs to be taken in cases where the BAU itself may be substantive, e.g. planned investments or ongoing policies.

Outcomes of two or more alternatives are compared on the basis of their difference in property or subproperty metrics; this difference in a metric is considered to be an impact, and impacts are then transformed into values based on the perspective of the decision maker. This perspective has two primary components: (1) whether a given impact is a positive relative change (a benefit), a negative (a cost), or neither and (2) the decision-making criteria that guide the ultimate choice of units by which a value is reported and interpreted and how different metrics are combined.

The impacts of each alternative are summarized in a way that informs the decision that the valuation effort is intended to support. In the simplest form, the set of impact metrics for each alternative can be summarized in a table describing the relative pros and cons of each. More sophisticated methods are available, from relatively simple graphics such as spider-diagrams to more sophisticated valuation processes. In certain cases, it is possible to monetize all impacts of interest and summarize each alternative with a single dollar value, as is done in traditional well-known approaches such as financial and social cost benefit analyses. In other circumstances - particularly when there are multiple stakeholders with differing perspectives, and monetizing impacts is considered infeasible or misleadingit may be useful to use multi-criteria weighting strategies to aggregate impacts into a single nonmonetary value or rank, with ranges to represent uncertainty or different opinions in relative weighting. Guidance related to communicating the integrated values to inform decisions is discussed in Section 3.2 under Step 5 .

Often, the robustness of a possible action is found by making variations in exogenous properties in both the BAU and alternative scenarios. For example, the influences of higher inflation, increased demand, fuel prices, and other variables may change the relative value of an alternative compared with the baseline. The "best" alternative may not be the highest in value under base assumptions but rather the alternative that appears successful under a wide range of values or minimizes the negative consequences under a plausible set of adverse assumptions.

Defining the breadth and depth of the system to be analyzed plays a central role in determining the framework of a valuation process. Valuation can encompass the narrow financial equity perspective of the decision maker, the broader perspectives of other stakeholders, or society in general. Life cycle analysis can look even more broadly, including the context of material supplies, waste streams, and interdependencies between infrastructures or sectors.

Valuation alone may not give the final decision; nonvalue factors will influence any final choice by a decision maker. These can include political issues, negotiations, timing, aesthetics, or a number of other aspects of the alternatives. While theoretically these factors could be incorporated in a valuation exercise, in reality they are more readily handled outside of the scope of valuation. In certain cases, it may be desirable to summarize impacts in a single metric that could become a de-facto decision. 


\title{
Appendix B: Valuation Glossary of Terms
}

\section{Adequacy}

The ability of a bulk power system to supply the aggregate electrical demand and energy requirements of the end-use customers at all times, taking into account scheduled and reasonably expected unscheduled outages of system components.

\begin{abstract}
Advanced Distribution Management System (ADMS)
ADMS supports the adoption levels of distributed energy resources (DER) and assists in maintaining reliability and enhance resilience across the distribution grid. ADMS add levels of communication, intelligence, and visibility into the distribution grid for the distribution utility to better understand realtime conditions across its distribution service territory. ADMS provides utilities with several specific functions, such as automated fault location, isolation, and service restoration; conservation voltage reduction; and volt/VAR optimization. Installing ADMS is not merely about better integrating DER; rather, ADMS will change how a utility operates and where a utility envisions itself and customers in the future. As customers continue to adopt technology and DER continues to grow, having the information about the grid that can be gathered from ADMS investments will help the utility meet customer demands while maintaining reliability, resilience, and flexibility. Functionally, an ADMS system integrates several utility systems, such as outage management, geographical information, AMI, and customer information systems, into one, enterprise-wide system. [NARUC 2016, p. 53]
\end{abstract}

\section{Advanced Metering Infrastructure (AMI)}

An electricity metering system that records customer's electricity consumption (and possibly other parameters) hourly or more frequently and that provides for daily or more frequent transmittal of measurements over a communication network to a central collection point. ${ }^{1}$ Advanced meters are capable of measuring consumption in 15-minute to 1-hour increments. The meters are connected to a communication network, which then transmits the consumption information to the utility's back office for billing. This stands in stark contrast to the historical mode of metering, which usually occurred once a month and included either a physical reading of the meter or collecting the information through a local radio network. With the installation of AMI, implementing electric rate designs like time of use, critical peak pricing, and real-time pricing becomes possible at lower costs than in the past. An integral part of an AMI system is a communication network. That network allows the meter to communicate with the utility and can send information like consumption and receive messages like prices or demand response signals. This two-way flow of information means that the utility can provide customers with usage, price, and cost information over the course of the month rather than only once, at the end of the month.

\section{Adverse reliability impact}

The impact of an event that results in bulk electric system instability or cascading.

\section{Affordability}

The ability of an electric system to provide electric services at a cost that does not exceed customers' willingness and ability to pay.

\section{Ancillary services}

Services that are necessary to support the transmission of capacity and energy from resources to loads while maintaining reliable operation of the transmission system in accordance with good utility practice.

\section{Area control error (ACE)}

The instantaneous difference between a balancing authority's net actual and scheduled interchange, taking into account the effects of frequency bias, correction for meter error, and automatic time error correction

\footnotetext{
${ }^{1}$ https://www.ferc.gov/industries/electric/indus-act/demand-response/dem-res-adv-metering.asp
} 
if operating in the ATEC mode. (ATEC is only applicable to balancing authorities in the Western Interconnection).

\section{Average Service Availability Index (ASAI)}

The ASAI is a reliability metric used by electric power utilities. It represents the fraction of time (often in percentage) that a customer has received power during the defined reporting period.

\section{Asset valuation methods}

Various methods such as reproduction cost and replacement cost that are used to determine the value of an asset. Other related terms include market value and earnings value.

Reproduction cost is defined as the estimated cost, usually at current prices, of duplicating an existing facility in both its current form and current function. This valuation method requires that costs be based on reproducing facilities using identical replacements; other facilities that perform the same function cannot be used. Precise reproduction costs can be difficult to calculate because some facilities may be custom-made or may be impossible to duplicate.

Replacement cost is the estimated cost, usually at current prices, of duplicating an existing facility in function only. This valuation method allows for the replacement of facilities with others that may vary considerably in form from existing facilities, while still duplicating the existing facilities' functions. The new facilities, under this method, may be redesigned to take advantage of new technology or to increase efficiency.

Market value is the value established in the market by exchanges between willing sellers and willing buyers. When a number of similar sales occur, a fairly certain market value can be determined. When a market value cannot be easily determined because of a lack of transactions, other methods such as reproduction cost or replacement cost may be used to estimate the value of property for sale.

Earnings value, also called the income or revenue method of estimating value, estimates the value of property as the present worth of future net earnings that are expected to result from the ownership of that property.

Original cost and historical cost also are used sometimes to estimate the value of an asset.

\section{Automatic generation control (AGC)}

A process designed and used to adjust a balancing authority area's demand and resources to help maintain the area control error within the bounds required by applicable NERC reliability standards.

\section{Average rates}

Average electricity or natural gas rates paid by customers over a given period of time, usually calculated either for a specific class or customers or for a specific geographical or service area.

\section{Avoided cost}

The cost that an electric utility would incur to produce or otherwise procure electric power, but does not incur because the utility purchases this power from qualifying facilities.

\section{Balancing authority}

The responsible entity that integrates resource plans ahead of time, maintains demand and resource balance within a balancing authority area, and supports interconnection frequency in real time.

\section{Balancing authority area}

The collection of generation, transmission, and loads within the metered boundaries of the balancing authority. The balancing authority maintains load-resource balance within this area.

\section{Blackout}

The disconnection of all electrical sources from all electrical loads in a specific geographic area. The cause of disconnection can be either a forced or a planned outage. 


\section{Black start capability}

The ability of a generating unit or station to go from a shutdown condition to an operating condition and start delivering electric power without assistance from the electric system.

\section{Black start resource}

A generating unit and its associated set of equipment that has the ability to be started without support from the electric system.

\section{Bulk power system}

The electrical generation resources, transmission lines, interconnections with neighboring systems, and associated equipment, generally operated at voltages of $100 \mathrm{kV}$ or higher. Radial transmission facilities serving only load with one transmission source are generally not included in this definition.

\section{Bulk power market}

A market in which large amounts of electricity at high voltages are exchanged, usually from one utility to another for the purpose of resale.

\section{Customer Average Interruption Duration Index (CAIDI)}

CAIDI is a reliability metric used by electric power utilities. It represents the average time required to restore service to customers after an outage.

\section{Customer Average Interruption Frequency Index (CAIFI)}

CAIFI is a reliability metric used by electric power utilities. It gives the average frequency of sustained interruptions for those customers experiencing sustained interruptions.

\section{Capacity charge}

The capacity charge, sometimes called demand charge, is the portion of the charge for electric service that is based on the amount of customer's peak load $(\mathrm{kW})$ within the specified billing period.

\section{Cascading}

Cascading is the uncontrolled successive loss of system elements triggered by an incident at any location. Cascading results in widespread electric service interruption that cannot be restrained from sequentially spreading beyond an area predetermined by studies.

\section{Coincidental demand or peak load}

The sum of two or more demands (or peak loads) that occur in the same time interval.

\section{Congestion}

A condition that occurs when insufficient transfer capacity is available to implement all of the preferred schedules for electricity transmission simultaneously.

\section{Conservation}

A reduction in energy consumption that corresponds with a reduction in service demand. Service demand can include buildings-sector end uses such as lighting, refrigeration, and heating; industrial processes; or vehicle transportation. Unlike energy efficiency, which is typically a technological measure, conservation is better associated with behavior. Examples of conservation include adjusting the thermostat to reduce the output of a heating unit, using occupancy sensors that turn off lights or appliances, and car-pooling.

\section{Constraints}

Constraints or system requirements are a subset of outcomes that are real-world operational requirements (or their modeling approximations) which bound the valuation process.

\section{Contingency}

The unexpected failure or outage of a system component, such as a generator, transmission line, circuit breaker, switch or other electrical element.

Single contingency - The loss of a single system element under any operating condition or anticipated mode of operation. 
Most severe single contingency-The single contingency that results in the most adverse system performance under any operating condition or anticipated mode of operation.

Multiple contingency outages-The loss of two or more system elements caused by unrelated events or by a single low-probability event occurring within a time interval too short (less than 10 minutes) to permit system adjustment in response to any of the losses.

\section{Contingency reserve}

The provision of capacity that may be deployed by the balancing authority to respond to a contingency (e.g., outage) and other contingency requirements (such as energy emergency alerts) in order to balance system generation and demand and return area control error within the specified range. Contingency reserve is typically deployed within 10 minutes following an outage. Typically, at least $50 \%$ of contingency reserve is required to be spinning reserve, which automatically responds to frequency deviations.

\section{Control area}

An area comprising an electric system or systems, bounded by interconnection metering and telemetry, capable of controlling generation to maintain its interchanges schedule with other control areas, and contributing to frequency regulation of the interconnection.

\section{Control performance standard (CPS)}

The reliability standard that sets the limits of a balancing authority's area control error over a specified time period.

\section{Curtailment}

A reduction in the scheduled capacity or energy delivery of an interchange transaction.

\section{Customer}

A metered electrical service point for which an active bill account is established at a specific location.

\section{Decision Tree}

A decision support tool that uses a tree-like graph or model of decisions, choices, options, or actions, and their possible outcomes.

\section{Demand}

The rate at which electric energy is delivered to or by a system or part of a system (at a given instant or averaged over any designated interval of time), or the rate at which energy is being used by the customer.

\section{Demand response (DR)}

Changes in electric usage by end-use customers from their normal consumption patterns in response to changes in the price of electricity over time, or to incentive payments designed to induce lower electricity use at times of high wholesale market prices or when system reliability is jeopardized. ${ }^{2}$

\section{Demand response programs}

Incentive-based programs that encourage electric power customers to temporarily reduce their demand for power at certain times in exchange for a reduction in their electricity bills. Some demand response programs allow electric power system operators to directly reduce load, while in others, customers retain control. Customer-controlled reductions in demand may involve actions such as curtailing load, operating onsite generation, or shifting electricity use to another time period. Demand response programs are one type of demand-side management, which also covers broad, less immediate programs such as the promotion of energy-efficient equipment in residential and commercial sectors.

\footnotetext{
${ }^{2}$ https://www.ferc.gov/industries/electric/indus-act/demand-response/dem-res-adv-metering.asp
} 


\section{Demand-side management (DSM)}

The term for all activities or programs undertaken by an entity (e.g., utility, customers) to influence its demand (e.g., the amount or timing of electricity they use).

\section{Direct control load management (DCLM)}

DCLM is demand-side management that is under the direct control of the system operator. DCLM may control the electric supply to individual appliances or equipment on customer premises. DCLM as defined here does not include interruptible demand.

\section{Distributed energy resources (DER)}

DER are sources of electric capacity and energy located on the distribution system or on end-user sites that feed electricity directly to buildings and end-use customers or onto the distribution grid.

\section{Distribution system}

The portion of an electric system that delivers electric energy from transformation points on the transmission system to the customer.

\section{Disturbance}

An unplanned event that produces an abnormal system condition such as high or low frequency, abnormal voltage, or oscillations in the system.

\section{Economic dispatch}

The allocation of demand to individual generating units on line to effect the most economical production of electricity.

\section{Effective load carrying capability (ELCC)}

ELCC measures the contribution of an individual generator, or group of generators, to overall resource adequacy. ELCC is a measure of the additional load that the system can supply with the particular generator of interest with no net change in reliability, as measured by LOLE or LOLP (defined later in this glossary).

\section{Electrical energy}

The generation or use of electric power by a device over a period of time, expressed in kilowatt hours $(\mathrm{kWh})$, megawatt hours (MWh), gigawatt hours (GWh), or terawatt hours (TWh).

\section{Electric power system}

A combination of generation, transmission, and distribution components.

\section{Electric rates}

The rates paid by end-use customers for electricity service. In addition to the energy charge (which is based on customers' energy consumption in $\mathrm{kWh}$ ), electric rates may also include a capacity or demand charge (based on customers' peak demand in $\mathrm{kW}$ ), and a service charge. Common types of electric rates are the following:

Flat electric rate-A flat rate charges customers per unit of consumption $(\mathrm{kWh})$, at the same rate for all units of consumption. This rate structure (in combination with a monthly customer charge) is commonly used in rates for residential electric customers. It is the most common form of residential rate design used across the country today.

Block electric rate-An increasing, inverted, or inclining block rate (IBR) structure is designed to charge customers a higher per unit rate as their usage increases over certain "blocks" within a billing cycle. For example, a three-tier IBR would identify three blocks of usage: block one could be $0 \mathrm{kWh}-150 \mathrm{kWh}$, block two $150 \mathrm{kwh}-250 \mathrm{kWh}$, and block three all usage over $250 \mathrm{kWh}$. For each block, there is a price for all electricity used within it, with the price increasing as a customer moves through the blocks over a billing period. One of the main purposes of an IBR is to send a conservation signal to customers and to incentivize energy efficiency and reduce consumption on the system. [NARUC 2016, p. 24] 
Time-variant electric rate-Time-variant rates (TVRs) are designed to recognize differences in a utility's cost of service and marginal costs at different times (e.g., hour, day, or season). Generally, a TVR design charges customers a higher price during peak hours and a lower price during off-peak hours. Unlike with flat rates, customers need to be aware of usage throughout the day and the month to respond to the price signals in a TVR design. A customer may increase savings under a TVR compared with a flat rate, if that customer uses energy in response to the time-variant price signal, such as shifting usage to lower-cost periods or conservation [NARUC 2016, p. 26]

Time-of-use (TOU) electric rate-A TOU rate is a specific kind of TVR. A TOU rate charges customers different prices according to a pre-determined schedule of peak and off-peak hours and rates. For many utilities, TOU rates have been a voluntary option for residential customers for decades, but, generally, few customers participate. Many commercial and industrial (C\&I) electric customers already receive service under TOU rate designs [NARUC 2016, p. 26]

Real time pricing $(R T P)$ rate-Under an RTP plan, the customer is charged for generation at the price set by the wholesale market (for deregulated utilities or vertically integrated utilities participating in an organized wholesale market) or at the short-run marginal generation costs (for vertically integrated utilities not participating in an organized wholesale market) by the hour. With AMI, it is possible to implement real-time pricing for residential and smaller C\&I customers. RTP is available to residential customers in the Illinois service territories for Commonwealth Edison (ComEd) and Ameren. The realtime rates for these programs are based on the day-ahead hourly wholesale price for the given utility zones. [NARUC 2016, p. 28]

Critical peak pricing (CPP) rate-A utility may implement a CPP rate during times of expected shortages or anticipated high-usage days to mimic peak time price increases. The utility will announce, usually the day before, the hours that the CPP rate will be in effect. The CPP rate reflects the higher-generation price of electricity during those CPP hours or the existence of scarcity during the event hours. Generally, the CPP rate is set significantly higher than the non-CPP rate as a means of incentivizing customers to reduce consumption. A CPP can be included with a TOU rate or paired with a demand response program. ACPP event is usually limited to certain peak hours over a year. [NARUC 2016, p. 29]

Three-part rate/demand charges-Because the utility system is built to serve peak loads, the costs of providing electricity at peak hours is higher than during non-peak hours. Part of this reflects the increased costs of having sufficient infrastructure and generation necessary to serve customers during peak demand times. To address this situation, a rate structure option is the three-part rate, which adds a demand charge to the existing fixed charge and volumetric rate. This rate recognizes three of the major contributors to a utility's costs. To the extent that each component of the rate properly reflects its associated costs, the price signal to customers should be improved over the use of flat or block rates. Such rates have been commonplace for C\&I customers. The demand charge component usually reflects the costs to provide electricity at the peak hour of the month. In an effort to identify costs associated with peak hours, a demand charge is one way for a utility to send a peak pricing signal over a certain time period (such as a month). [NARUC 2016, p. 32]

\section{Embedded cost}

The total current cost of owning, operating, and maintaining an existing electric power system.

\section{Emergency}

Any abnormal system condition that requires immediate manual or automatic action to prevent loss of firm load, equipment damage, or tripping of system elements that could adversely affect the reliability of the electric system.

\section{Energy arbitrage}

In general, storing energy when the electricity prices are low and generating when the prices are high. It typically refers to the mode of operation of pumped-storage hydropower plants in electricity markets 
when they pump during the hours with low electricity prices and generate during the hours with high electricity prices.

\section{Energy charge}

That portion of the charge for electric service based upon the electrical energy (kWh) consumed or billed.

\section{Energy efficiency}

A ratio of service provided to energy input.

\section{Energy efficiency programs}

Programs that are aimed at reducing the energy used by specific end-use devices and systems, typically without affecting the services provided.

\section{Energy intensity}

A ratio of energy consumption to another metric, typically national gross domestic product in the case of a country's energy intensity. Sector-specific intensities may refer to energy consumption per household, per unit of commercial floor space, per dollar value industrial shipment, or another metric indicative of a sector. Improvements in energy intensity include energy efficiency and conservation as well as structural factors not related to technology or behavior.

\section{Expected unserved energy (EUE)}

EUE is a reliability metric that provides information on the amount of energy shortfall that may be expected in a given time period.

\section{Federal Energy Regulatory Commission (FERC)}

FERC is the federal agency with jurisdiction over interstate electricity sales, wholesale electric rates, hydroelectric licensing, natural gas pricing, oil pipeline rates, and gas pipeline certification. FERC is an independent regulatory agency within the Department of Energy and is the successor to the Federal Power Commission.

\section{Firm power}

Power or power-producing capacity, intended to be available at all times during the period covered by a guaranteed commitment to deliver, even under adverse conditions.

\section{Fixed costs}

Costs or expenses that do not depend on the level of production output or operation and are incurred even if there is no production or operation. Fixed costs are mainly the costs of capacity, while variable costs are mainly the costs of operation.

\section{Flexibility}

The ability of an electric system to respond to future changes that may stress the system in the short term and require the system to adapt in the long term. Increased variability resulting from the growing share of variable renewable generation, such as wind and solar power, is increasing the need for flexibility in grid planning and operations.

\section{Flexibility reserve}

A new type of reserve that is being introduced in some electricity markets, mostly to compensate the variability and uncertainty of variable renewable generation (e.g., wind and solar), and to correct control area exchanges (reduce energy imbalances).

\section{Forced outage}

The condition in which the equipment is unavailable for service owing to unanticipated failure or the removal of equipment from service for emergency reasons.

\section{Frequency bias}

A value, usually given as MW/ $0.1 \mathrm{~Hz}$, associated with a balancing authority area that relates the 
difference between scheduled and actual frequency to the amount of generation required to correct the difference.

\section{Frequency control}

Also referred to as frequency regulation, frequency control includes maintaining system frequency within the specified range by continuous regulation of system generation and loads. Typically, a three-stage frequency control procedure (primary, secondary, and tertiary control) is applied.

Primary frequency control-The automatic and immediate response of turbine governors and some loads to frequency changes, which assist in stabilizing system frequency immediately following a disturbance. Primary control, also referred to as frequency response, occurs within the first few seconds following a change in system frequency.

Secondary frequency control-Balancing services deployed in the "minutes" time frame. Secondary frequency control is accomplished using the automatic generation control and the manual actions taken by the system operator to provide additional adjustments. Secondary control maintains the minute-to-minute balance throughout the day and is used to restore frequency to its scheduled value following a disturbance. Secondary control is provided by both spinning and nonspinning reserves.

Tertiary frequency control-Actions taken to provide relief for the secondary frequency control resources, so that they are available to handle current and future contingencies. Reserve deployment and reserve restoration following a disturbance are common types of tertiary control actions.

\section{Frequency regulation}

The purpose of frequency regulation, also known as frequency control, is to maintain system frequency within the specified range. Frequency regulation typically refers to both frequency response of turbine governors and to automatic generation control. It is provided by online generating units with frequencyresponsive governors and by generation and demand resources that can respond rapidly to automatic generation control requests for up and down movements to counterbalance minute-to-minute fluctuations in system load and to correct for unintended fluctuations in generator outputs.

\section{Frequency response}

The ability of a system or elements of the system to react or respond to a change in system frequency.

\section{Governor}

The electronic, digital, or mechanical device that implements primary frequency response of generating units or other system elements.

\section{Grid services}

The combination and operationalization of performance characteristics to perform a specific action, such as providing spinning reserve or load following. The commonly recognized grid services have evolved through time as new challenges have faced the grid. In a market context, performance characteristics are monetized through the procurement of select services via market products. However, not all services (e.g. inertia) currently have market products and subsequently remain unmonetized.

\section{Impacts}

Changes in outcomes as measured by metrics.

\section{Inertia}

The property of a mass that resists changes in speed.

\section{Inadvertent interchange}

The difference between the control area's net actual interchange and net scheduled interchange.

\section{Incremental cost}

The change in total costs that results when output is increased or decreased by a block or specific 
increment of units, not just by one unit. If the output is increased or decreased by just one unit (single kW or $\mathrm{kWh}$ ), the resulting costs are referred to as marginal cost.

Independent power producer (IPP)

A corporation, person, agency, authority, or other legal entity or instrumentality that owns or operates facilities for the generation of electricity for use primarily by the public, and that is not an electric utility.

Independent system operator (ISO)

An independent, federally regulated entity established to coordinate regional transmission in a nondiscriminatory manner and ensure the safety and reliability of the electric system.

Inertial response

The inertial resistance of the rotating mass of turbine generator that resists instantaneous speed changes.

Integrated resource planning (IRP)

A process of analyzing the growth and operation of utilities to ensure that energy needs are met through the optimum mix of supply-side and demand-side resources. The IRP approach is also called least-cost planning.

Interchange

Energy transfers that cross balancing authority boundaries.

Interconnected power system

A network of subsystems of generators, transmission lines, transformers, switching stations, and substations.

Interconnection

A geographic area in which the operation of bulk power system components is synchronized.

Interruptible load or demand

Demand that end-use customer makes available to its load-serving entity via contract or agreement for curtailment.

Interruption

The total loss of electric power on one or more normally energized conductors to one or more customers connected to the distribution portion of the electric system. This does not include any of the power quality issues such as sags, swells, impulses, or harmonics.

Investor-owned utility (IOU)

A privately-owned electric utility whose stock is publicly traded. It is rate regulated and authorized to achieve an allowed rate of return.

\section{Least-cost planning}

A process of analyzing the growth and operation of utilities to ensure that energy needs over a specified future period are met through the optimal (least-cost) mix of supply-side and demand-side resources, while satisfying all reliability criteria and other constraints.

\section{Load}

An end-use device or customer that receives power from the electric system.

\section{Load following}

Increase or decrease in generating unit power output to follow longer-term (hourly) changes in electricity demand.

\section{Load leveling}

Shifting the load from peak to off-peak periods, which results in a flatter load profile of system load.

\section{Load profile}

A curve depicting aggregated system load of all electricity consumers, typically over a 24-hour period. 


\section{Load-serving entity (LSE)}

Secures energy and transmission service (and related interconnected operations services) to serve the electrical demand and energy requirements of its end-use customers.

\section{Loss-of-Load-Expectation (LOLE)}

LOLE is a reliability metric derived from the loss-of-load probability (LOLP). LOLE is calculated as the sum of daily LOLP values and indicates the expected number of days during which the available generation capacity will likely not be able to fully meet the demand. LOLE is expressed in days per year. The traditional reliability target value that is used for resource adequacy is 0.1 day/year (or 1 day in 10 years).

\section{Loss-of-Load-Hours (LOLH)}

LOLH is a reliability metric derived from the loss-of-load-probability (LOLP). LOLH is calculated as the sum of hourly LOLP values and represents the number of hours during which there may be a risk of available generation not being able to fully meet the demand.

\section{Loss-of-Load-Probability (LOLP)}

LOLP is a probabilistic reliability metric commonly used in electric power system expansion planning. LOLP represents the probability that available generation capacity will not be able to fully meet the demand at a given time.

\section{Long-term transmission planning horizon}

Transmission planning period that covers years 6 through 10 or beyond when required to accommodate any known longer-term projects that may take longer than 10 years to complete.

\section{Marginal cost}

The economic concept of the change in total costs that results when output is increased or decreased by a single unit. In the electric power industry, marginal costs are the change in total costs resulting from the production of one additional kilowatt $(\mathrm{kW})$ or kilowatt-hour $(\mathrm{kWh})$ of electricity.

\section{Market clearing price}

The price at which supply equals demand for the day-ahead or hour-ahead markets.

\section{Market-based pricing}

Prices of electric power or other forms of energy determined in an open market system of supply and demand under which prices are set solely by agreement as to what buyers will pay and sellers will accept. Such prices could recover less or more than full costs, depending upon what the buyers and sellers see as their relevant opportunities and risks.

\section{Microgrid}

Microgrids are localized grids that can disconnect from the traditional grid to operate independently. Microgrids can strengthen grid resilience and help mitigate grid disturbances because of their ability to continue operating while the main electric grid is down, thereby functioning as a grid resource for faster system response and recovery.

\section{Near-term transmission planning horizon}

The transmission planning period that covers years 1 through 5 .

\section{Non-coincidental peak load}

The sum of two or more peak loads on individual systems that do not occur in the same time interval. Meaningful only when considering loads within a limited period of time, such as a day, week, month, a heating or cooling season, and usually for not more than 1 year.

\section{Nonfirm power}

Power or power-producing capacity supplied or available under a commitment having limited or no assured availability. 


\section{Nonutility power producer}

A corporation, person, agency, authority, or other legal entity or instrumentality that owns or operates facilities for electric generation and is not an electric utility. Nonutility power producers include qualifying cogenerators, qualifying small power producers, and other nonutility generators (including independent power producers). Non-utility power producers are without a designated franchised service area and do not file forms listed in the Code of Federal Regulations, Title 18, Part 141.

\section{Non-spinning reserve}

The portion of operating reserve that is not connected to the system but is capable of serving the demand within a specified time (typically within 10 minutes), or interruptible load that can be removed from the system in a specified time.

North American Electric Reliability Corporation (NERC): A nonprofit corporation formed in 2006 as the successor to the North American Electric Reliability Council established to develop and maintain mandatory reliability standards for the bulk electric system, with the fundamental goal of maintaining and improving the reliability of that system. NERC consists of regional reliability entities covering the interconnected power regions of the contiguous United States, Canada, and Mexico.

\section{Open access}

Federal Energy Regulatory Commission (FERC) Order No. 888 requires public utilities to provide nondiscriminatory transmission service over their transmission facilities to third parties to move bulk power from one point to another on a nondiscriminatory basis for a cost-based fee. Order 890 expanded open access to cover the methodology for calculating available transmission transfer capability; improvements that opened a coordinated transmission planning processes; standardization of energy and generation imbalance charges; and other reforms regarding the designation and undesignation of transmission network resources.

\section{Operating reserve}

The capability above firm system demand required to provide for regulation, load forecasting error, equipment forced and scheduled outages, and local area protection. It consists of spinning reserve and nonspinning reserve.

\section{Outage}

The period during which a generating unit, transmission line, or other facility is out of service.

\section{Outcomes}

The actual or modeled end-state of grid operations, as quantified by metrics.

\section{Peak demand}

The maximum load during a specified period of time.

\section{Performance characteristics}

The physical and operational attributes of a technology or system. Simple characteristics would include emissions rates, ramp rates, and storage capabilities. More complex characteristics might include transient responses. In a valuation context, performance characteristics must be modeled with varying levels of granularity depending on the metrics to be quantified.

\section{Planned interruption}

The loss of electric power to one or more customers that results from a planned outage.

\section{Planned outage}

The intentional disabling of a component's capability to deliver power, done at a preselected time, usually for the purposes of construction, preventive maintenance, or repair.

\section{Primary frequency response}

The immediate proportional increase or decrease in real power output provided by generating units and 
the natural real power dampening response provided by system load in response to frequency deviations. This response is in the direction that stabilizes frequency.

\section{Qualifying facility (QF)}

A cogeneration or small power production facility that meets certain ownership, operating, and efficiency criteria established by the Federal Energy Regulatory Commission pursuant to the Public Utility Regulatory Policies Act (PURPA).

\section{Ramp rate (ramping)}

The rate, expressed in megawatts per minute, at which a generator changes its output or at which the interchange schedule is attained during the ramp period.

\section{Reactive power}

The portion of electricity that establishes and sustains the electric and magnetic fields or alternatingcurrent equipment.

\section{Real power}

The portion of electricity that supplies energy to the load.

\section{Regulating margin}

The amount of spinning reserve required under nonemergency conditions by each control area to bring the area control error to zero at least once every 10 minutes and to hold the average difference over each 10-minute period to less than that control area's allowable limit for average deviation as defined by the NERC control performance criteria.

\section{Regulating reserve}

An amount of reserve responsive to automatic generation control, which is sufficient to provide normal regulating margin.

\section{Regulation service}

The process whereby one balancing authority contracts to provide corrective response to all or a portion of the area control error of another balancing authority.

\section{Reliability}

The ability of an electric power system to meet the electricity needs of end-use customers, even when unexpected equipment failures or other conditions reduce the amount of available power supply.

\section{Reserve margin (operating)}

The amount of unused available capability of an electric power system (at peak load for a utility system) as a percentage of total capability.

\section{Reserve margin (planning)}

Planning reserve margin is designed to measure the amount of generation capacity available to meet expected demand over a planning horizon. Coupled with probabilistic analysis, calculated planning reserve margins have been an industry standard used by planners for decades as a relative indication of adequacy. ${ }^{3}$ Planning reserve margin is typically expressed as the percentage by which the available generation capacity of existing and new (planned) capacity resources exceeds the net system load.

\section{Resilience}

The ability of an electric power system to resist, absorb, or withstand the impact of changes in conditions that have the potential to affect its operation, the ability to adapt in response to the change, and the ability to recover and restore system functionality rapidly.

\footnotetext{
${ }^{3}$ http://www.nerc.com/pa/RAPA/ri/Pages/PlanningReserveMargin.aspx
} 


\section{Resource planner}

The entity that develops a long-term (generally 1 year and beyond) plan for the resource adequacy of specific loads (customer demand and energy requirements) within a planning authority area.

\section{Revenue allocation}

The process of assigning to various customer classes a portion of a regulated utility's revenue requirement.

\section{Revenue requirement}

The total amount of money a regulated utility must collect from customers to pay all operating and capital costs, including a fair return on investment.

\section{System Average Interruption Duration Index (SAIDI)}

SAIDI is a reliability metric used by electric power utilities. It indicates the total duration of interruption for the average customer during a predefined period of time. It is commonly measured in minutes or hours of interruption.

\section{System Average Interruption Frequency Index (SAIFI)}

SAIFI)is a reliability metric used by electric power utilities. It indicates how often the average customer experiences a sustained interruption over a predefined period of time.

\section{Scheduled frequency}

50.0 Hertz in Europe, 60.0 Hertz in North America.

\section{Scheduled outage}

The shutdown of a generating unit, transmission line, or other facility for inspection or maintenance, in accordance with an advance schedule.

\section{Security}

The ability of an electric power system to withstand sudden disturbances such as electric short circuits, unanticipated loss of system components, or switching operations.

\section{Service territory}

The area where a utility currently provides service to retail customers, as well as specified areas adjacent to the utility's electric distribution lines or natural gas pipelines in cities and counties where the utility holds franchises.

\section{Smart inverter}

For solar photovoltaic (PV) installations, an inverter is necessary to switch electricity from direct current (DC) to alternating current (AC). The grid, including the local distribution grid, uses AC power, so before electricity generated by a solar PV installation can be exported onto the grid, it must be changed into AC. More recently, a smart inverter can now be outfitted with additional software that can accomplish additional services. For example, a smart inverter is capable of actively regulating the voltage of the solar PV output. As clouds pass over a solar PV unit, the voltage can drop on the electricity that is exported onto the grid, causing drops in voltage at that location. To raise the voltage levels, the transformer capacitor will step in and provide voltage support. Having a smart inverter address voltage drops before exporting the energy to the distribution grid is a value and service that can be provided by the customer, which can defer or avoid additional distribution upgrades. [NARUC 2016, p. 54]

\section{Spark spread}

A measurement of the difference between the price that a generator can obtain from selling one megawatt hour (MWh) of electricity and the cost of the natural gas needed to generate the MWh of electricity. Spark spread is a measure of potential profit for generating electricity on a particular day.

\section{Spinning reserve}

The portion of operating reserve consisting of the generation that is fully synchronized to the system and 
available to serve load within the specified period (typically within 10 minutes) following a contingency event.

\section{Stability}

The ability of an electric system to maintain a state of equilibrium during normal and abnormal conditions or disturbances.

\section{Supervisory control and data acquisition (SCADA)}

A system of remote control and telemetry used to monitor and control the transmission system.

\section{Supplemental reserve}

The portion of operating reserve consisting of the generation that is capable of being synchronized to the system and available to serve the load within the specified period (typically within 10 minutes) following a contingency event, or the load fully removable from the system within 10 minutes following a contingency event. It is also referred to as nonspinning reserve.

\section{Sustainability}

The ability of an electric system to provide electric services to customers with minimal impacts on natural resources, human health, or safety.

\section{Sustained interruption}

Any interruption not classified as a part of a momentary event. That is, any interruption that lasts more than 5 minutes.

\section{System}

The integrated electrical facilities, which may include generation, transmission, and distribution facilities that are controlled by one organization.

\section{System characteristics}

The current configuration of the power system. These characteristics have physical (i.e., current transmission topology and generators), regulatory (i.e., market structure), and policy (i.e., incentives) dimensions.

\section{System load}

Total aggregated demand of all electricity consumers in an electric system at a given time (e.g., instantaneous load, within a certain hour).

\section{System power value}

A forecast of the value to an electric system of the next incremental unit of power generation, usually expressed in terms of dollars per megawatt-hour for energy and dollars per kilowatt-hour for capacity.

\section{System requirements}

System requirements or constraints are a subset of outcomes which are real-world operational requirements (or their modeling approximations) that bound the valuation process.

\section{Taxonomy of grid services and technologies}

A classification scheme for grid-related technologies and services that provides a common language for the discussion of valuation.

\section{Telemetering}

The process by which measurable electrical quantities from substations and generating stations are instantaneously transmitted to the control center, and by which operating commands from the control center are transmitted to the substations and generating stations.

\section{Trade-off analysis}

Seeks to determine how the value of certain outcomes compares against the value of other outcomes, which are of different nature and measured by different metrics. 


\section{Trade-offs}

Provide information on comparative values and possible substitutions among different outcomes of different natures.

\section{Transmission}

An interconnected group of lines and associated equipment for the movement or transfer of electric energy between points of supply and points at which it is transformed for delivery to customers or is delivered to other electric systems.

\section{Transmission constraint}

A limitation on one or more transmission elements that may be reached during normal or contingency system operation.

\section{Transmission deferral}

Deferral of transmission system investments or upgrades.

\section{Unplanned interruption}

The loss of electric power to one or more customers that does not result from a planned outage.

\section{Value}

The interpretation and weighting of an outcome from a unique stakeholder perspective. Metrics for value can be quantitative or qualitative.

\section{Value of service}

A monetary measure of the value customers receive from using or consuming a specific service or product.

\section{Valuation}

The systematic process of comparing the difference in current outcomes to those of the potential introduction of a new technology, system, process, or policy.

\section{Valuation framework}

A decision tree/process by which to identify the correct tools, methods, and assumptions to model outcomes. It also provides guidance on the appropriate choice of outcomes on a technology- and stakeholder-specific basis and the level of transparency necessary for comparison and interpretation; this is done with explicit reference to the classification of technologies by their characteristics in the technology taxonomy and the configuration of the power system. Technologies or systems with similar characteristics will require similar quantification methods.

\section{Variable costs}

Costs or expenses that increase or decrease along with the increases or decreases in the level of production output or operation.

\section{Voltage collapse}

A power system at a given operating state and subject to a given disturbance undergoes voltage collapse if post-disturbance equilibrium voltages are below acceptable limits. Voltage collapse may be total (blackout) or partial and is associated with voltage instability and/or angular instability.

\section{Voltage instability}

A system state in which an increase in load, disturbance, or system change causes voltage to decay quickly or drift downward, and automatic and manual system controls are unable to halt the decay. Voltage decay may take anywhere from a few seconds to tens of minutes. Unabated voltage decay can result in angular instability or voltage collapse.

\section{Wholesale power market}

The purchase and sale of electricity from generators to resellers (who sell to retail customers) along with the ancillary services needed to maintain reliability and power quality at the transmission level. 


\section{SOURCES AND BIBLIOGRAPHY}

Energy Information Administration, Glossary of Terms. Available at http://www.eia.gov/tools/glossary/. Accessed November 16, 2016.

IEEE Standard 1366-2012, IEEE Guide for Electric Power Distribution Reliability Indices, IEEE Standards Association, New York, May 2012.

National Association of Regulatory Utility Commissioners, Distributed Energy Resources Rate Design and Compensation, November 2016.

North American Electric Reliability Corporation (NERC), Glossary of Terms Used in NERC Reliability Standards, updated September 29, 2016. Available at http://www.nerc.com/files/glossary of terms.pdf. Accessed November 8, 2016.

North American Electric Reliability Corporation, Methods to Model and Calculate Capacity Contributions of Variable Generation for Resource Adequacy Planning, March 11, 2011. Available at http://www.nerc.com/files/ivgtf1-2.pdf. Accessed November 18, 2016.

Western Electricity Coordinating Council (WECC), NERC/WECC Planning Standards and Minimum Operating Reliability Criteria - Definitions, August 9, 2002.

Northwest Public Power Association, Glossary of Electric Utility Industry Terms. Available at https://www.nwppa.org/member-resources/glossary-of-electric-utility-industry-terms/\#l. Accessed November 14, 2016.

D. Anderson et al., Grid Modernization: Metrics Analysis (GMLC 1.1), Reference Document, Pacific Northwest National Laboratory, June 2016.

Valuation of Electric Power System Services and Technologies, draft report, Pacific Northwest National Laboratory, June 2016.

Pacific Gas \& Electric, Resource-An Encyclopedia of Energy Utility Terms (Second Edition), 1992. 


\section{Appendix C: Tools for Step 1-Define the Valuation Question}

The following are the different tools developed to assist during Step 1.

\section{C.1 Checklist/Process for Scoping the Question}

\begin{tabular}{|c|c|}
\hline Considered & Components of Question \\
\hline$\bullet$ & $\begin{array}{l}\text { Who is the decision maker? } \\
\text { Who is the initiator of the valuation? } \\
\text { Who is responsible for establishing the scope and resources for analysis? } \\
\text { Who is the targeted recipient for the results and findings? }\end{array}$ \\
\hline$\bullet$ & $\begin{array}{l}\text { What is the purpose of the decision? } \\
\text { High-level investigation of policy options? } \\
\text { Real-world investment decision on technology? } \\
\text { Screening of potential alternatives? } \\
\text { Information on values for a broader audience? }\end{array}$ \\
\hline • & $\begin{array}{l}\text { What is the geographic scope of the valuation analysis? } \\
\text { What territory, region, or political entity? } \\
\text { How wide-ranging will the impacts be? } \\
\text { Which of those impacts will be examined? }\end{array}$ \\
\hline$\bullet$ & $\begin{array}{l}\text { What are the key factors and external environment that are influencing the questions? } \\
\text { Energy supply and demand } \\
\text { Relevant laws and policies } \\
\text { Important environmental issues } \\
\text { Technology breakthroughs }\end{array}$ \\
\hline$\bullet$ & $\begin{array}{l}\text { When are the impacts expected to be felt? } \\
\text { How soon? } \\
\text { For how long? }\end{array}$ \\
\hline
\end{tabular}

\section{C.2 Resource Considerations}

The valuation process pulls from a diverse set of resources that are needed to varying degrees in each of the steps. Developing a timeline, milestones, and budget requires consideration of what resources are needed and what resources are available. This can be documented through a matrix of valuation process steps and resource needs in each step, as illustrated in the example below. This example is not universally applicable to all valuation studies; but it does demonstrate the general trend that decision makers and stakeholders are more engaged early in the process, while the later steps tend to rely more on data, models, tools, and narrower grid subject-matter expertise. 
Table C.1. An example of a resource consideration matrix (more + signs indicate step requires more resources)

\begin{tabular}{|l|l|l|l|l|l|l|}
\hline \multicolumn{1}{|c|}{ Step } & $\begin{array}{c}\text { Decision- } \\
\text { makers }\end{array}$ & $\begin{array}{c}\text { Analyst } \\
\text { Team }\end{array}$ & $\begin{array}{c}\text { Stakeholder } \\
\text { Engagement }\end{array}$ & $\begin{array}{c}\text { Subject- } \\
\text { Matter } \\
\text { Expertise } \\
\text { (i.e., } \\
\text { specialists) }\end{array}$ & $\begin{array}{c}\text { Data Collection } \\
\text { and } \\
\text { Management }\end{array}$ & $\begin{array}{c}\text { Models } \\
\text { and Tools }\end{array}$ \\
\hline 1. Stakeholders & +++ & ++ & +++ & & & \\
\hline 2. Purpose & +++ & + & ++ & & & \\
\hline 3. Alternatives & ++ & +++ & +++ & ++ & & \\
\hline 4. Metrics & ++ & ++ & ++ & & & \\
\hline 5. Integration & ++ & ++ & ++ & & & \\
\hline 6. Uncertainty & + & +++ & + & ++ & & \\
\hline 7. Methods & + & +++ & + & +++ & +++ & + \\
\hline 8. Data & + & +++ & ++ & ++ & \\
\hline 9. Analysis & & +++ & & ++ & & \\
\hline 10. Values & + & +++ & + & +++ & \\
\hline 11. Report & + & +++ & + & & & \\
\hline
\end{tabular}

A similar matrix can be constructed for a particular study by answering the following questions:

- Starting with the list in the example resource consideration matrix (columns), are there any additional primary categories of resource considerations for this study? Are any of the resources not needed at all for this study?

- In which steps will key decision makers be engaged in this process? What questions will they need to provide input to?

- Are there any steps where the analyst team will be less involved than others, as they might defer more to input from decision makers, stakeholders, or grid subject-matter experts?

- When will stakeholders be engaged? What form of engagement is planned and what resources are required for that engagement? What questions will be asked of stakeholders and how will that input be managed?

- Which steps require subject matter experts to be engaged? What areas of expertise are needed to assess alternatives, impacts, assumptions, data sources, and tools and models? What questions will be asked of the experts? How will they review input assumptions, data, models, and tools? How will their expert opinions be communicated to the analyst team, stakeholders, and decisionmakers?

- How will data necessary for the analysis be collected and managed? What is the cost of collecting or procuring data? What free, publicly-available data can be used in this analysis? 
- When will models and tools be employed? How much will the tools cost to develop or procure? Are staff on the analysis team adequately trained to utilize the tools and models?

\section{C.3 Learning from Related Studies}

As related studies can be useful in many of the steps of a valuation study, an example set of key questions to answer when examining related studies, as follows, are organized in line with the overall valuation process:

\begin{tabular}{|c|c|}
\hline 1. Stakeholders & $\begin{array}{l}\text { - How were stakeholders engaged? } \\
\text { - Which stakeholders were most impacted by the alternatives? }\end{array}$ \\
\hline 2. Purpose & - What studies are available with a similar purpose to this study? \\
\hline 3. Alternatives & - What alternatives are often considered? \\
\hline 4. Metrics & $\begin{array}{l}\text { - What was the range of impacts and how were they measured? } \\
\text { - Of the full range of impacts, which were considered to be key impacts metrics for } \\
\text { analyzing in the valuation? } \\
\text { - What process was used to identify key impacts? }\end{array}$ \\
\hline 5. Integration & $\begin{array}{l}\text { - How did the study approach the integration of different impacts? Did the study attempt } \\
\text { to monetize all impacts, as in a standard cost-benefit analysis or was an alternative } \\
\text { approach used? }\end{array}$ \\
\hline 6. Uncertainty & $\begin{array}{l}\text { - What were key sources of uncertainty in the analysis? } \\
\text { - How did the study address uncertainty? }\end{array}$ \\
\hline 7. Methods & $\begin{array}{l}\text { - What assessment methods and tools were used in the analysis? } \\
\text { - What were the primary considerations for the selection of those methods or tools? }\end{array}$ \\
\hline 8. Data & $\begin{array}{l}\text { - What were the major assumptions used in the studies? } \\
\text { - What were the primary sources of data in the studies? } \\
\text { - Are any of the assumptions or data sources now out of date. If so, what impact might } \\
\text { more recent data or assumption have on the results (at least directionally)? }\end{array}$ \\
\hline 9. Analysis & $\begin{array}{l}\text { - What were the major impacts of each alternative? } \\
\text { - Are any of the quantitative impacts from each alternative useful in the current study? If } \\
\text { not directly used, can they be a useful benchmark for comparison? }\end{array}$ \\
\hline 10. Values & $\begin{array}{l}\text { - Were variations on the integration criteria applies to better understand the perspectives of } \\
\text { stakeholders? }\end{array}$ \\
\hline 11. Report & $\begin{array}{l}\text { - Given that some of the assumptions and data may now be outdated, which alternatives } \\
\text { are more attractive? } \\
\text { - Which alternatives are often not attractive? }\end{array}$ \\
\hline
\end{tabular}

\section{C.4 Outline for the Valuation Process Plan}

The Valuation Process Plan is a deliverable that summarizes the purpose, question statement, process, time frame, and milestones for the valuation study. In effect, it is similar to a Statement of Work for the project.

\section{C.4.1 Purpose}

What is the purpose of the valuation study? Is it a high-level investigation of policy options? Is the purpose to inform a real-world investment decision for a particular technology? Is it intended to screen potential alternatives? Or is it providing information on values for a broader audience? 


\section{C.4.2 External Environment}

What are some of the key factors and external environment that are influencing the questions being asked in the study? Are there changes in energy supply and demand? Are there particular laws and policies that are driving the need to compare alternative courses of action? Are there emerging environmental issues that need to be addressed? Or are there technology breakthroughs that may change the relative attractiveness of business-as-usual pathways?

\section{C.4.3 Timeframe}

Over what period will the valuation study be conducted? What are the key considerations in establishing the timeframe?

\section{C.4.4 Resources}

At a high-level, what resources are available for the study? Where are there major constraints in resources that affect the scope and level of detail of the study?

\section{C.4.5 Deliverables and Schedule}

What are the key deliverables and when are they expected to be delivered?

\section{C.5 Detailed Guidance for Planning the Overall Process, Timeframe, and Milestones}

\section{Basic outline}

Establish the time when the decision or findings are needed

Establish the resources available (funding, personnel)

Set up a chart for all major steps in the process

Establish milestones and deliverables at multiple points through the process

\section{Key questions to examined}

- What resources are needed to answer the question?

- Funding

- Personnel

- When does the valuation need to be completed?

- What major milestones are required?

- How should the valuation process be documented?

- Interim reports

- Final documentation

- Other 


\section{Appendix D: Tools for Step 2-Identifying the Range of Alternatives}

The following are the different tools developed to assist during Step 2.

\section{D.1 Checklist of potential hard constraints}

\begin{tabular}{|c|l|l|}
\hline Considered & Constraint & Type of Constraint \\
\hline$\bullet$ & Renewable Portfolio Standard & Policy \\
\hline$\bullet$ & Non-wire alternatives/efficiency first & Policy \\
\hline$\bullet$ & $\begin{array}{l}\text { Technology mandates (e.g., California's energy storage mandate } \\
\text { disallows energy storage technologies greater than 50 MW in size) }\end{array}$ & Policy \\
\hline$\bullet$ & NERC reliability standards & Regulatory \\
\hline & Budget/cost & Financial \\
\hline$\bullet$ & Environmental Protection Agency emissions, water quality & Regulatory \\
\hline
\end{tabular}

\section{D.2 Checklist to help set physical boundaries}

\begin{tabular}{|c|c|c|}
\hline Considered & Basis of determination & Comments \\
\hline$\bullet$ & $\begin{array}{l}\text { Locational attributes of } \\
\text { the service to be } \\
\text { provided; for instance, } \\
\text { distribution vs. bulk } \\
\text { system }\end{array}$ & $\begin{array}{l}\text { Generating capacity and reserves can be provided by } \\
\text { resources throughout a region. Reactive power support is } \\
\text { most efficiently provided by local resources. }\end{array}$ \\
\hline$\bullet$ & $\begin{array}{l}\text { Calculation of } \\
\text { reliability metrics; } \\
\text { NERC reliability region }\end{array}$ & $\begin{array}{l}\text { Area control error (ACE) is calculated and monitored for } \\
\text { individual BAs, but frequency response is measured on an } \\
\text { interconnection basis (BAL-003-01) and hence allows BAs to } \\
\text { meet their respective obligations by contracting with entities } \\
\text { in other BAs that have excess primary frequency responsive } \\
\text { capacity }\end{array}$ \\
\hline$\bullet$ & $\begin{array}{l}\text { Market footprint- } \\
\text { ISO/RTO regions }\end{array}$ & $\begin{array}{l}\text { Resources outside an independent system operator } \\
\text { (ISO)/RTO footprint can be viable alternatives through } \\
\text { imports of energy/reserve capacity, given availability of } \\
\text { adequate transmission capacity, and so on. }\end{array}$ \\
\hline$\bullet$ & $\begin{array}{l}\text { Market rules- } \\
\text { ISO/RTO regions }\end{array}$ & $\begin{array}{l}\text { DER participation in ISO/RTO markets is limited by location } \\
\text { within the distribution system, generally requiring } \\
\text { aggregation up to minimum capacity levels, such as at least } \\
100 \mathrm{~kW} \text { within a single sub-LAP (load aggregation point) in } \\
\text { CAISO (California Independent System Operator) }\end{array}$ \\
\hline$\bullet$ & $\begin{array}{l}\text { Locational attributes of } \\
\text { the service to be }\end{array}$ & $\begin{array}{l}\text { RA capacity may be provided by resources located in either } \\
\text { distribution or transmission systems. Similarly, reserve }\end{array}$ \\
\hline
\end{tabular}




\begin{tabular}{|c|l|l|}
\hline Considered & Basis of determination & Comments \\
\hline & $\begin{array}{l}\text { provided; for instance, } \\
\text { distribution vs. bulk } \\
\text { system }\end{array}$ & $\begin{array}{l}\text { capacity can be provided by resources throughout a region, } \\
\text { but reactive power support can only be provided by local } \\
\text { resources. }\end{array}$ \\
\hline$\bullet$ & $\begin{array}{l}\text { Calculation of } \\
\text { reliability metrics; } \\
\text { NERC reliability region }\end{array}$ & $\begin{array}{l}\text { ACE is calculated and monitored for individual BAs, but } \\
\text { frequency response is measured on an interconnection basis } \\
\text { (BAL-003-01) and hence allows BAs to meet their respective } \\
\text { obligations by contracting with entities in other BAs that have } \\
\text { excess primary frequency responsive capacity }\end{array}$ \\
\hline$\bullet$ & $\begin{array}{l}\text { Market footprint }- \\
\text { ISO/RTO regions }\end{array}$ & $\begin{array}{l}\text { Resources outside an ISO/RTO footprint can be viable } \\
\text { alternatives through imports of energy/reserve capacity, given } \\
\text { availability of adequate transmission capacity and so on. }\end{array}$ \\
\hline & $\begin{array}{l}\text { Market rules - } \\
\text { ISO/RTO regions }\end{array}$ & $\begin{array}{l}\text { DER participation in ISO/RTO markets is limited by location } \\
\text { within the distribution system, generally requiring } \\
\text { aggregation up to minimum capacity levels, such as at least } \\
\text { 100 kW within a single sub-LAP in CAISO }\end{array}$ \\
\hline
\end{tabular}

\section{D.3 Illustrative questions for determining stakeholders}

Focus is an energy-saving technology or program:

- Who is responsible for energy savings?

- Who pays utility bills?

- Who receives' the savings?

- Who controls those savings?

- Who owns the new equipment?

- Who is responsible for its maintenance and repair?

- Who is the end user?

Focus is a change in rate structures:

- Who is responsible for decisions to alter rate structures?

- Who can influence rate amounts?

- Who benefits from new rates?

- Initially, and over time?

- Direct and indirect beneficiaries?

○ What constitutes "benefits" for these parties?

- Who is harmed by new rates? How?

- Initially, and over time?

$\circ$ Directly and indirectly? Who bears the costs? 
- What constitutes harms for these parties?

$\circ$ Who determines any mitigating or compensatory actions?

$\circ$ Who is responsible - for paying, implementing, monitoring?

- Who determines the degree to which the distribution of benefits and harms is fair or appropriate?

Focus is a policy change that can change cost/benefit profiles for multiple technologies:

- Who (what organization, or portion of the organization) is responsible for making this policy change?

- Who is responsible for implementing this policy change?

○ Who pays? (initially and over time?)

$\circ$ Who receives revenues or savings?

- Who reports, monitors, evaluates?

○ Is policy dependent upon other entities' actions - if so, which entities?

- Who benefits from new policy?

- Initially and over time?

- Within sponsoring or responsible organization? (and across levels of the organization-e.g., local vs. regional vs. national)

- Entities outside of sponsoring or responsible organization?

- What constitutes benefits for these parties?

- Who is harmed by new policy? How?

- Initially and over time?

- Within sponsoring or responsible organization? (And across levels of the organization-e.g., local vs. regional vs. national)

- Entities outside of sponsoring or responsible organization? (at different locations or levels of organization-e.g., local vs. regional vs. national)

- What constitutes harm for these parties?

- Who determines the degree to which the distribution of benefits and harms is fair or appropriate? 



\section{Appendix E: Stakeholder Consideration and Engagement}

\section{Stakeholder Consideration Sufficiency Checklist}

Checklist for use in identifying stakeholders and their respective area of interest.

\begin{tabular}{|l|l|l|}
\hline & Internal Stakeholder Category & Points of Contact \\
\hline$\bullet$ & Finance & \\
\hline$\bullet$ & Engineering & \\
\hline$\bullet$ & Legal & \\
\hline$\bullet$ & Human Resources & \\
\hline$\bullet$ & Operations & \\
\hline$\bullet$ & Planning & \\
\hline$\bullet$ & Labor & \\
\hline
\end{tabular}

\begin{tabular}{|l|l|l|}
\hline & External Stakeholder Category & Points of Contact \\
\hline$\bullet$ & Electricity End-User (Rate-payers) & \\
\hline$\bullet$ & Load-Serving Entities (LSEs) & \\
\hline$\bullet$ & $\begin{array}{l}\text { Grid Infrastructure Asset-Owners (Generation, } \\
\text { Transmission, Distribution) }\end{array}$ & \\
\hline$\bullet$ & Utility Commissions & \\
\hline$\bullet$ & Independent System Operators & \\
\hline$\bullet$ & Market Operators & \\
\hline$\bullet$ & Technology OEMs and Third-Party Providers & \\
\hline$\bullet$ & $\begin{array}{l}\text { Environmental and Energy Interest Groups and } \\
\text { Regulators }\end{array}$ & \\
\hline$\bullet$ & Other Interest Groups and Regulators & \\
\hline
\end{tabular}

Impacted v. Influential in the Outcome Guidance

Who is impacted by this project? Note that this question is trying to identify all the categories of stakeholders who might be impacted by the project.

Specifically,

a. Who realizes benefits?

- Utility customers?

- Utilities

- Visitors

- Other entities connected to the same grid

- Governmental entities

- Society within the service territory

- Society as a whole 
- Others?

b. If this work were not being paid for by DOE, who would pay for it?

- Customer themselves

- Utilities assigning costs to all customers

- Taxpayers

- Others

c. Are there any other stakeholders who should be considered? 


\section{Appendix F: Tools for Step 4-Identify Key Impact Metrics for Valuation}

Checklist of potential grid metrics for consideration by system property

\begin{tabular}{|c|c|c|c|c|}
\hline Category & & & & \\
\hline \multirow[t]{3}{*}{ Reliability } & $\begin{array}{l}\text { SAIDI (System } \\
\text { Average } \\
\text { Interruption } \\
\text { Duration Index) }\end{array}$ & $\begin{array}{l}\text { SAIFI (System } \\
\text { Average } \\
\text { Interruption } \\
\text { Frequency Index) }\end{array}$ & $\begin{array}{l}\text { MAIFI } \\
\text { (Momentary } \\
\text { Average } \\
\text { Interruption } \\
\text { Frequency Index) }\end{array}$ & $\begin{array}{l}\text { Monthly CPS } \\
\text { (Control Performance } \\
\text { Standard 1) }\end{array}$ \\
\hline & $\begin{array}{l}\text { Monthly BAAL } \\
\text { (Balancing } \\
\text { Authority ACE } \\
\text { Limit) }\end{array}$ & $\begin{array}{l}\text { Daily IROL } \\
\text { (Interconnection } \\
\text { Reliability } \\
\text { Operating Limit) }\end{array}$ & $\begin{array}{l}\text { Daily SOL (System } \\
\text { Operating Limit) } \\
\text { violations }\end{array}$ & $\begin{array}{l}\text { Annual SRI (Severity } \\
\text { Risk Index) }\end{array}$ \\
\hline & $\begin{array}{l}\text { LOLE (Loss of } \\
\text { Load } \\
\text { Expectation) }\end{array}$ & $\begin{array}{l}\text { Required reserve } \\
\text { margin }\end{array}$ & & \\
\hline \multirow[t]{2}{*}{ Resilience } & $\begin{array}{l}\text { Total time to } \\
\text { recovery }\end{array}$ & $\begin{array}{l}\text { Power demand not } \\
\text { met during the } \\
\text { disruption (e.g., } \\
\text { measured in total } \\
\text { megawatt-hours) }\end{array}$ & $\begin{array}{l}\text { Number of people } \\
\text { without power } \\
\text { (e.g., in total } \\
\text { person-hours) }\end{array}$ & $\begin{array}{l}\text { Impact on critical } \\
\text { infrastructure (e.g., } \\
\text { number of emergency } \\
\text { services facilities } \\
\text { without power, } \\
\text { fueling infrastructure, } \\
\text { communications } \\
\text { infrastructure) }\end{array}$ \\
\hline & $\begin{array}{l}\text { Cost of response } \\
\text { and recovery } \\
\text { activities }\end{array}$ & $\begin{array}{l}\text { Grid revenue } \\
\text { losses }\end{array}$ & Economic impacts & \\
\hline \multirow[t]{3}{*}{ Flexibility } & $\begin{array}{l}\text { Unserved load or } \\
\text { reserves }\end{array}$ & $\begin{array}{l}\text { Poor balancing } \\
\text { control } \\
\text { performance (e.g., } \\
\text { low CPS1 scores) }\end{array}$ & $\begin{array}{l}\text { Renewable } \\
\text { curtailment }\end{array}$ & $\begin{array}{l}\text { Wholesale price } \\
\text { volatility including } \\
\text { negative prices }\end{array}$ \\
\hline & $\begin{array}{l}\text { Constrained } \\
\text { ramp rates }\end{array}$ & $\begin{array}{l}\text { Loss-of-load } \\
\text { probability }\end{array}$ & $\begin{array}{l}\text { Expected unserved } \\
\text { energy }\end{array}$ & Load forecast error \\
\hline & Headroom & Price volatility & $\begin{array}{l}\text { Insufficient } \\
\text { ramping resource } \\
\text { expectation }\end{array}$ & Load shedding \\
\hline \multirow[t]{3}{*}{ Sustainability } & $\begin{array}{l}\text { Greenhouse gas } \\
\text { emissions }\end{array}$ & $\mathrm{N}_{2} \mathrm{O}$ emissions & $\mathrm{CH}_{4}$ emissions & $\begin{array}{l}\text { Hydrofluorocarbon } \\
\text { emissions }\end{array}$ \\
\hline & $\begin{array}{l}\text { Perfluorinated } \\
\text { compound } \\
\text { emissions }\end{array}$ & $\mathrm{SF}_{6}$ emissions & $\mathrm{NF}_{3}$ emissions & $\begin{array}{l}\text { Pollutant discharges } \\
\text { to water }\end{array}$ \\
\hline & Land-use change & $\begin{array}{l}\text { Depletion of } \\
\text { natural resources }\end{array}$ & $\begin{array}{l}\text { Impact on human } \\
\text { health }\end{array}$ & $\begin{array}{l}\text { Impact on global } \\
\text { average temperature }\end{array}$ \\
\hline
\end{tabular}




\begin{tabular}{|c|c|c|c|c|}
\hline \multirow[t]{3}{*}{ Affordability } & $\begin{array}{l}\text { Levelized cost of } \\
\text { electricity }\end{array}$ & $\begin{array}{l}\text { Proportion of } \\
\text { electricity costs to } \\
\text { household income }\end{array}$ & $\begin{array}{l}\text { Proportion of } \\
\text { electricity costs to } \\
\text { gross revenue }\end{array}$ & Avoided costs \\
\hline & $\begin{array}{l}\text { Affordability gap } \\
\text { index }\end{array}$ & $\begin{array}{l}\text { Affordability } \\
\text { headcount index }\end{array}$ & Total system cost & $\begin{array}{l}\text { Impact on utility cash } \\
\text { flows (e.g., taxes and } \\
\text { depreciation) }\end{array}$ \\
\hline & $\begin{array}{l}\text { Impact on region } \\
\text { outside the scope } \\
\text { of the study (e.g., } \\
\text { regional plan) }\end{array}$ & $\begin{array}{l}\text { Economic factors } \\
\text { (e.g., GDP growth, } \\
\text { jobs) }\end{array}$ & & \\
\hline \multirow[t]{2}{*}{ Security } & $\begin{array}{l}\text { Annualized loss } \\
\text { expectancy }\end{array}$ & $\begin{array}{l}\text { Reportable } \\
\text { cybersecurity } \\
\text { incidents }\end{array}$ & $\begin{array}{l}\text { Reportable } \\
\text { physical security } \\
\text { events }\end{array}$ & $\begin{array}{l}\text { Incidents requiring } \\
\text { manual cleanup }\end{array}$ \\
\hline & Mean-time-to-fix & $\begin{array}{l}\text { Mean cost to } \\
\text { mitigate } \\
\text { vulnerabilities }\end{array}$ & & \\
\hline
\end{tabular}

Source: Adapted from Anderson et al., Grid Modernization: Metrics Analysis Reference Document, Pacific Northwest National Laboratory, June 2017. 


\section{Appendix G: Tools for Step 6-Determine Approach to Address Uncertainties}

The checklist suggests different methods of addressing uncertainty during Step 6.

Encompassing a Realistic Solution Space Checklist

\begin{tabular}{|c|l|l|}
\hline Considered & Uncertainty & \\
\hline$\bullet$ & Demand growth & Multiple scenarios, planning reserve margin \\
\hline$\bullet$ & Generator outages & $\begin{array}{l}\text { Outage rates applied to random availability or } \\
\text { derating, operating reserve margins }\end{array}$ \\
\hline$\bullet$ & $\begin{array}{l}\text { Generation and transmission } \\
\text { equipment failure }\end{array}$ & N-1 criteria \\
\hline$\bullet$ & $\begin{array}{l}\text { Fuel availability (fossil, } \\
\text { renewable) }\end{array}$ & Minimum supply, capacity penalty \\
\hline$\bullet$ & Inflation & Multiple scenarios \\
\hline$\bullet$ & Construction delays & Multiple scenarios \\
\hline$\bullet$ & Distribution outages & \\
\hline
\end{tabular}





\section{Appendix H: Tools for Step 7-Select Assessment Methods and Tools}

\section{H.1 Possible Format of Model Characterization Table}

A model characterization table should contain the following information:

- Model type

- Model name

- Typical purpose/use of this type of model

- Power sector subsystem modeled

- Geographic area

- Time frame for study/simulation

- Time step/resolution for simulations

- Model outputs

- Model inputs - generic (this will reference generic inputs for this type of model)

- Model inputs - specific (model-specific inputs)

- Characterization or optimization?

- Algorithms used (handling uncertainty, making calculations)

- Model ownership (reference and licensing information)

- Text description/comments

\section{H.2 Preliminary (Partial) Model Type Classification}

- Regional economic models

- Capacity expansion

- Production cost

- Load flow, voltage drop, transient stability

- Reliability

- Load forecast/load models

- Tariffs and pricing

- Environmental impacts

- Weather

- Power quality

\section{H.3 Sample questions for model selection guidance}

- What is the question being asked about: technology or policy?

- What value streams are to be considered in the overall valuation: e.g., avoided energy, generation capacity deferral, generation operation and maintenance, system losses, transmission system impact, distribution system impact, reserves, environmental impact, ancillary services, economic development impact, security enhancement, disaster recovery?

- What sector is the question addressing: generation, transmission, distribution, end-use?

- What other sectors need to be included in the modeling: e.g., fuel, water, air, generation, transmission, distribution, end-use, consumer behavior?

- What technical details need to be modeled: transformers, individual lines, multiple voltages, individual generators, generator types, plant emissions, storage? 
- What financial cost details need to be modeled: operating, construction, business finances, pricing, price elasticities, multiple rate classes, taxes, tax credits, other policies?

- What territory is to be modeled: national, regional, state, utility, substation, feeder, neighborhood, other?

- What time duration needs to be modeled: weeks, months, years, decades?

- What time increments need to be modeled: cycles, seconds, minutes, hours, days, weeks, months, years?

- How are time-dependent variations to be captured: time-sequenced, peak demands, dynamic flow, load duration curves, static analyses?

- How will uncertainty be captured: probabilistic modeling, multiple scenarios, sensitivities?

- How much input data is available?

- How much time is available until results are required?

- What resources are available for the analysis and modeling: funding, labor, computer power, software? 


\section{Appendix I: Tools for Step 11-Compare Values, Document Analysis and Report Findings}

\section{Report Sufficiency Considerations Checklist}

Checklist of items that should be included in the document(s) comparing values, documenting the analysis and reporting the findings.

Step 1: Is the social, institutional, and economic context clearly described?

Step 1: Are the funding source(s) and motivating entity for the valuation documented?

Step 2: Is the reference alternative clearly documented, and sufficiently justified as a credible option that would be taken in the absence of this project (i.e., it represents the business-as-usual alternative)?

Step 2: Does the title of the valuation study match its purpose, reference alternative, and additional alternatives?

Step 2: Are the specific details of the various alternatives (e.g., technical details of a project, language of a policy) documented in a manner that allows the analysis to be replicated?

Step 3: Were the participants (such as stakeholders), their roles in the valuation effort, and their level of involvement documented?

Step 3: Was the method to identify participants documented?

Step 3: Was the method of interaction between participants and the analysis team documented?

Step 4: Have project effects been identified? Have sufficient effects been quantified for credibility? Is the omission of potentially meaningful effects justified?

Step 4: Is the perspective of the valuation, and consequentially the selection of metrics, described sufficiently for another group to replicate the effort? Are the choices justified?

Step 4: Have the effects and metrics been communicated clearly and succinctly (e.g., an easily readable table)?

Step 5: Are the metrics that are being aggregated clearly stated? Are those metrics that are not being aggregated also clearly stated?

Step 5: If disparate metrics are aggregated together, is the methodology for doing so documented in a manner that allows the calculation to be replicated?

Step 6: Was uncertainty sufficiently explored, with results communicating the full range of possible values as best as can be estimated? Are any known shortcomings in uncertainty modeling documented?

Step 7: Are there any deviations from more widely-accepted methodologies? If so, are they properly justified?

Step 7: Has the methodology been transparently documented in a manner that allows the analysis to be replicated?

Step 8: Does the analysis depend on any significant unproven assumptions (e.g., the performance of a technology not yet tested at scale)?

Step 8: Have the input assumptions and data been documented in a manner that allows the analysis to be replicated? 
Step 9: Were simplifications, caveats, and known biases, in data or modeling documented?

Step 10: Are the values of each metric presented (i.e., if disparate metrics are aggregated together, are the values of the metrics prior to aggregation documented)?

General: Are all presentations of data in figures or tables sufficiently explained to allow a reader not involved in the process to accurately determine their meaning (e.g., stating the dollar year of any present-value calculations). 


\section{Appendix J: Workbook for Applying the Grid Valuation Framework Process}

\section{Overall Valuation Framework Process}

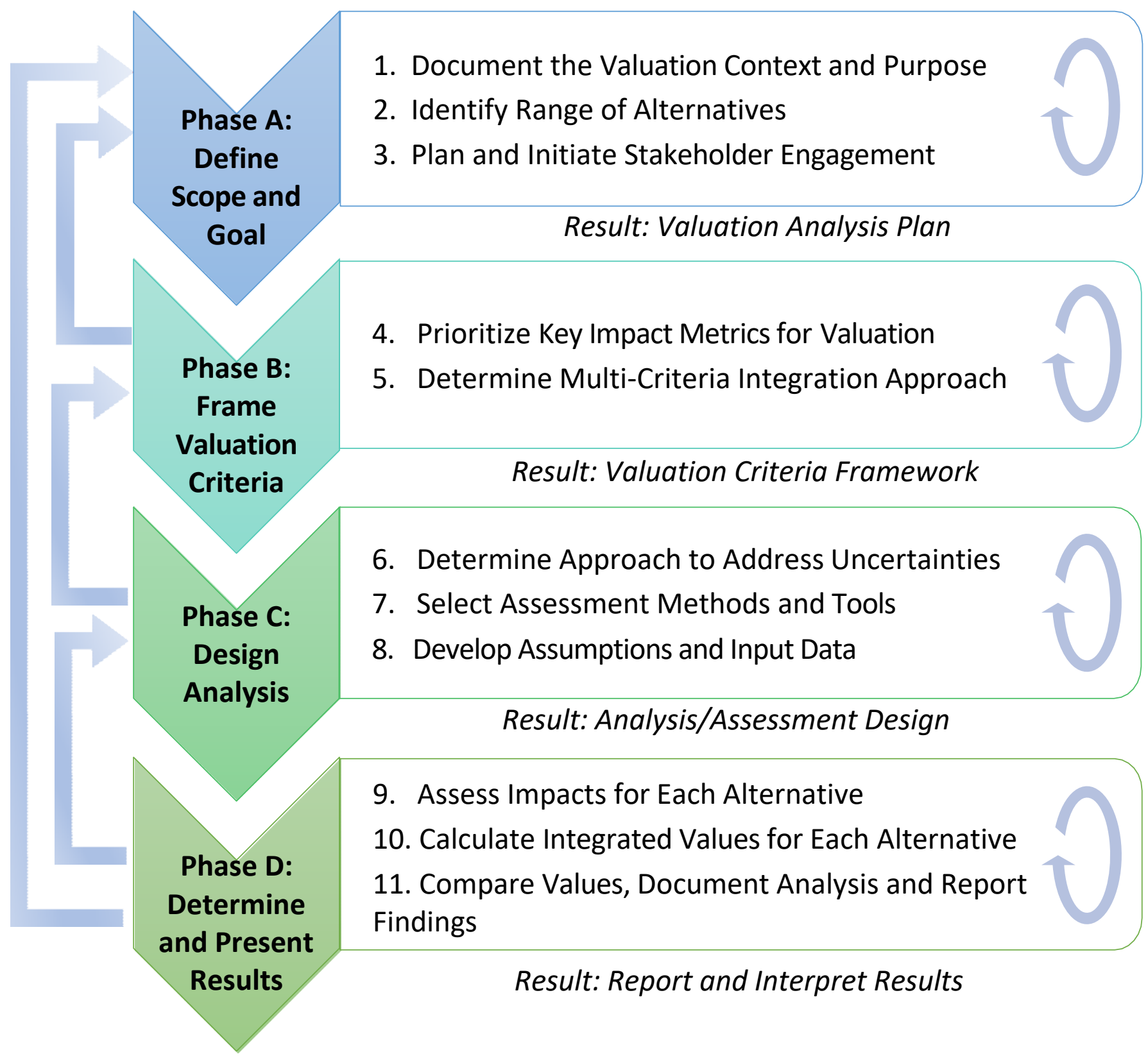





\section{PHASE A: DEFINE SCOPE AND GOAL}

\section{J.1 Step 1. Define the Valuation Context and Purpose}

Objectives: To define the purpose and scope of the valuation study and to develop a study plan

Primary purpose (succinct articulation of the valuation's primary goal, including
whose perspective is primary, the type of decision to be made, and the geographic area
and time frame being considered)

High-level specification of overall process to be followed

\section{Preliminary list of stakeholders to be involved}

Initial draft of the study plan (including time frame, budget, milestones, and evaluation teams)

Identify related studies (that can inform the selection of metrics and methods)

\section{Questions to consider}

- What valuation questions must or should be answered?

- What aspects of the power system should be included-or excluded?

- What are the physical boundaries and temporal ranges?

(Time frames may be short or long, depending on Step 3 stakeholder preferences and Step 4 performance impact metrics of most interest.)

\section{Notes}

- Stakeholders may be involved in different ways in Step 1.

- Broadly identify what constitutes "success." (Although metric selection is the focus of Step 4, this preliminary identification can help align the purpose and ultimate metrics.)

- Phase A steps are closely interrelated, and govern all subsequent Phases.

Create a "Valuation Analysis Plan" that documents choices and rationale 


\section{J.2 Step 2. Identify the Range of Alternatives}

Objective: To identify and select which alternatives to analyze and compare

Specify baseline or business-as-usual case (for comparison against alternatives)

Alternatives to consider in valuation (and the boundaries on those alternatives)
Questions to consider

- Are the alternatives

feasible?

- Why consider these alternatives (as opposed to other possibilities)?

\section{Notes}

- Budget constraints may limit the number of alternatives that can be considered.

- This step begins to specify bases for comparison among alternatives.

- Stakeholder engagement can play an important role in identifying and selecting among alternatives.

Document choices and rationale 


\section{J.3 Step 3. Plan and Initiate Stakeholder Engagement}

Objectives: To identify the stakeholders to be involved in the valuation study, define their roles, and develop a stakeholder engagement plan

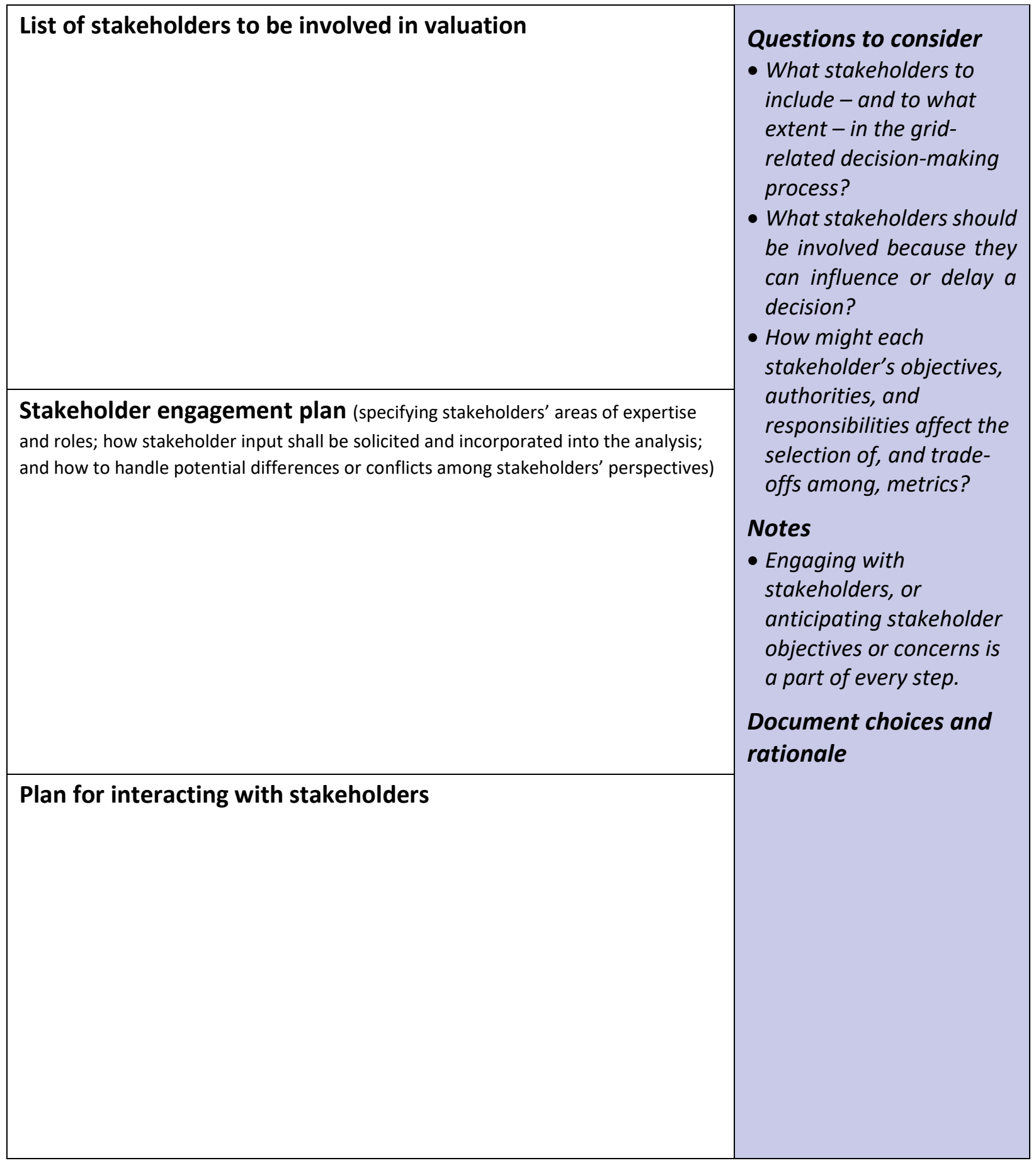




\section{PHASE B: FRAME VALUATION CRITERIA}

\section{J.4 Step 4. Identify Key Impact Metrics for Valuation}

Objectives: To (a) identify all the performance characteristics or metrics that will be used to characterize the outcome of choosing an alternative; (b) prioritize those metrics according to how they influence the valuation of alternatives; and (c) specify how higher-level or composite metrics (e.g., reliability, environmental quality, job creation) can be expressed in terms of basic metrics that are estimated or calculated by specific tools, models, or methods

Comprehensive list of metrics that could be used to categorize the attributes to be valued in the study

Prioritize the metrics that can be calculated within the scope, schedule and budget of the valuation study

Method to calculate or estimate each metric (e.g., data source, model or analysis output, combinations of other metrics)
Questions to consider

- What metrics must be calculated to achieve the purpose of the study?

- What metrics should be calculated, given resource constraints?

- How will you define/ specify/calculate metrics that could encompass many factors (e.g., "reliability" or "environmental quality")?

- Will you include non-gridrelated metrics (e.g., economic health of region)?

\section{Notes}

- Metrics are the "mortar" for the Valuation Framework, so this Step is influenced by-and influences-nearly every other Step.

- Metrics should align with the purpose of the analysis and the desired outcomes-what constitutes "success" (from Step 1).

Document choices and rationale 


\section{J.5 Step 5. Determine Multi-Criteria Integration Approach}

Objective: To develop a method to assign comparative values to alternatives characterized by disparate metrics

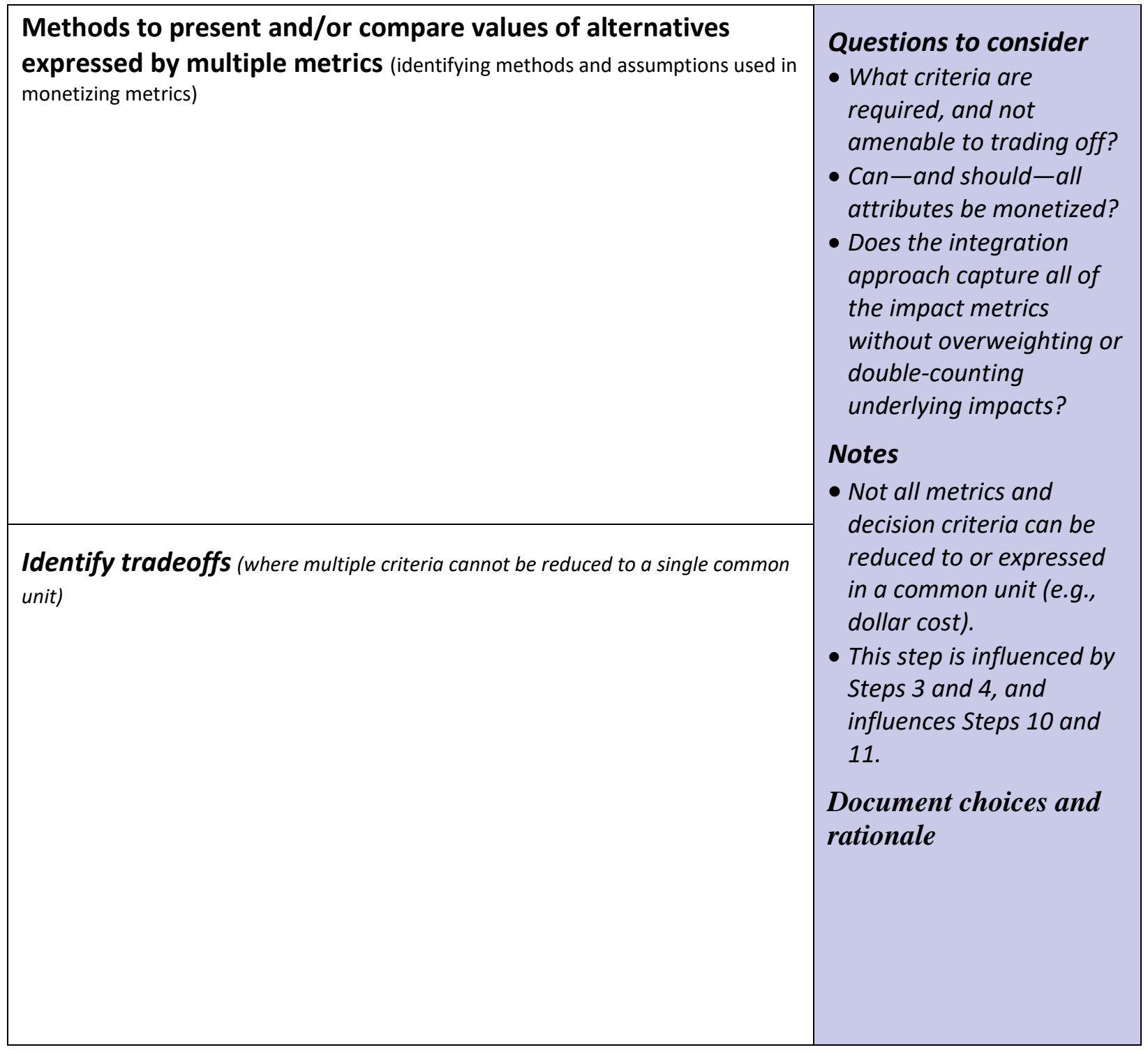




\section{PHASE C: DESIGN ANALYSIS}

\section{J.6 Step 6. Determine Approach to Address Uncertainties}

Objectives: To identify the unknowns and uncertainties inherent in forecasting, modeling, and valuing; to determine and document how to deal with these uncertainties (or estimates)

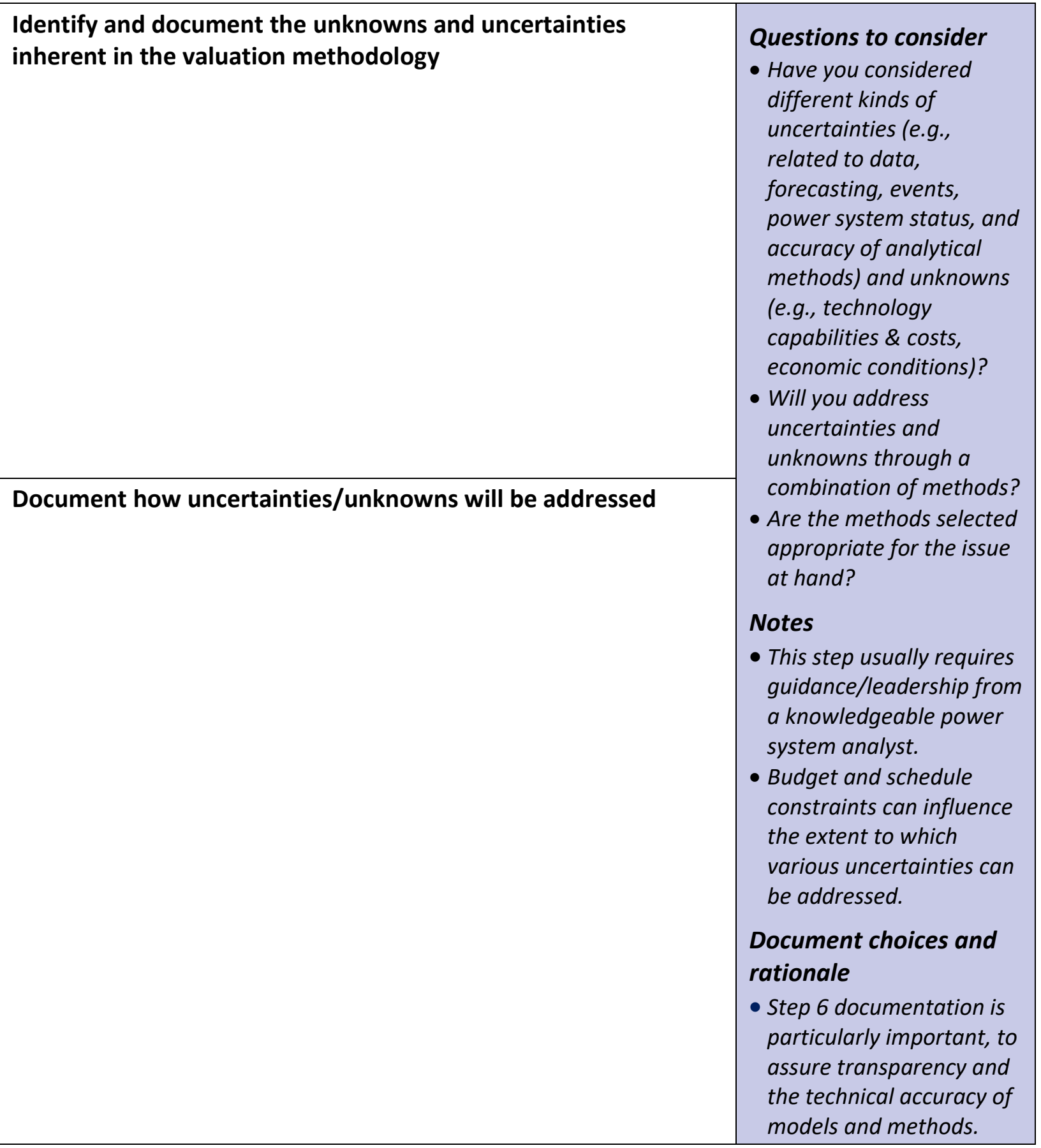




\section{J.7 Step 7. Select Assessment Methods and Tools}

Objectives: To select appropriate analysis methods, tools, and models consistent with the information needs previously identified and feasible within the budget and schedule set for the study

Calculation approach (data flows, methods, tools, calculations, etc. to calculate the performance metrics and impacts prioritized in Step 4)

\section{Questions to consider}

- Do analysis methods and tools accurately calculate decision-relevant metrics and impacts?

- What analyses need to be simplified due to budget and/or time constraints?

\section{Notes}

- The scope of the study and the information required to choose among alternatives (metrics) inform which analysis methods and tools are suitable.

- This step is influenced by Steps 1, 2, 4, 5, and 6.

Data needed for the calculation methods
Document choices and rationale 


\section{J.8 Step 8. Develop Assumptions and Input Data}

Objective: To identify and document all sources of data and simplifications or assumptions made during the analysis

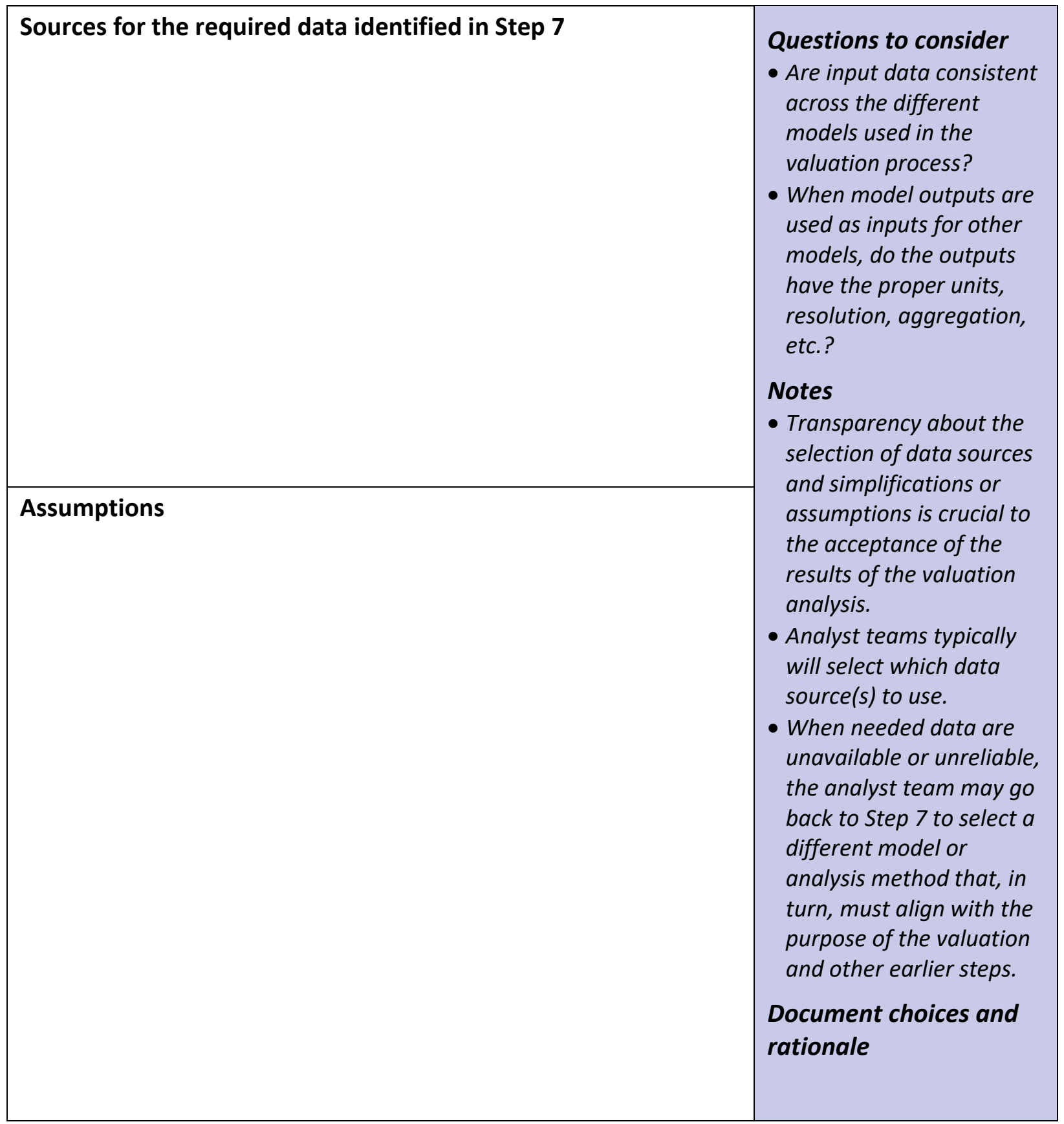




\section{PHASE D: DETERMINE AND PRESENT RESULTS}

\section{J.9 Step 9. Assess Impacts for Each Alternative}

Objectives: To exercise the analysis methodology and calculate the impacts/metrics of each alternative

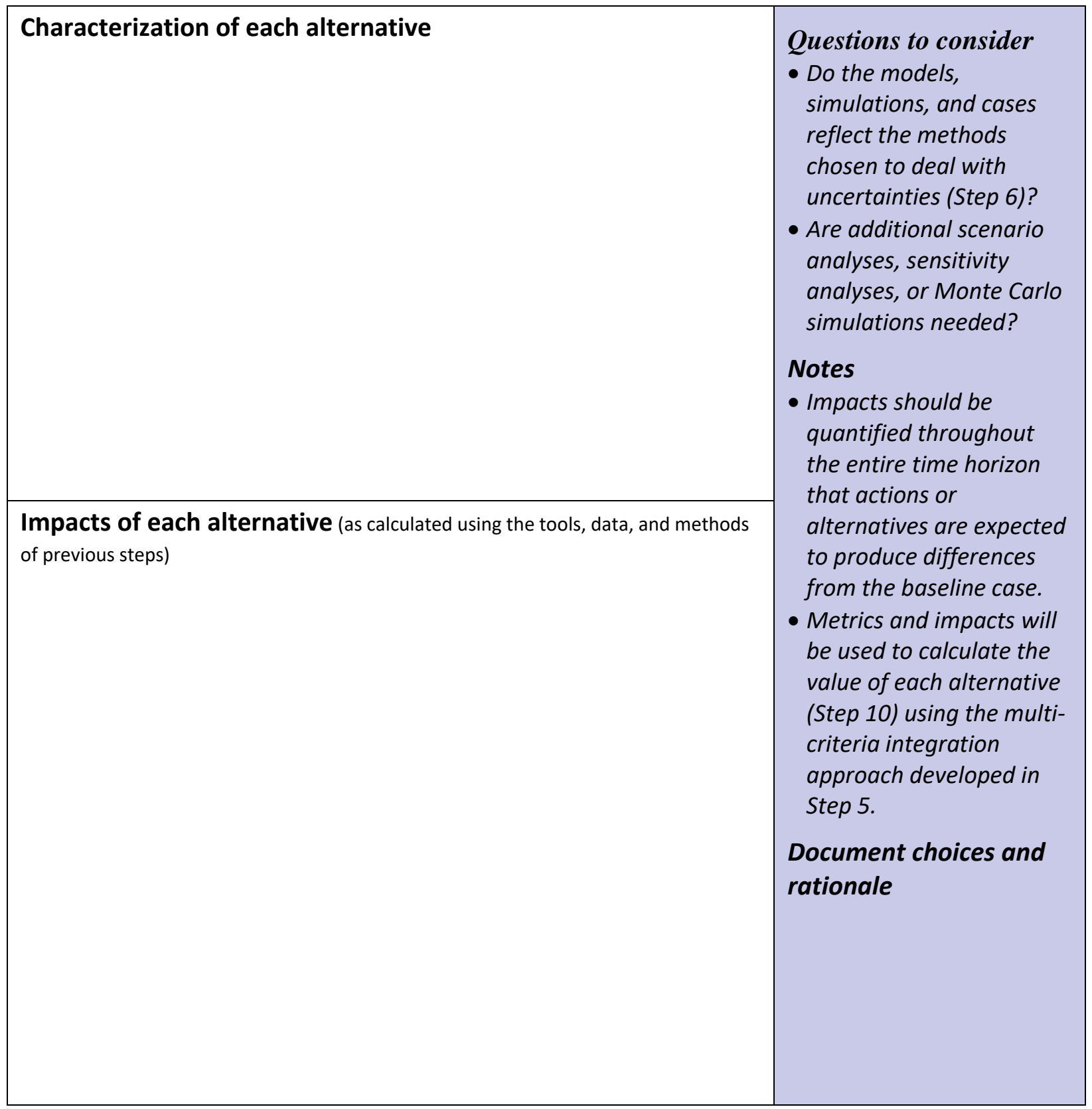




\section{J.10 Step 10. Calculate Integrated Values for Each Alternative}

Objective: To present the metrics and impacts that are used to assess the value for each alternative

\begin{tabular}{|c|c|}
\hline Value of each alternative (combining different types of expected impacts) & $\begin{array}{l}\text { Questions to consider } \\
\text { - Does the approach } \\
\text { chosen in Step } 5 \text { provide } \\
\text { sufficient methods for } \\
\text { integrating results? } \\
\text { - Are each alternative's } \\
\text { major characteristics } \\
\text { captured? } \\
\text { - Have the non- } \\
\text { quantifiable metrics been } \\
\text { included in the value } \\
\text { discussion? } \\
\text { - How are uncertainties } \\
\text { incorporated into these } \\
\text { results, or otherwise } \\
\text { communicated? } \\
\text { Notes } \\
\text { - Step } 10 \text { combines the } \\
\text { various impacts for each } \\
\text { alternative (Step 9) using } \\
\text { the multi-criteria } \\
\text { approach identified in } \\
\text { Step } 5 . \\
\text { Document choices and } \\
\text { rationale }\end{array}$ \\
\hline
\end{tabular}




\section{J.11 Step 11. Compare Values, Document Analysis, and Report Findings}

Objectives: To present the relative valuations of each alternative in a format that facilitates objective comparison by decision makers and other stakeholders; to prepare a final report and complete comprehensive documentation of the valuation study

Comparison of Alternatives (including quantitative and non-quantitative values, with uncertainties)
Final work products (Including any reports, briefings, presentations, datasets, and documentation of all methods, data, and assumptions)
Questions to consider

- Are tradeoffs among different impacts included in the comparison?

- Does the approach selected for communicating results provide sufficient information and insights for all stakeholders?

- Is the documentation produced in previous steps consistent and complete?

- How, and to whom, will the final report and documentation be disseminated?

\section{Notes}

- Step 11 is the culmination of all preceding steps.

Document choices and rationale

- The final work products should provide sufficient information for decision makers and sufficient detail for transparency and repeatability. 



\section{Appendix K: Evaluation of Process-Based Standards}

This is the cover page of a separate paper being published under the auspices of the GMLC 1.2.4 project.

\section{Evaluation of Process-Based Standards}

March 2019

Alan Cooke

Prepared for

the U.S. Department of Energy

under Contract DE-AC05-76RL01830

Pacific Northwest National Laboratory

Richland, Washington 99352 




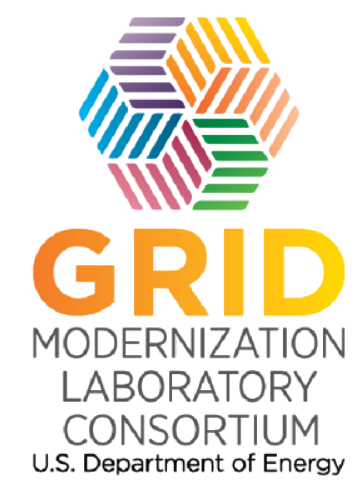

http://gridmodernization.labworks.org/ 